\title{
26. JURASSIC FORAMINIFERS FROM THE MAZAGAN PLATEAU, DEEP SEA DRILLING PROJECT SITE 547, LEG 79, OFF MOROCCO ${ }^{1}$
}

\author{
Wolfgang Riegraf and Hanspeter Luterbacher, Institut und Museum für Geologie und Paläontologie, \\ Universität Tübingen \\ and \\ R. Mark Leckie, Department of Geological Sciences, University of Colorado ${ }^{2}$
}

\begin{abstract}
The first marine incursion of the incipient North Atlantic Ocean is recorded in the uppermost Triassic to Lower Jurassic sequence of DSDP Site 547 off central Morocco. A lithologic change from continental red beds below to slope breccias and hemipelagic carbonates above indicates that a carbonate ramp was probably established by Sinemurian time along the Moroccan continental margin and that subsidence in the adjacent basin was rapid in the early phases of continental rift. Foraminifers recovered from the Liassic (Sinemurian-Pliensbachian) basinal deposits are diverse and well preserved. The faunas are compositionally similar to contemporaneous neritic assemblages of Europe and the Grand Banks of Newfoundland. The Middle Jurassic in Hole 547B is characterized by regressive deposits that are poor in foraminifers. The major Late Jurassic "Atlantic" transgression is again represented by basinal deposits consisting of limestone breccias and pelagic carbonates. Foraminifers recovered from this interval are transitional between Late Jurassic assemblages reported from deep-sea deposits in the North Atlantic and typical Late Jurassic neritic assemblages of Europe. The Late Jurassic assemblages of Hole 547B are primarily dominated by nodosariids and spirillinids with moderate abundances of simple arenaceous forms. Nonreticulate epistominids occur very rarely in the Upper Jurassic of Hole 547B. It is tentatively suggested that these represent upper bathyal assemblages.
\end{abstract}

\section{INTRODUCTION}

An undeformed sequence of Jurassic carbonates and carbonate breccias with lesser amounts of interbedded shales was drilled at Site 547 from the area of the distal continental margin off Morocco (Fig. 1). The Jurassic section at Hole 547B is relatively thin $(154 \mathrm{~m})$ and contains stratigraphic gaps, particularly between the Lias (Lower Jurassic) and Dogger (Middle Jurassic). Although this part of the Northwest African continental margin was essentially sediment-starved during the Jurassic, the vertical lithologic changes compare favorably with deepwater Tethyan sections from around the Mediterranean (Bernoulli and Kälin, this volume) and the central North Atlantic (Jansa et al., this volume). Transgressive/regressive phases inferred from the stratigraphic record of Site 547 generally parallel those interpreted from Moroccan epicontinental sections (Ager, 1974; Jansa and Wiedmann, 1982).

The Jurassic carbonate section of Site 547 (Unit VI) overlies (?)Rhaetian-Hettangian (uppermost TriassicLower Jurassic) continental red beds (Fenton, this volume). This site records the first marine incursion of the incipient central North Atlantic Ocean. The underlying continental deposits change rapidly upward to hemipelagic and mass-flow deposits of the slope environment. The Lower Jurassic (Core 547B-24 to Section 547B-14-2)

\footnotetext{
${ }^{1}$ Hinz, K., Winterer, E. L., et al., Init. Repts. DSDP, 79: Washington (U.S. Govt. Printing Office).

Addresses: (Riegraf, Luterbacher) Institut und Museum für Geologie und Palăontologie, Universităt Tübingen, Sigwartstrasse 10, D-7400 Tübingen 1, Federal Republic of Germany; (Leckie, present address) Department of Geology and Geography, University of Massachusetts, Amherst, MA 01003.
}

consists of limestone breccias overlain by redeposited(?) nodular limestones with minor black shales (Fig. 2). By Sinemurian time a carbonate ramp was probably already established along the adjacent Moroccan margin and was a source of the redeposited sediment.

Section 547B-14-2 through Sample 547B-11,CC contain limestone breccias. The core-catcher sample from Core 11 contains a rich foraminiferal fauna indicating a late Pliensbachian age, but a Middle Jurassic age is suggested from lithostratigraphic considerations (Steiger and Jansa, this volume; ?regressive Bajocian, Jansa et al., this volume). The Middle and Upper Jurassic section of Hole 547B consists of hardground cycles in Core 11 (Steiger and Jansa, this volume; Jansa et al., this volume) followed by limestone breccias and pelagic carbonates with intervals of turbidites in Core 547B-10 through Section 547B-6-2.

For detailed lithologic descriptions and interpretations of the upper Triassic-Jurassic sequence, we refer the reader to the site chapter, to chapters by Jansa et al., Bernoulli and Kälin, Steiger and Jansa, to the Leg 79 synthesis by Hinz and Winterer (this volume) and to Gradstein et al., 1975.

In this contribution we describe Jurassic foraminifers and other associated microbiota found in washed residues from Hole 547B. Figure 3 summarizes the distribution of the various foraminiferal superfamilies through the section. Figure 4 shows the distribution of the foraminiferal species recorded at Site 547B and their relative abundances, and Figure 5 the distribution and relative abundances of other microbiota encountered. For detailed biostratigraphic discussions of other fossil groups, the reader is referred to chapters by Azéma and Jaffrezo 

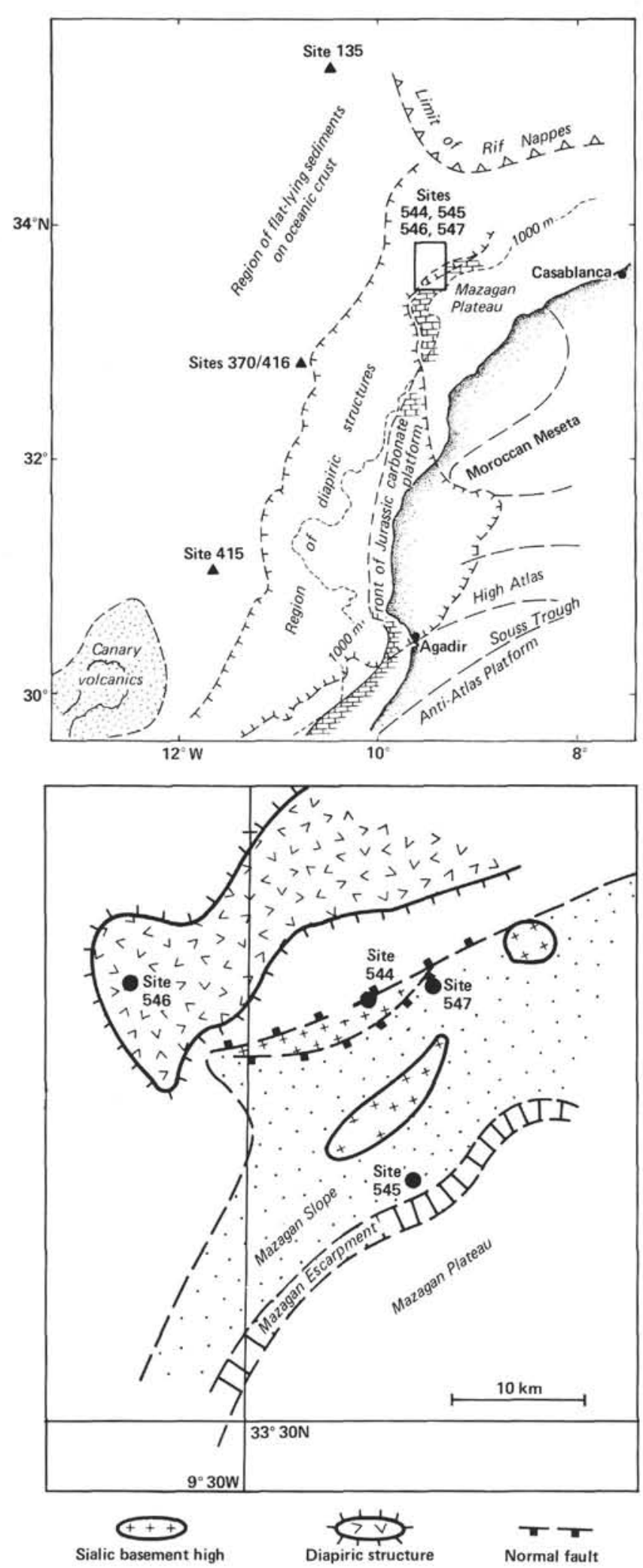

Figure 1. Regional setting of the Mazagan Plateau and sites drilled during Leg 79.

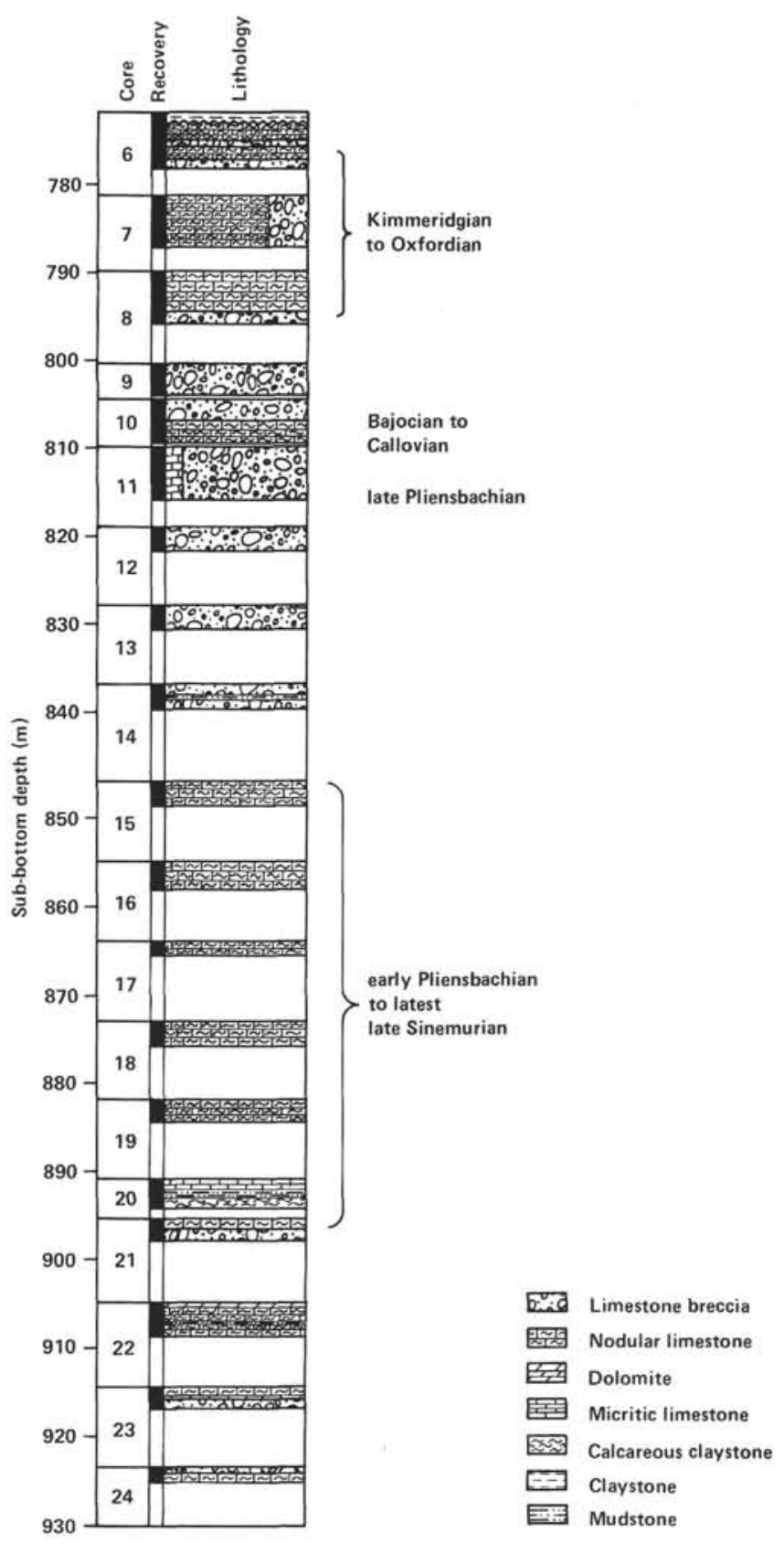

Figure 2. Detail of the Jurassic section drilled at Site 547 and summary of foraminiferal age assignments for Hole 547B (after Bernoulli and Kälin, this volume).

(calpionellids); Bate et al. (ostracodes); Fenton (palynology); Kälin and Bernoulli (Schizosphaerella); Renz (ammonites/aptychi); and Wiegand (nannofossils).

The taxonomy of Jurassic foraminifers and their stratigraphic significance are still very much open to contradictory interpretations. Some authors (e.g., Barteinstein and Brand, 1937) attribute a wide range of intraspecific variability to some groups of Jurassic foraminifers, especially to the representatives of such genera as Nodosaria, Dentalina, Lenticulina, Pseudonodosaria, Lingulina, and others. We may be accused of doing the contrary. However, we hope that the illustrations of the faunas will allow readers to reach their own conclusions on tax- 


\begin{tabular}{|c|c|c|c|c|c|c|c|c|c|c|c|}
\hline 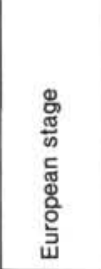 & $\begin{array}{l}\text { Core-Section } \\
\text { (interval in cm) }\end{array}$ & 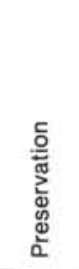 & $\begin{array}{c}\text { Assemblage } \\
\text { dominated by }\end{array}$ & 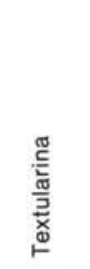 & 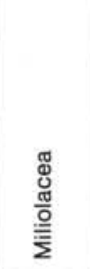 & 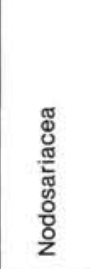 & 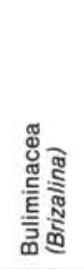 & 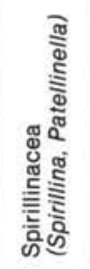 & 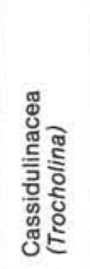 & 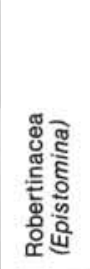 & 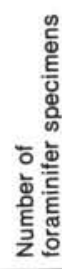 \\
\hline \multirow{6}{*}{ 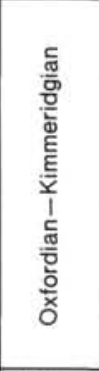 } & $6-4,49-51$ & $\begin{array}{l}\text { Very } \\
\text { bad }\end{array}$ & Nodosariids & $\stackrel{9}{(13 \%)}$ & \multirow[t]{2}{*}{$1(1 \%)$} & $\begin{array}{c}47 \\
(68 \%)\end{array}$ & \multirow{23}{*}{$2(1 \%)$} & $\begin{array}{c}11 \\
(16 \%)\end{array}$ & \multirow{23}{*}{$1(1 \%)$} & $1(1 \%)$ & 69 \\
\hline & $7-2,35-36$ & Bad & Spirillinids & $\begin{array}{c}26 \\
(14 \%)\end{array}$ & & $\begin{array}{c}14 \\
(8 \%)\end{array}$ & & $\begin{array}{c}142 \\
(78 \%)\end{array}$ & & & 182 \\
\hline & $7-4,135-137$ & Bad & $\begin{array}{l}\text { Spirillinids, } \\
\text { Nodosariids }\end{array}$ & $\begin{array}{c}16 \\
(9 \%)\end{array}$ & $9(5 \%)$ & $\begin{array}{c}76 \\
(41 \%)\end{array}$ & & $\begin{array}{c}80 \\
(43 \%)\end{array}$ & & $5(3 \%)$ & 186 \\
\hline & $7 . \mathrm{CC}$ & Bad & $\begin{array}{l}\text { Nodosariids } \\
\text { (spirillinids) }\end{array}$ & $\begin{array}{c}37 \\
(11 \%)\end{array}$ & $2(1 \%)$ & $\begin{array}{c}175 \\
(53 \%)\end{array}$ & & $\begin{array}{c}118 \\
(35 \%)\end{array}$ & & $1(1 \%)$ & 333 \\
\hline & $8-1,4-6$ & Bad & $\begin{array}{l}\text { Arenaceous } \\
\text { (nodosariids) }\end{array}$ & $\begin{array}{c}74 \\
(63 \%)\end{array}$ & & $\begin{array}{c}42 \\
(35 \%)\end{array}$ & & $2(2 \%)$ & & & 118 \\
\hline & $8-1,7-9$ & Bad & (Nodosariids) & $1(8 \%)$ & & $\begin{array}{c}9 \\
(75 \%)\end{array}$ & & $\begin{array}{c}2 \\
(17 \%)\end{array}$ & & & 12 \\
\hline $\begin{array}{l}\text { Baj.- } \\
\text { Callov. }\end{array}$ & $10-3,125-128$ & Bad & Nodosariids & $1(1 \%)$ & $1(1 \%)$ & $\begin{array}{c}125 \\
(82 \%)\end{array}$ & & $\begin{array}{c}25 \\
(16 \%)\end{array}$ & & & 152 \\
\hline ?Dogger & $11-1,147-150$ & & & & & & & & & & \\
\hline $\begin{array}{l}\text { late } \\
\text { Pliens.? }\end{array}$ & $11, \mathrm{CC}$ & Bad & Nodosariids & $7(2 \%)$ & $7(2 \%)$ & $\begin{array}{l}254 \\
(90 \%)\end{array}$ & & $\begin{array}{l}11 \\
(4 \%)\end{array}$ & & & 282 \\
\hline \multirow{9}{*}{ 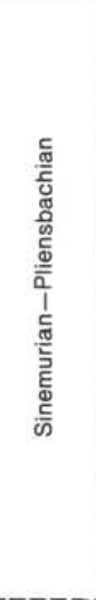 } & $14, \mathrm{CC}$ & & & & & & & & & & \\
\hline & $15-1,0-4$ & Good & Nodosariids & $1(2 \%)$ & & $\begin{array}{c}39 \\
(93 \%)\end{array}$ & & $2(5 \%)$ & & & 43 \\
\hline & $17, \mathrm{CC}$ & Good & Nodosariids & & & $\begin{array}{l}50 \\
(98 \%)\end{array}$ & & & & & 51 \\
\hline & $18-1,22-24$ & Good & Nodosariids & & & $\begin{array}{c}54 \\
(96 \%)\end{array}$ & & $2(4 \%)$ & & & 56 \\
\hline & $18-2,5-7$ & $\begin{array}{l}\text { Very } \\
\text { good }\end{array}$ & Nodosariids & $2(1 \%)$ & & $\begin{array}{c}225 \\
(97 \%)\end{array}$ & & $4(2 \%)$ & & & 233 \\
\hline & $20-1,126-129$ & $\begin{array}{l}\text { Very } \\
\text { good }\end{array}$ & Nodosariids & $\begin{array}{c}20 \\
(8 \%)\end{array}$ & & $\begin{array}{l}214 \\
(92 \%)\end{array}$ & & & & & 234 \\
\hline & $\begin{array}{l}20-2,59-61 \\
21, \mathrm{CC}\end{array}$ & $\begin{array}{l}\text { Very } \\
\text { good }\end{array}$ & $\begin{array}{l}\text { Nodosariids } \\
\text { (arenaceous) }\end{array}$ & $\begin{array}{c}34 \\
(40 \%)\end{array}$ & & $\begin{array}{c}50 \\
(59 \%)\end{array}$ & & $1(1 \%)$ & & & 85 \\
\hline & $22-1,95-98$ & $\begin{array}{l}\text { Very } \\
\text { good }\end{array}$ & (Nodosariids) & & & $\begin{array}{c}22 \\
(100 \%)\end{array}$ & & & & & 22 \\
\hline & $22-2,55-57$ & $\begin{array}{l}\text { Very } \\
\text { good }\end{array}$ & $\begin{array}{c}\text { Nodosariids } \\
\text { (arenac., milio.) }\end{array}$ & $\begin{array}{c}35 \\
(20 \%)\end{array}$ & $\begin{array}{c}36 \\
(20 \%)\end{array}$ & $\begin{array}{c}105 \\
(60 \%)\end{array}$ & & & & & 176 \\
\hline \multirow{5}{*}{ 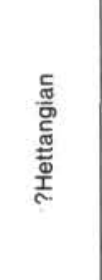 } & $24-1,139-141$ & Bad & (only Ostracodes) & & $? 1$ & & & & & & $? 1$ \\
\hline & $24, \mathrm{CC}, 10-12$ & Good & (only Ostracodes) & & & & & & & & \\
\hline & $24, \mathrm{CC}$ & Bad & (only Ostracodes) & & & & & & & & \\
\hline & $25, \mathrm{CC}$ & & & & & & & & & & \\
\hline & $26, \mathrm{CC}$ & & & & & & & & & & \\
\hline
\end{tabular}

Figure 3. Foraminiferal assemblages recovered from Hole 547B. Note the good to excellent preservation of the Liassic faunas with nodosariids typically representing $>90 \%$ of the assemblages. The Late Jurassic assemblages are characterized by poor preservation; spirillinids and nodosariids dominate.

onomy as well as stratigraphic and paleoenvironmental significance. The senior authors (W.R. and H.L.) are responsible for the detailed taxonomy and distribution tables. R.M.L. is responsible for the paleoenvironmental assessment of the Jurassic assemblages.

\section{DISCUSSION OF THE MICROFAUNAS IN HOLE 547B}

Core 24: The washed residues from Core 24 contain only ostracodes and several, mostly indeterminate, mili- olids and primitive arenaceous foraminifers which cannot be used for an age determination.

Core 23: No samples were examined from Core 23.

Core 22: The oldest well-preserved microfaunas are from Core 22. In addition to echinoid spines and holothurian remains, the two samples from this core contain well-preserved foraminiferal assemblages composed of primitive arenaceous foraminifers, miliolids and nodosariids. The assemblages are attributed a latest late Sinemurian to early Pliensbachian age since they are in good 


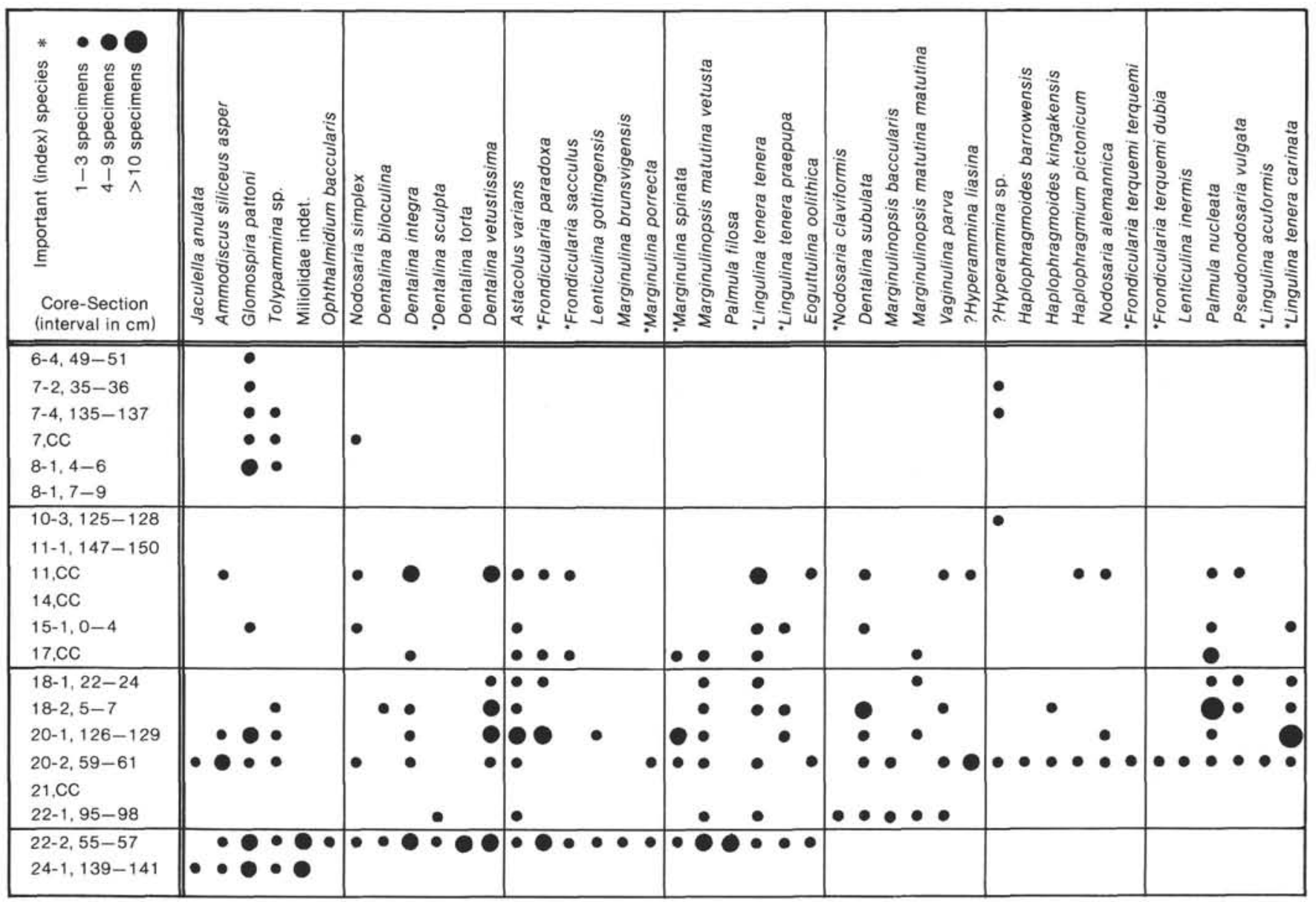

Note: Samples $5478-24, \mathrm{CC}(10-12 \mathrm{~cm}), 547 \mathrm{~B}-25, \mathrm{CC}$, and $547 \mathrm{~B}-26, \mathrm{CC}$ barren of benthic foraminifers

Figure 4. Distribution and relative abundances of the benthic foraminifers recovered from the Jurassic section of Hole 547B (identifications by W. R. and H. L.).

agreement with microfaunas of this age from central and western Europe.

Core 21: The core-catcher sample from Core 21 is barren.

Core 20: The two samples available from Core 20 contain a well-preserved and rich microfauna with a diversified foraminiferal assemblage dominated by nodosariids. The assemblages are similar to those from ageequivalent shale beds of western Europe, but their size is smaller. The early Pliensbachian age attributed to these assemblages is based on the presence of Frondicularia paradoxa, $F$. terquemi terquemi, $F$. terquemi dubia, $F$. squamosa, Lingulina tenera tenera, L. tenera praepupa, L. tenera carinata, and Marginulina spinata (Copestake and Johnson, in press).

Core 19: No samples available.

Core 18: The sample from $547 \mathrm{~B}-18-2,5-7 \mathrm{~cm}$ is similar to Core 20 in composition and preservation. The assemblage from Section 547B-18-1 is considerably lower in diversity, but the composition is essentially the same as in Core 20.

Core 17: The poor but well-preserved autochthonous foraminiferal assemblage is similar in composition to those of Cores 18 and 20.

Core 16: No samples available.
Core 15: The only sample examined from this core is rather poor. It contains representatives of the same nodosariid species already recorded from Cores 18 and 20 and is probably still of early Pliensbachian age.

Core 14: The core-catcher sample is barren.

Core 13 and 12: No samples available.

Core 11: Only the core-catcher sample yielded a fairly rich but poorly preserved foraminiferal assemblage. It differs from the underlying Liassic assemblages in its poor preservation and in being dominated by ostracodes that indicate a Pliensbachian age; most of these are not recorded from the older samples. Also, the foraminiferal fauna contains some species which are not recorded in the underlying samples (e.g., Brizalina liasica, Dentalina gyrosa, Palmula primaria, Lingulina dentaliniformis, and L. gottingensis). The assemblage is still Early Jurassic in age, probably late Pliensbachian since there are no diagnostic Toarcian elements present. Noteworthy however is the dominance of Eoguttulina liassica and Ophthalmidium liasicum in 547B-11,CC. Exton and Gradstein (in press) have noted that these two species sometimes dominate the foraminiferal assemblage of the Portuguese early Toarcian (Falciferum and Bifrons zones).

The faunal change probably reflects the accompanying vertical increase in calcium carbonate content of the 


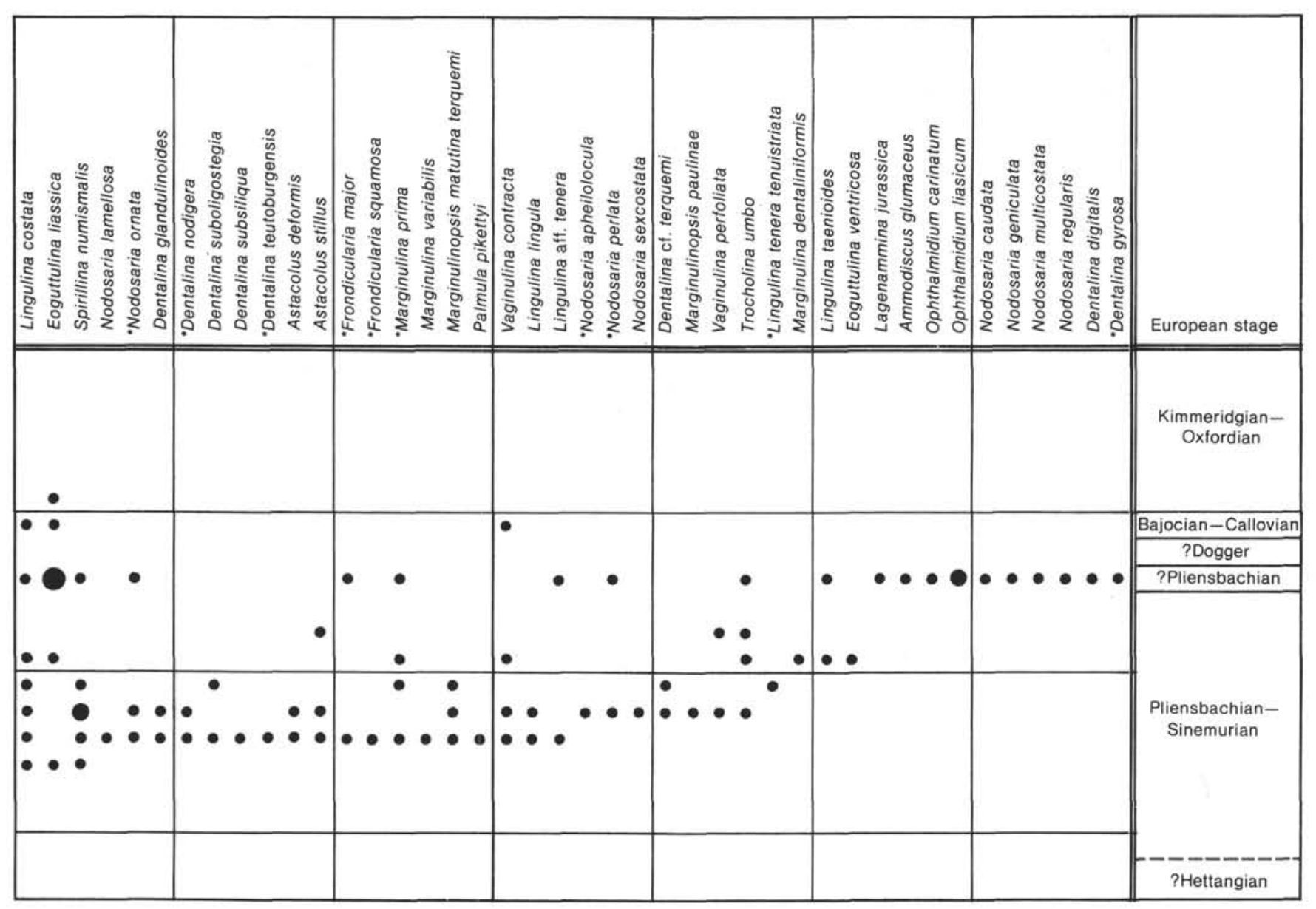

Figure 4. (Continued).

strata. In southwest Germany, microfaunas from shales poor in $\mathrm{CaCO}_{3}$ are generally rich in Dentalina, Frondicularia, Astacolus, Marginulina, ornamented Linguli$n a$, and species of Diademopsis (echinoid), whereas those from calcareous marls are generally dominated by ostracodes and smooth species of Lingulina comparable to those from Core 11. These vertical changes may indicate that the assemblage thrived in a shallower carbonaterich environment.

Core 10: Only one sample was examined from Core 10. It contains a fairly rich, but poorly preserved foraminiferal assemblage. The presence of Citharina colliezi and $C$. inconstans together with $L$. nodosaria point to a Bajocian to Callovian age. Ostracodes also indicate a Middle Jurassic age for this interval (Bajocian-Bathonian; Bate and Lord, this volume). Radiolarians are common.

Core 9: No samples available.

Core 8: The two poorly preserved foraminiferal assemblages examined from Core 8 are composed mainly of agglutinating species (Glomospira sp., Haplophragmoides globigerinoides, and Bigenerina arcuata) and a few nodosariids (including Lenticulina quenstedti). This assemblage is comparable to those which are described from DSDP Legs 11, 41, 44, and 76, and which are considered to be of Oxfordian to Kimmeridgian age.
Core 7: The three samples from Core 7 are rich in Spirillina spp. and in a form determined as "Patelinella" poddari, which needs further study to determine its taxonomic position. The stratigraphically important species are Ophthalmidium strumosum, L. quenstedti, and Lingulina franconica. Section 547B-7-2 includes strongly corroded tests of Lenticulina, which could be allochthonous. The foraminiferal assemblages are similar to those described from Oxfordian-Kimmeridgian deposits from Legs 11 and 41. Radiolarians are more frequent than in the sample from Section 547B-10-3.

Core 6: The single assemblage seen from Core 6 is rich, but its preservation is so bad that most forms are indeterminable. The general aspect indicates that it is not significantly younger than the assemblages from Core 7. Index species are absent.

\section{FORAMINIFERAL BIOSTRATIGRAPHY AND PALEOENVIRONMENTAL ANALYSIS}

\section{Lower Jurassic (Core 22 through Section 547B-14-2)}

The Lower Jurassic (upper Sinemurian-Pliensbachian) of Hole 547B correlates, in part, with the Involutina liassica Zone of the Grand Banks area (Gradstein, 1976), the Frondicularia terquemi Zone of the Lusitanian Basin, Portugal (Exton and Gradstein, in press) and the 


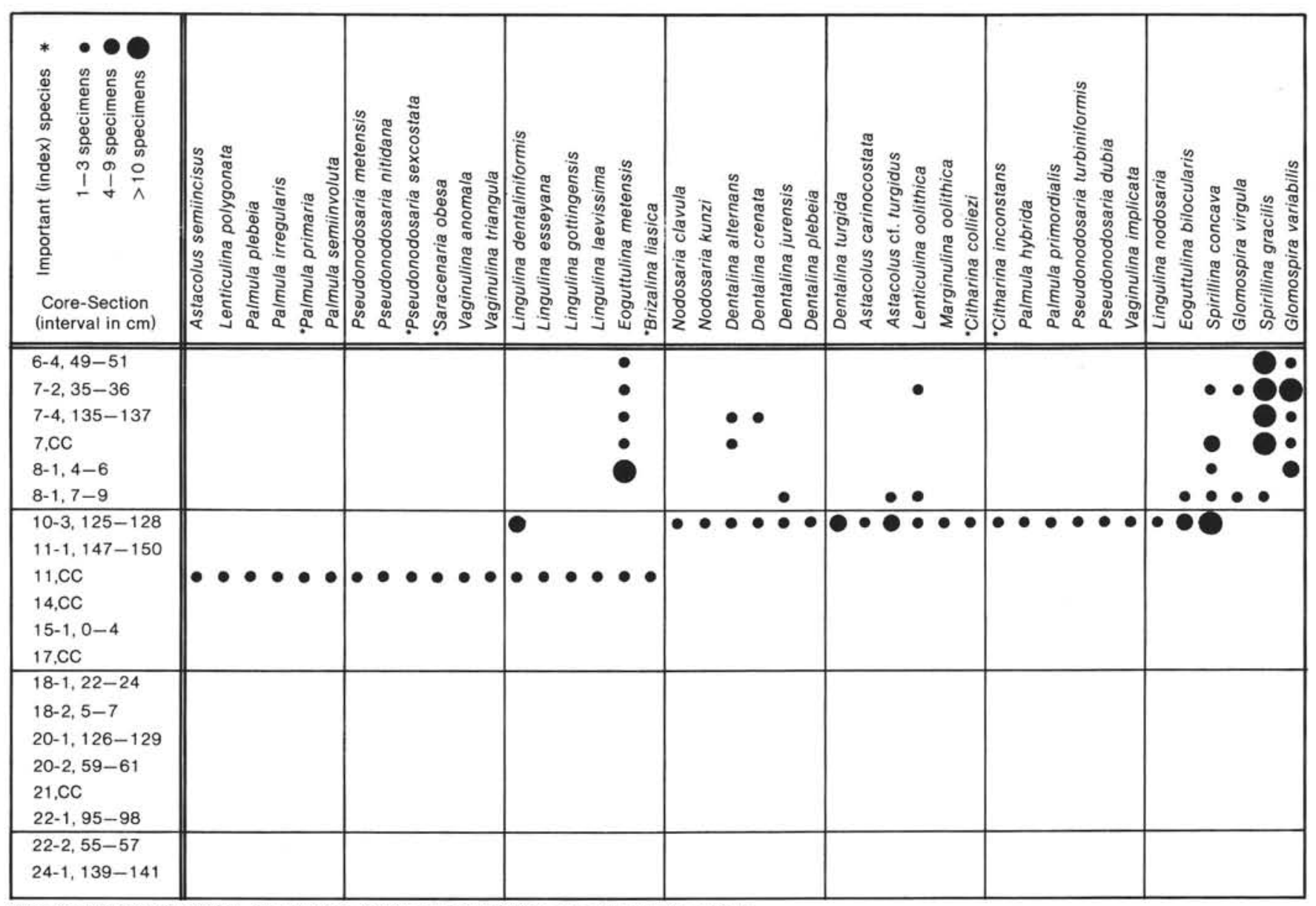

Note: Samples $547 \mathrm{~B}-24, \mathrm{CC}(10-12 \mathrm{~cm}), 547 \mathrm{~B}-25, \mathrm{CC}$, and 547B-26,CC barren of benthic foraminifers

Figure 4. (Continued).

Marginulina prima plexus interrupta Zone of Copestake and Johnson's comprehensive Early Jurassic foraminiferal zonation (in press). This correlation is based primarily on the presence of $M$. prima, F. terquemi, and Lingulina tenera in the late Sinemurian-Pliensbachian of the Grand Banks, Lusitanian Basin, and offshore Morocco. In addition, the association of $M$. spinata, Dentalina sculpta, Marginulinopsis matutina, and $F$. squamosa support a latest Sinemurian-early Pliensbachian age (Copestake, pers. comm., 1983).

The faunas from Core 22 through Section 547B-14-2 show close compositional similarities with their counterparts of Europe and the Grand Banks. Like their northern relatives, the assemblages of Hole 547B are dominated by nodosariids, but the specimens tend to be smaller than those of Europe. The assemblages of this interval are best classified as Type A-1 shelf assemblages of Gordon (1970). However, lithologic evidence indicates that the hemipelagic ?redeposited nodular limestones with minor, thin black shales represent basinal deposits near the base of the carbonate slope (Jansa et al., this volume). The well-preserved, diverse foraminiferal faunas from the rare clayey intervals suggest that these assemblages are truly autochthonous and flourished in this deeper marine environment. The Early Jurassic assemblages of Hole 547B may represent outer shelf/upper bathyal depths, but the homogeneous nature of Early
Jurassic foraminiferal faunas prevents paleobathymetric distinction from other "shelf" (Type A) assemblages.

\section{Lower or Middle Jurassic? (Sections 547B-14-2 to 547B-11,CC)}

Biostratigraphic control in this interval of sandy limestone breccias is limited. A poorly preserved but fairly rich foraminiferal fauna indicating a late Pliensbachian age was identified from Core 547B-11,CC. Pliensbachian ostracodes (Bate et al., this volume) and calcareous nannofossils (Wiegand, this volume) were also recovered from this interval. However, lithostratigraphic considerations suggest a Middle Jurassic age for the limestone breccias (Jansa et al., this volume; Steiger and Jansa, this volume).

The foraminiferal assemblage contrasts markedly with the excellent preservation observed in the late Sinemurian-Pliensbachian assemblages below. The lithology and fauna from 547B-11,CC indicate a different depositional environment than the underlying Lower Jurassic strata. The abundance of Ophthalmidium spp. suggests a shallow environment (Shipp and Murray, 1981).

Three possibilities exist to explain the occurrence of the limestone breccias. First, they may represent slope talus generated by regional shallowing or tectonic movements (subsidence) in the mid to late Early Jurassic. In this case the foraminiferal assemblage of Sample 547B- 


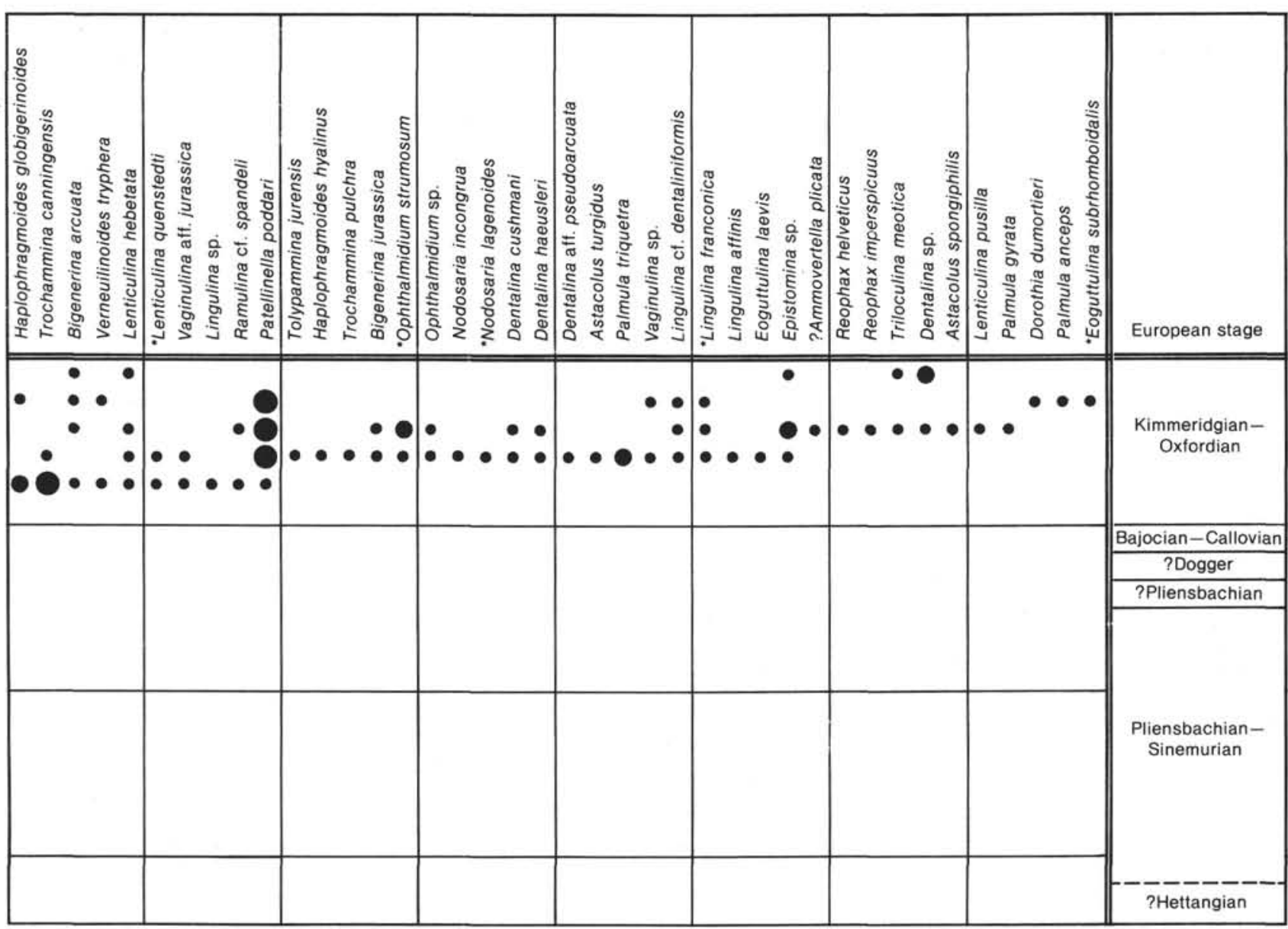

Figure 4. (Continued).

$11, \mathrm{CC}$ could be autochthonous or penecontemporaneously reworked from shallower deposits and the poor preservation of the assemblage may have resulted from a harsh preservational environment, diagenetic effects, or transport. A second possibility is that the limestone breccias were shed into the young Moroccan offshore basin during the Toarcian (late Early Jurassic) when tectonic events affected the adjacent Moroccan Meseta (see discussion by Lancelot and Winterer, 1980). In this case, the slightly older, Pliensbachian neritic assemblage was redeposited downslope. A third explanation is that the limestone breccias were deposited during the major Middle Jurassic (Bajocian ?) regression recorded around the North Atlantic (Ager, 1974; Jansa and Wiedmann, 1982). Again, in this case, the fauna of Sample 547B-11,CC was recycled from older deposits, though from a different (shallower ?) facies than the Sinemurian-Pliensbachian assemblages. Because no Middle Jurassic taxa were recovered from this interval, the latter two explanations are doubtful interpretations.

\section{Middle and Upper Jurassic (Sections 547B-11-4 through 547B-6-2)}

Core 11 contains 12 hardground cycles which are interpreted by Jansa et al. (this volume) to represent Middle Jurassic transgressive deposits characterized by very low sedimentation rates. Our sample from the upper part of Core 11 is barren of microfauna.

The nodular limestones from Section 547B-10-3 yielded a fairly rich foraminiferal assemblage indicating a Bajocian to Callovian age. Correlation with a specific foraminiferal zone of the once contiguous Grand Banks area (Gradstein, 1976) cannot be established. The sample lacks such neritic(?) genera as Epistomina, Reinholdella, and Garantella, which are important elements of the Grand Banks Middle Jurassic assemblages.

No shales are present in the interval of limestone breccias in Sections 547B-10-2 through 547B-8-4.

The widespread Late Jurassic "Atlantic" transgression is recorded in Sections 547B-8-3 through 547B-6-2. The succession is characterized by pelagic bivalve-bearing limestones, Saccocoma-bearing limestones, and calpionellid-bearing limestones, respectively, with interbedded turbidite layers. This implies that the carbonate slope had become more stable and permitted deposition of pelagic facies. The succession of pelagic facies represents deposition from Oxfordian through Tithonian times (Bernoulli and Kälin, this volume). Seafloor spreading during the Middle and Late Jurassic and different depositional settings have rendered correlation between Site 547 and the Grand Banks area tenuous. The OxfordianKimmeridgian interval of the Grand Banks corresponds 


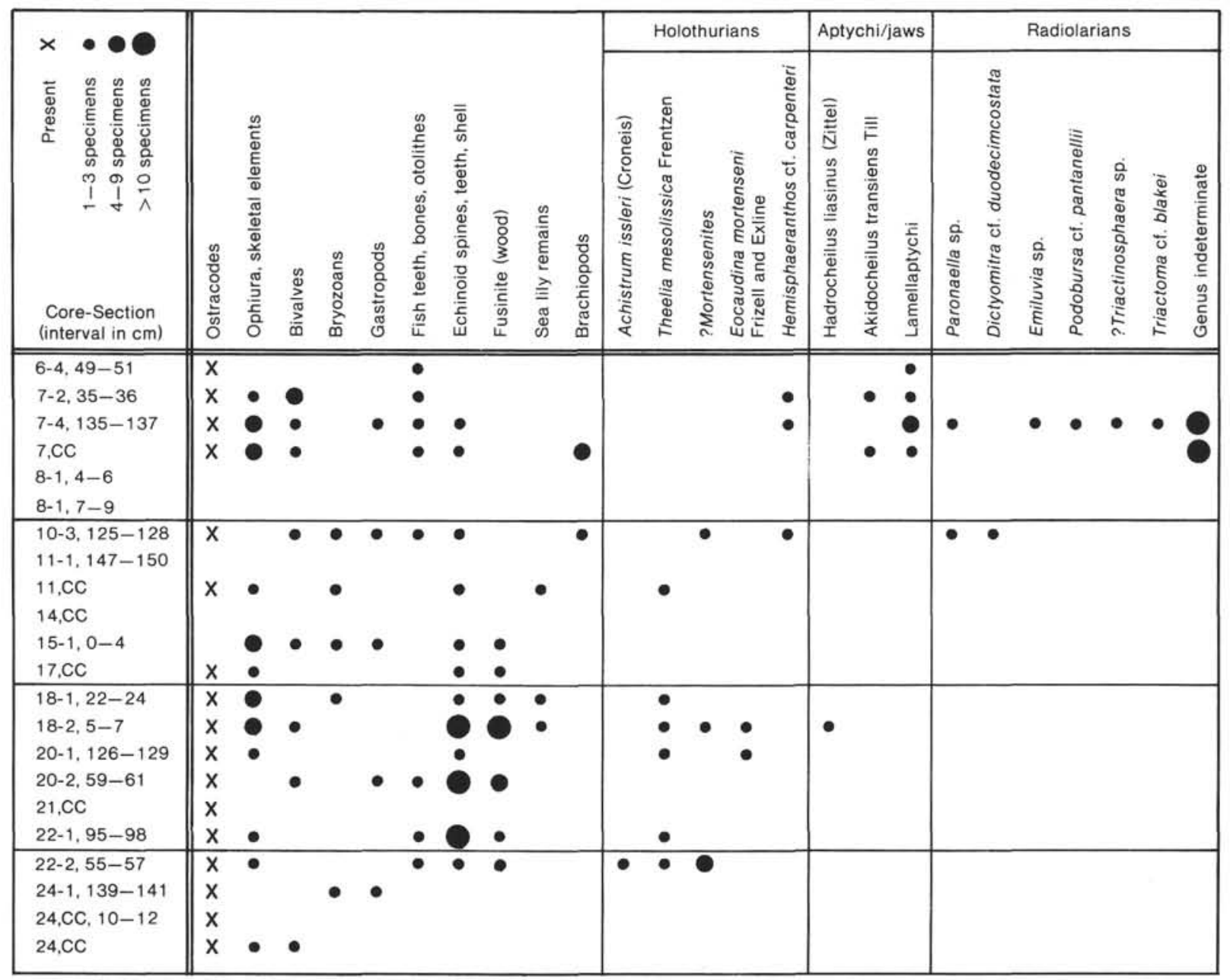

Note: $547 \mathrm{~B}-25, \mathrm{CC}$ and $547 \mathrm{~B}-26, \mathrm{CC}$ barren of such remains

Figure 5. Distribution and relative abundances of other microbiota recovered from the Jurassic section of Hole 547B (compiled by W. R. and H. L.). Ostracodes are treated separately by Bate et al. (this volume).

with the Epistomina mosquensis Zone of Gradstein (1976).

The Late Jurassic assemblages of Hole 547B are generally characterized by a codominance of nodosariids and spirillinids with moderate abundances of simple arenaceous forms (Fig. 4). Small, reticulate or smooth species of Epistomina, which are widely recorded in great abundances in certain intervals of Middle and Upper Jurassic neritic sequences (Gordon, 1970), are conspicuously lacking at Site 547. This may be an artifact of the poor preservation in this part of the section and the few shale samples that could be collected, or could be the result of environmental exclusion.

The faunas described from this part of the section are somewhat transitional in nature between Late Jurassic North Atlantic deep-sea assemblages (Luterbacher, 1972; Kuznetsova and Seibold, 1977; Gradstein, 1983) and "typical" neritic assemblages (Type A-1 shelf assemblages; Gordon, 1970). An upper bathyal environment is tentatively suggested for the Late Jurassic assemblages of Hole 547B. Although Jurassic spirillinids have been considered indicative of shallow-water conditions by some authors (e.g., Shipp and Murray, 1981), Luterbacher
(1972) and Kuznetsova and Seibold (1977) report a great abundance of "Spirillina" from bathyal deposits of the North Atlantic. Additional evidence in support of a bathyal environment for the Late Jurassic of Hole 547B is the lack of such predominantly neritic, open-shelf genera as Reinholdella, Conorboides, and Garantella. The epistominids that are present more closely resemble the deep-water, unornamented forms described by $\mathrm{Lu}$ terbacher (1972) and Gradstein (1983).

\section{TAXONOMIC REMARKS}

Bryozoans. (Pl. 3, Fig. 96.) Some forklike branches have been found in some samples.

Echinoids. (Pl. 3, Fig. 101.) Most of the Liassic spines and teeth belong to Diademopsis or related genera.

Ophiuroids. (Pl. 3, Figs. 103, 106, 107.) Most the Liassic remains are vertebrae of Ophiomusium. Figure 104 on Plate 3 seems also to be ophiuroid.

Holothurian sclerites. (PI. 3, Figs. 97-100, 102, 105.)

Achistrum issleri (Croneis in Croneis and McCormack, 1932, p. 128, pl. 18, figs. 50-51; Sinemurian and Pliensbachian (Rioult, 1961, table 1).

Eocaudina mortenseni Frizzell and Exline (1955, p. 88, pl. 3, fig. 10); Sinemurian of France (Rioult, 1961, table 1), southwest and north Germany. 
Theelia mesoliassica (Frentzen, 1964, p. 44, pl. 4, figs. 20-32); Sinemurian to lower Toarcian (southwest Germany). It has 6 to 12 spokes.

Hemisphaeranthos cf. carpenteri (Moore, 1873) (Frizzell and Exline, 1955 , p. 130 , pl. 7, fig. 16) has 15 spokes and internal teeth. Our illustrated specimen has 30 teeth; the holotype shows only 26.

Mortensenites? sp. are small, calcitic, irregular polygonal white plates with a dotted surface.

Cephalopods. (Pl. 1, Figs. 108-109.) In Upper Jurassic samples, lamellaptychi of perisphinctid ammonites are common.

Hadrocheilus liasinus (Zizzel, 1869), described as Rhynchoteuthis liasina Zittel (1869, p. 120, pl. 13, fig. 6) and as Hadrocheilus regularis Bessler, 1938 (p. 71, text.-fig. 2) occurs only at the base of the upper Pliensbachian (SW Germany, England, Italy). It probably belongs to the dibranchiate (coleoid) genus Chitinoteuthis Müller-Stoll, 1936, of the same stratigraphic age.

Akidocheilus transiens Till, 1907 (p. 637, pl. 13, fig. 26) is found in some Upper Jurassic samples. In length it reaches $8 \mathrm{~mm}$.

Foraminifera

Note that + identifies the species holotype

Hyperammina? liasina (Frentzen, 1941)

(P1. 4, Fig. 110)

+ 1941 Psammophax liasina Frentzen, p. 300, pl. 1, fig. 1 .

1961 Hyperammina odiosa Gerke and Sossipatrova in Gerke, p. 103, pl. 7, figs. 3-6.

Remarks. A very coarsely agglutinated species, which was originally described from the Pliensbachian.

\section{Hyperammina? sp. \\ (Pl. 4, Fig. 113)}

Remarks. Slender tubes, less coarsely agglutinated than Hyperammina? liasina (Frentzen). Possible fragments of Rhizammina Brady, 1879.

\section{Jaculella anulata Barbieri, 1964}

(Pl. 4, Figs. 111-112)

+1964 Jaculella anulata Barbieri, p. 744, pl. 56, fig. 1 .

1968 Jaculella liassica Brand. Welzel, p. 4, pl. 1, fig. 2.

Remarks. Differs from Jaculella liassica Brand, 1937, in possessing concentric thickenings and an irregular outline. Pliensbachian (Italy, Franconia).

\section{Lagenammina jurassica (Barnard, 1959)}

(Pl. 1, Fig. 7)

1936 Proteonina ampullacea (Brady). Franke, p. 13, pl. 1, fig. 7. 1937 Proteonina difflugiformis (Brady). Bartenstein and Brand, p. 128,

pl. 1A, fig. 1; pl. 1B, figs. 1-2; pl. 2A, fig. 1 ; pl. 2B, fig. 3 ; pl. 3,

fig. 1 ; pl. 4 , fig. 1 ; pl. 5 , fig. 1 ; pl. 8 , fig. 1 ; pl. 10, fig. 1 ; pl. 11 , fig. 1.

+1959 Proteonina jurassica Barnard, p. 134, pl. 11, figs. 6-8.

1968 Reophax difflugiformis (Brady). Welzel, p. 6, pl. 1, fig. 4.

Remarks. A widespread species, which ranges from Hettangian to the upper Middle Jurassic. It is finely to coarsely agglutinated and often compressed. The flask-shaped outline of this species is distinctive.

\section{Ammodiscus glumaceus Gerke and Sossipatrova, 1961}

$$
\text { (Pl. 1, Fig. 8) }
$$

+ 1961 Ammodiscus glumaceus Gerke and Sossipatrova in Gerke, p. 128 , pl. 12 , figs. $2-6$; pl. 13 , fig. 15 .

Remarks. A small Ammodiscus with fewer whorls and of more irregular growth than $A$. siliceus (Terquem).

\section{Ammodiscus siliceus asper (Terquem, 1863)}

$$
\text { (Pl. 4, Figs. 114, 117) }
$$

+1863 Involutina aspera Terquem, p. 221, pl. 10, fig. 21. 1936 Ammodiscus infimus (Strickland). Franke, p. 15, pl. 1, fig. 14. 1937 Ammodiscus incertus d'Orbigny. Bartenstein and Brand, p. 130, pl. 3 , fig. 5 ; pl. 4 , fig. 4 ; pl. 5 , fig. 5 .
1960 Involutina aspera Terquem. Bizon, p. 4, pl. 1, fig. 1.

1969 Ammodiscus asper (Terquem). Brouwer, p. 24, pl. 1, fig. 6.

Remarks. This subspecies is somewhat more coarsely agglutinated than $A$. siliceus siliceus (Terquem, 1863), but there are no other significant differences. The subspecies asper is common in the Sinemurian of France, England, and Germany, and also common in the late Pliensbachian and late Toarcian of eastern England and the North Sea (Copestake, unpubl. data)

\section{Glomospira pattoni Tappan, 1955}

(Pl. 4, Figs. 115-116)

+ 1955 Glomospira pattoni Tappan, p. 40, pl. 8, figs. 15-17.

Remarks. A long-ranging species, occurring from Lower to Upper Jurassic.

\section{Glomospira variabilis (Kübler and Zwingli, 1870)}

(Pl. 8, Figs. 184-186)

1865 Spirillina. Schwager, pl. 7, fig. 21.

+1870 Cornuspira variabilis Kübler and Zwingli, p. 33, pl. 4, fig. 4. 1886 Ammodiscus gordialis Parker and Jones. Haeusler, p. 24, pl. 3, figs. 11-12, 18-20, 22.

1890 Ammodiscus charoides Jones and Parker. Haeusler, p. 60, pl. 9, figs. 22-25.

1890 Ammodiscus gordialis Jones and Parker. Haeusler, p. 59, pl. 9, figs. 26-38; pl. 10, fig. 1 .

1941 Glomospira gordialis Jones and Parker. Frentzen, 307, pl. 1, figs. 15-17.

1960 Glomospira variabilis (Kübler and Zwingli). Seibold and Seibold, p. 324, text-fig. 2q-s.

1968 Glomospira variabilis (Kübler and Zwingli). Oesterle, p. 711, figs. 8-9, 10a-d.

1972 Glomospira pattoni Tappan. Dain, pl. 1, fig. 9.

1972 Glomospira variabilis (Kübler and Zwingli). Luterbacher, pl. 2, figs. 4-6.

1974 Glomospira charoides (Jones and Parker). Bartenstein, p. 686, pl. 2, figs. $45-50$.

1978 Glomospira variabilis (Kübler and Zwingli). Kuznetsova and Seibold, pl. 1, figs. 11-13; pl. 3, fig. 12 .

1980 Glomospira variabilis (Kübler and Zwingli). Sliter, pl. 1, figs. 9-10. Remarks. The species occurs from the Oxfordian to Tithonian (Lower Cretaceous?). It is very frequent in the Oxfordian of Switzerland and southwest Germany (sponge-algae bioherms). Typical color is yellow. Surface very finely agglutinated.

\section{Glomospira virgula (Kosyreva, 1972)}

$$
\text { (Pl. 8, Figs. 187-188) }
$$

+1972 Tolypammina virgula Kosyreva in Dain, p. 31, pl. 1, figs. 2-5. 1972 Glomospira sp. Luterbacher, pl. 2, fig. 7.

1974 Glomospira gordialis (Jones and Parker). Bartenstein, p. 686, pl. 2, figs. 51-54; pl. 3, figs. 1-3.

Remarks. More irregularly and more coarse agglutinated than $G$. variabilis (Kübler and Zwingli).

\section{Ammovertella? plicata (Terquem, 1886)}

(Pl. 8, Fig. 183)

+1886 Ammodiscus plicatus Terquem, p. 9, pl. 1, figs. 16-17

1941 Ammovertella plicata (Terquem). Frentzen, p. 303, pl. 1, figs. 8-9.

Distribution. Upper Middle Jurassic (France, southwest Germany).

\section{Tolypammina jurensis Franke, 1936}

(Pl. 2, Fig. 55)

+1936 Tolypammina jurensis Franke, p. 15, pl. 1, fig. 11 .

\section{?Tolypammina sp.}

(Pl. 1, Fig. 9)

1936 Hyperammina sp. Franke, p. 14, pl. 1, fig. 10.

2Tolypammina sp.

(Pl. 4, Fig. 118)

1936 Astrorhiza cretacea Franke, Franke, p. 10, pl. 1, fig. 1. 
?Tolypammina sp.

(Pl. 4, Fig. 120)

Remarks. It is unclear whether this specimen is a Tolypammina or coiled and belonging to another genus.

\section{Reophax helveticus (Haeusler, 1881)}

(Pl. 8, Fig. 196)

+1881 Dentalina helvetica Haeusler, p. 34, pl. 2, fig. 45.

1890 Reophax helvetica Haeusler, p. 28, pl. 3, figs. 15-17.

1944 Reophax scorpiurus Montfort. Frentzen, p. 329, pl. 18, figs. 6-8.

1968 Reophax helveticus (Haeusler). Oesterle, p. 715, text-figs. 13,

$14 \mathrm{~b}-\mathrm{c}, 15 \mathrm{k}-\mathrm{p}$.

1972 Reophax multilocularis Haeusler, Luterbacher, pl. 1, fig. 4.

1980 Reophax helveticus (Haeusler). Sliter, pl. 1, figs. 15-16.

Remarks. Abundant in the North Sea late Oxfordian (Copestake, unpubl. data).

\section{Reophax imperspicuus Seibold and Seibold, 1960}

(Pl. 8, Fig. 193)

+1960 Reophax imperspicuus Seibold and Seibold, p. 319, pl. 7, fig. 6, text-figs. 2c-e.

1960 Reophax variabilis Haeusler. Seibold and Seibold, p. 40, text-fig.

5.1 .

Remarks. A very irregularly shaped Reophax characteristic of the Oxfordian and lower Kimmeridgian of southwest Germany. Chambers not visible, wall coarsely to finely agglutinated, in general rather robust.

\section{Haplophragmoides barrowensis Tappan, 1951}

$$
\text { (Pl. 1, Fig. 11) }
$$

+1951 Haplophragmoides barrowensis Tappan, p. 1, pl. 1, fig. 5 .

Remarks. Has more chambers and a larger umbilicus than Haplophragmoides kingakensis Tappan.

\section{Haplophragmoides kingakensis Tappan, 1955} (Pl. 1, Fig. 10)

+ 1955 Haplophragmoides kingakensis Tappan, p. 43, pl. 10, figs. 1-6. Remarks. Found throughout the Jurassic.

\section{Haplophragmoides globigerinoides (Haeusler, 1882)}

$$
\text { (Pl. 8, Fig. 190) }
$$

+1882 Trochammina globigerinoides Haeusler, p. 352, pl. 15, figs. 8-9. 1890 Trochammina globigerinoides Haeusler, p. 66, pl. 10, figs. 20-23. 1960 Haplophragmoides globigerinoides (Haeusler). Seibold and Seibold, p. 326, text-fig. 5 f.

1968 Haplophragmoides globigerinoides (Haeusler). Oesterle, p. 727, text-figs. 23-24, $28 \mathrm{~b}$.

Remarks. This species is more coarsely agglutinated and has higher chambers than Haplophragmoides hyalinus (Haeusler).

\section{Haplophragmoides hyalinus (Haeusler, 1886)}

(Pl. 8, Fig. 191)

+1886 Trochammina hyalina Haeusler, pp. 3, 5, 27.

1890 Trochammina trulissata Brady?. Haeusler, p. 64, pl. 10, fig. 9.

1968 Haplophragmoides hyalinus (Haeusler). Oesterle, p. 729, text-

figs. $25 \mathrm{~b}, 26,28 \mathrm{c}$.

Remarks. Differs from all other Haplophragmoides species of the Jurassic in having a smooth, finely agglutinated surface and low chambers.

\section{Haplophragmium pictonicum Berthelin, 1879}

(Pl. 1, Fig. 12)

+1879 Haplophragmium pictonicum Berthelin, p. 26, pl. 1, figs 1-2. Remarks. A coarsely agglutinated species, distributed from the Hettangian (southwest-Germany) to the upper Pliensbachian (France, southwest Germany).

\section{Dorothia dumortieri (Schwager, 1866)}

(Pl. 8, Fig. 195)

+1866 Textularia dumortieri Schwager in Oppel, p. 309, text-fig. 14 . 1944 Textularia aff. conica d'Orbigny. Ströbel, pl. 12, figs. 16-17.

\section{Bigenerina arcuata Haeusler, 1890}

(Pl. 2, Figs. 57-58)

+1890 Bigenerina arcuata Haeusler, p. 73 , pl. 11, fig. 39; pl. 12, figs. 5-7.

1941 Bigenerina deceptoria Haeusler. Frentzen, p. 361, pl. 6, figs. 17-21.

1944 Bigenerina arcuata Haeusler. Frentzen, p. 335, pl. 18, fig. 24, 26, 28 .

1960 Gaudryinella deceptoria (Haeusler). Seibold and Seibold, p. 335, text-fig. 4d, o; pl. 8, fig. 12.

1961 Bigenerina $\mathrm{cf}$. nodosaria d'Orbigny. Gordon, p. 524, text-fig. $1(5)$.

1968 Bigenerina arcuata Haeusler. Oesterle, p. 742, text-figs. 37-39.

1972 Bigenerina arcuata Haeusler. Luterbacher, pl. 1, fig. 11.

Remarks. The specimens of Pl. 2, Figs. 57-58, are badly preserved and compressed in the biserial part or damaged.

\section{Bigenerina jurassica (Haeusler, 1890)}

(Pl. 2, Fig. 59)

+1890 Pleurostomella jurassica Haeusler, p. 77, pl. 12, figs. 14-22.

1944 Bigenerina arcuata Haeusler. Frentzen, p. 335, pl. 18, figs. 25, 27.

1968 Bigenerina jurassica (Haeusler). Oesterle, p. 745, text-figs. 40-42.

1972 Bigenerina jurassica Haeusler. Luterbacher, pl. 1, fig. 12.

Remarks. Differs from Bigenerina arcuata Haeusler in having a longer biserial stage and a finer agglutinated surface.

\section{Trochammina canningensis Tappan, 1955}

(PI. 8, Figs. 189, 192)

1886 Trochammina inflata Montfort. Haeusler, p. 28, pl. 3, fig. 28. +1955 Trochammina canningensis Tappan, p. 48, pl. 14, figs. 15-19. 1959 Trochammina globigeriniformis (Parker and Jones). Lloyd, p.

317 , pl. 5, fig. 31, text-fig. 5c.

1972 Haplophragmoides(?) sp. Luterbacher, pl. 1, figs. 13-15.

Remarks. The illustrated specimen has more chambers in the final whorl and appears higher spired than is usual for the species.

\section{Trochammina pulchra Ziegler, 1959}

(Pl. 2, Fig. 56)

1924 Haplophragmium canariense d’Orbigny. Klähn, p. 460, pl. 22, fig. 9.

+1959 Trochammina pulchra Ziegler, p. 94, pl. 2, figs. 6-8.

Remarks. This species has a flattened ventral side. The dorsal side is rounded, the chambers are very high, the umbilicus very narrow. Known from the Callovian of south Germany.

\section{Verneuilinoides tryphera Loeblich and Tappan, 1950}

(Pl. 2, Figs. 60-61)

+1950 Verneuilinoides tryphera Loeblich and Tappan, p. 42, pl. 11, fig. 16.

1974 Verneuilinoides neocomiensis (Mjatliuk). Kuznetsova, pl. 1, fig. 6. 1978 Verneuilinoides tryphera Loeblich and Tappan. Bhalla and Abbas, p. 172 , pl. 2, fig. 3 .

Remarks. Both specimens figured here are compressed: Fig. 60 in its longitudinal axis, Fig. 61 in the early chambers. They are triserial in their entire length, and the chambers are rounded in outline

\section{Ophthalmidium baccularis Issler, 1908}

(Pl. 4, Fig. 119)

+ 1908 Ophthalmidium baccularis Issler, p. 44, pl. 1, fig. 25 .

Remarks. An Ophthalmidium of the upper Sinemurian of Southwest Germany. Chambers not visible externally in outline.

\section{Ophthalmidium carinatum (Kübler and Zwingli, 1866)}

$$
\text { (Pl. 1, Fig. 14) }
$$

+1866 Oculina carinata Kübler and Zwingli, p. 14, pl. 2, fig. 19 . 1875 Spiroloculina concentrica Terquem and Berthelin, p. 80, pl. 7 , figs. 1-4.

1936 Spirophthalmidium concentricum (Terquem and Berthelin). Franke, p. 123, pl. 12, fig. 17. 
1937 Spirophthalmidium concentricum (Terquem and Berthelin). Bartenstein and Brand, p. 181, pl. 2B, figs. 37-38; pl. 4, fig. 16; pl. 5, fig. 71 ; pl. 8, fig. 36 ; pl. 13, fig. 21 ; pl. 15A, fig. 39 .

1941 Ophthalmidium carinatum (Kübler and Zwingli). Macfadyen, p. 23, pl. 1, fig. 12.

1968 Ophthalmidium carinatum (Kübler and Zwingli). Welzel, p. 9, pl. 3 , fig. 1 .

1969 Ophthalmidium carinatum (Kübler and Zwingli). Brouwer, p. 26, pl. 2, fig. 8 .

Distribution. Lower Sinemurian to Oxfordian.

Ophthalmidium liasicum (Kübler and Zwingli, 1866) (Pl. 1, Fig. 13)

+1866 Oculina liasica Kübler and Zwingli, p. 11, pl, 1, fig. 24 . 1870 Ophthalmidium liasicum Kübler and Zwingli, Kübler and Zwingli, p. 11 , pl. 1, fig. 11 .

1886 Ophthalmidium orbiculare Burbach (1886b), p. 499, pl. 5, figs. 3-6.

1886 Ophthalmidium ovale Burbach (1886b), p. 499, pl. 5, figs. 7-12.

1936 Ophthalmidium orbiculare Burbach. Franke, p. 122, pl. 12, fig. 12.

1936 Ophthalmidium ovale Burbach. Franke, p. 123, pl. 12, fig. 13.

1937 Ophthalmidium ovale Burbach. Bartenstein and Brand, p. 181, pl. 4 , fig. 18.

1937 Ophthalmidium orbiculare Burbach. Bartenstein and Brand, p. 181 , pl. 2 A, fig. 22 ; pl. 4 , fig. 17.

1968 Ophthalmidium orbiculare Burbach. Welzel; p. 8, pl. 3, fig. 2. 1968 Ophthalmidium ovale Burbach. Welzel, p. 8, pl. 3, fig. 3.

1968 Ophthalmidium nubeculariformis Haeusler. Welzel, p. 9, pl. 3, fig. 4 .

1969 Ophthalmidium orbiculare Burbach. Brouwer, p. 27, pl. 2, figs. 9-10.

Distribution. Hettangian to lowermost Toarcian.

Ophthalmidium strumosum (Gümbel, 1862)

(Pl. 8, Figs. 194, 197)

+1862 Guttulina strumosa Gümbel, 227, pl. 4, figs. 13-14.

1865 Guttulina strumosa Gümbel. Schwager, p. 137, pl. 7, fig. 9.

1917 Ophthalmidium birmenstorfenis Kübler and Zwingli. Paalzow, p. 220 , pl. 41 , figs. 17-19.

1932 Spirophthalmidium carinatum Kübler and Zwingli. Paalzow, p. 99 , pl. 5, figs. 7-10.

1955 Ophthalmidium strumosum (Gümbel). Seibold and Seibold, p. 102 , text-fig. $3 \mathrm{~h}$, i.

1962 Spirophthalmidium strumosum (Gümbel). Bastien and Sigal, p. 92 , pl. 5 , figs. $22-23$.

Distribution. Oxfordian (France, Switzerland, South Germany).

\section{Ophthalmidium sp.}

(Pl. 2, Fig. 62)

Remarks. An Ophthalmidium which resembles Ophthalmidium rotula Lalicker, 1956, but shows no chamber externally.

\section{Triloculina meotica Loeblich and Tappan, 1946}

(Pl. 2, Figs. 63-64)

+1946 Triloculina meotica Loeblich and Tappan, p. 247, pl. 3, figs. 1-3.

1978 Quinqueloculina sp. C. Bhalla and Abbas, p. 173, pl. 4, fig. 3. 1980 Triloculina meotica Loeblich and Tappan. Sliter, pl. 5, fig. 16.

Remarks. This lower Cretaceous species shows no sculpture and considerable variability.

\section{Nodosaria alemannica Frentzen, 1941}

(Pl. 5, Fig. 125)

+ 1941 Nodosaria alemmanica Frentzen, p. 315, pl. 2, fig. 7 .

1967 Nodosaria cf. columnaris Franke. Ruget and Sigal, p. 62, pl. 8, fig. 5 .

\section{Nodosaria apheilolocula Tappan, 1955}

(Pl. 5, Fig. 130)

+1875 Nodosaria incerta Terquem and Berthelin, p. 18, pl. 1, fig. 15. 1937 Nodosaria hirsuta d'Orbigny. Bartenstein and Brand, p. 145, pl. 4 , fig. 39.
1941 Nodosaria hispida Reuss. Macfadyen, p. 63, pl. 4, fig. 64. 1955 Nodosaria apheilolocula Tappan, p. 68, pl. 24, figs. 6-7.

Remarks. This species is very small and has little spines on the chamber surfaces which cannot be seen in Fig. 130 because they are recrystallized. Sinemurian to lowermost Toarcian.

\section{Nodosaria caudata Frentzen, 1941

$$
\text { (Pl. 1, Fig. 15) }
$$

1936 Nodosaria hybrida (Terquem and Berthelin). Franke, p. 42, pl. 4, fig. 2.

+1941 Nodosaria caudata Frentzen, p. 317, pl. 2, fig. 32.

1968 Nodosaria regularis candela Franke. Welzel, p. 11, pl. 1, fig. 18.

\section{Nodosaria claviformis Terquem, 1866}

(Pl. 5, Fig. 127)

+1866 Nodosaria claviformis Terquem, p. 477, pl. 19, figs. 17-18.

1960 Nodosaria claviformis Terquem. Bizon, p. 9, pl. 3, fig. 5; pl. 4, fig. 12.

\section{Nodosaria clavula (Terquem, 1870)}

(Pl. 8, Fig. 201)

+1870 Dentalina clavula Terquem (1870b), p. 264, pl. 28, fig. 4 .

Nodosaria geniculata (Terquem and Berthelin, 1875) (PI. 4, Fig. 121)

+1875 Dentalina geniculata Terquem and Berthelin, p. 32, pl. 2, fig. 22. 1957 Nodosaria dispar Franke. Nørvang, p. 356, fig. 80.

\section{Nodosaria incongrua Kübler and Zwingli, 1866}

(Pl. 8, Fig. 199)

?1865 Nodosaria manubrium Schwager, p. 99, pl. 2, fig. 14 +1866 Nodosaria incongrua Kübler and Zwingli, p. 13, pl. 2, fig. 17. 1870 Nodosaria incongrua Kübler and Zwingli, p. 15, pl. 2, fig. 1 (Bajoc.).

\section{Nodosaria kunzi Paalzow, 1917}

(Pl. 8, Fig. 200)

+ 1917 Nodosaria kunzi Paalzow, p. 229, pl. 42, fig. 24. 1922 Nodosaria fontinensis Terquem. Paalzow, p. 16, pl. 2, fig. 3. 1924 Nodosaria prima d’Orbigny. Klähn, p. 457, pl. 23, fig. 15. 1970 Nodosaria jurassica Gümbel. Winter, p. 32, pl. 4, fig. 112. 1978 Nodosaria plicatilis Wiśniowski. Munk, p. 39, pl. 1, fig. 6.

Remarks. The holotype of this species has more ribs than the specimen of Fig. 200. It resembles Nodosaria corallina Gümbel, 1862.

\section{Nodosaria lagenoides Wiśniowski, 1890}

(Pl. 8, Fig. 198)

1890 Lagena sulcata Walker and Jacob. Haeusler, p. 87, pl. 13, figs. 27-29.

+1890 Nodosaria lagenoides Wiśniowski, p. 194, pl. 1, fig. 25.

1960 Nodosaria nodosaroides Paalzow. Seibold and Seibold, p. 369, text-fig. $7 \mathrm{~b}$

1978 Nodosaria lagenoides Wiśniowski. Munk, p. 39, pl. 1, fig. 7. 1980 Lagena sulcata (Walter and Jacob). Sliter, pl. 8, figs. 8-10.

\section{Nodosaria lamellosa (Terquem, 1866)}

(Pl. 5, Fig. 126)

+1866 Dentalina lamellosa Terquem, p. 410, pl. 15, fig. 16 .

1936 Nodosaria mitis Terquem and Berthelin. Franke, p. 45, pl. 4, fig. 11a.

1957 Nodosaria columnaris Franke. Nørvang, p. 351, figs. 75-76.

\section{Nodosaria multicostata Terquem and Berthelin, 1875}

$$
\text { (Pl. 5, Fig. 131) }
$$

1875 Nodosaria variabilis Terquem and Berthelin, p. 20, pl. 1 , fig. 19 (preoccupied).

+1875 Nodosaria multicostata Terquem and Berthelin, p. 20, pl, 1, fig. 20.

1937 Nodosaria mutabilis Terquem. Bartenstein and Brand, p. 148, pl. 4 , fig. 38 ; pl. 5, fig. 23 . 
Nodosaria ornata (Terquem, 1858)

(Pl. 4, Fig. 122)

+1858 Dentalina ornata Terquem, p. 44, pl. 2 , fig. 13.

\section{Nodosaria perlata Frentzen, 1941}

(Pl. 4, Fig. 123)

+1941 Nodosaria perlata Frentzen, p. 321, pl. 2, fig. 23.

Remarks. A rare species from the Pliensbachian (Southwest Germany).

\section{Nodosaria regularis Terquem, 1862}

(Pl. 5, Fig. 129)

+1862 Nodosaria regularis Terquem, p. 436, pl. 5, fig. 12 .

1936 Nodosaria regularis Terquem. Franke, p. 41, pl. 3, fig. 19.

1936 Nodosaria crispata Terquem. Franke, p. 41, pl. 3, fig. 20.

1968 Nodosaria apheilolocula Tappan. Welzel, p. 11, pl. 1, fig. 25 .

\section{Nodosaria sexcostata Terquem, 1858}

(Pl. 4, Fig. 124)

+1858 Nodosaria sexcostata Terquem, p. 28 , pl. 1, fig. 5 .

1866 Dentalina paucicosta Terquem, p. 483, pl. 19, fig. 22.

1866 Dentalina radiata Terquem, p. 490 , pl. 20, fig. 5.

1875 Dentalina mitis Terquem and Berthelin, p. 28, pl. 2, fig. 9.

1875 Dentalina demissa Terquem and Berthelin, p. 28, pl. 2, fig. 10.

1875 Dentalina oculina Terquem and Berthelin, p. 31, pl. 2, fig. 20.

1936 Nodosaria oculina (Terquem and Berthelin). Franke, p. 49, pl. 4, fig. 21.

1936 Nodosaria oculina (Terquem and Berthelin) f. vermicularis Franke, p. 449, pl. 4, fig. 22.

1937 Nodosaria mitis (Terquem and Berthelin). Bartenstein and Brand, p. 145, pl. 3, fig. 18; pl. 5, fig. 24.

1937 Nodosaria oculina (Terquem and Berthelin). Bartenstein and Brand, p. 147 , pl. 5, fig. 25.

1968 Nodosaria sexcostata sexcostata Terquem. Welzel, p. 14, pl. 1, fig. 30 .

1968 Nodosaria sexcostata radiata (Terquem). Welzel, p. 14, pl. 1, fig. 31 .

1968 Nodosaria sexcostata mitis (Terquem and Berthelin). Welzel, p. 15, pl. 1, fig. 32 .

1968 Nodosaria sexcostata oculina (Terquem and Berthelin). Welzel, p. 15 , pl. 1, figs. 33-34.

Remarks. A frequent species in the Lias of Europe, which ranges from Hettangian to lowermost Toarcian.

\section{Nodosaria simplex (Terquem, 1858)} (Pl. 5, Fig 128)

+1858 Dentalina simplex Terquem, p. 39 , pl. 2 , fig. 5 .

1936 Nodosaria simplex (Terquem). Franke, p. 44, pl. 4, fig. 6.

1968 Nodosaria simplex simplex (Terquem). Welzel, p. 12, pl. 1, fig. 21 .

1968 Nodosaria simplex tenuissima Franke. Welzel, p. 13, pl. 1, fig. 22.

Remarks. A slender, simple, smooth Nodosaria, that ranges from Lower to Upper Jurassic.

\section{Dentalina alternans Terquem, 1870}

(Pl. 9, Fig. 204)

+1870 Dentalina alternans Terquem (1870b), p. 261, pl. 27, figs. 23-25.

\section{Dentalina biloculina (Franke, 1936)}

$$
\text { (P1. 5, Fig. 134) }
$$

+1936 Nodosaria biloculina Franke, p. 42, pl. 3, fig. 23.

Remarks. Because of its asymmetrical chambers, this species belongs to the genus Dentalina.

\section{Dentalina crenata Schwager, 1865}

(P1. 2, Fig. 65)

+ 1865 Dentalina crenata Schwager, P. 109, pl. 3, figs. 19, 25 . 1932 Dentalina oligostegia Reuss. Paalzow, p. 113, pl. 7, fig. 23.

1960 Dentalina crenata Schwager. Seibold and Seibold, p. 59, pl. 5, fig. 14 .
Dentalina cushmani Paalzow, 1932

(Pl. 9, Fig. 202)

+1932 Dentalina cushmani Paalzow, p. 112, pl. 7, figs. 18, 22.

Dentalina digitalis Franke, 1936

(Pl. 5, Fig. 142)

+1936 Dentalina digitalis Franke, p. 32, pl. 2, fig. 26.

Dentalina glandulinoides Franke, 1936

(Pl. 5, Fig. 141)

+1936 Dentalina glandulinoides Franke, p. 28, pl. 2, fig. 14 .

\section{Dentalina gyrosa Terquem, 1866}

(Pl. 1, Fig. 17)

+ 1866 Dentalina gyrosa Terquem, p. 407, pl. 15, fig. 10 .

Remarks. This species probably belongs to the group of dentalina matutina. d'Orbigny, 1850.

\section{Dentalina haeusleri Paalzow, 1917}

(PI. 9, Fig. 203)

+ 1917 Dentalina häusleri Paalzow, p. 232, pl. 43, figs. 12-13.

Dentalina integra (Kübler and Zwingli, 1866)

(Pl. 5, Figs. 136-137)

+1866 Vaginulina integra Kübler and Zwingli, p. 8, pl. 1, fig. 2.

1870 Vaginulina integra Kübler and Zwingli, p. 5, pl. 1, fig. 5.

1968 Dentalina integra (Kübler and Zwingli). Welzel, p. 27, pl. 1,

fig. 57 .

\section{Dentalina jurensis (Gümbel, 1862)}

$$
\text { (Pl. 2, Fig. 153) }
$$

+ 1862 Vaginulina jurensis Gümbel, p. 220, pl. 3, fig. 14.

1865 Dentalina mutabilis Schwager, p. 103, pl. 2, fig. 24.

1870 Dentalina intorta Terquem (1870b), p. 262, pl. 27, figs. 26-34.

1932 Dentalina peracuta Reuss. Paalzow, p. 113, pl. 7, fig. 19.

1955 Dentalina jurensis (Gümbel). Seibold and Seibold, p. 112, pl. 13, fig. 9.

1960 Dentalina peracuta Reuss. Seibold and Seibold, p. 60, pl. 4, fig. 14 .

\section{Dentalina nodigera Terquem and Berthelin, 1875}

(Pl. 5, Fig. 135)

+1875 Dentalina nodigera Terquem and Berthelin, p. 25, pl. 1, fig. 31 .

1936 Dentalina nodigera Terquem and Berthelin. Franke, p. 26, pl. 2, fig. 7 .

1968 Dentalina varians Terquem. Welzel, p. 24 , pl. 3, figs. 17-18.

Remarks. An inflated Dentalina, which ranges from lower Pliensbachian to lowermost Toarcian (Germany, France).

\section{Dentalina plebeia Terquem, 1870}

(Pl. 2, Fig. 67)

+1870 Dentalina plebeia Terquem (1870b), p. 267, pl. 29, figs. 3-11.

Dentalina aff. pseudoarcuata E. and I. Seibold, 1960

$$
\text { (Pl. 2, Fig. 68) }
$$

aff. 1960 Dentalina pseudoarcuata E. and I. Seibold, p. 360, textfig. 60 .

1967 Dentalina aff. incerta Terquem. Groiss, p. 14, pl. 1, fig. 35.

Remarks. Differs from Dentalina pseudoarcuata E. and I. Seibold in not having oblique sutures.

\section{Dentalina sculpta Terquem, 1866}

(Pl. 5, Fig. 133)

+1866 Dentalina sculpta Terquem, p. 484, pl. 19, fig. 24 . 1936 Dentalina tenuistriata Terquem. Franke, p. 35, pl. 3, fig. 7c.

1968 Dentalina tenuistriata tenuistriata Terquem. Welzel, p. 29, pl. 3, fig. 21 . 
Dentalina suboligostegia Franke, 1936

(Pl. 5, Fig. 139)

+1936 Dentalina suboligostegia Franke, p. 25, pl. 2, fig. 1 .

1936 Dentalina tortilis Franke, p. 29, pl. 2, fig. 19.

1968 Dentalina submucronata Franke. Welzel, p. 22, pl. 1, figs. 52-53.

Dentalina subsiliqua Franke, 1936

(Pl. 5, Fig. 138)

+1936 Dentalina subsiliqua Franke, p. 30, pl. 2, fig. 21.

Dentalina subulata Franke, 1936

(Pl. 5, Figs. 147-148)

+1936 Dentalina subulata Franke, p. 27, pl. 2, fig. 11 .

1968 Dentalina subulata Franke. Welzel, p. 21, pl. 1, fig. 51.

\section{Dentalina cf. terquemi d'Orbigny, 1849}

(Pl. 5, Fig. 140)

cf. 1850 Dentalina terquemi d'Orbigny, p. 242.

cf. 1936 Dentalina terquemi d'Orbigny. Macfadyen, p. 149, pl. 1, fig. 257.

Remarks. The specimen described here has a nearly circular section and no chamber incisions like Dentalina terquemi d'Orbigny.

\section{Dentalina teutoburgensis Franke, 1936}

(Pl. 1, Fig. 16; Pl. 5, Fig. 132)

+1936 Dentalina teutoburgensis Franke, p. 33, pl. 3, fig. 3 .

1968 Dentalina matutina funiculosa Terquem. Welzel, p. 28, pl. 2, fig. 6 (7?).

\section{Dentalina torta Terquem, 1858}

$$
\text { (Pl. 5, Fig. 143) }
$$

+1858 Dentalina torta Terquem, p. 39 , pl. 2 , fig. 6 .

1936 Dentalina pseudocommunis Franke, p. 30, pl. 2, fig. 20.

1968 Dentalina torta Terquem. Welzel, p. 22, pl. 1, fig. 49.

\section{Dentalina turgida Schwager, 1865}

(PI. 2, Fig. 69)

+1865 Dentalina turgida Schwager, p. 100, pl. 2, fig. 19; pl. 3, figs. 6, $11,20$.

1890 Nodosaria (Dentalina) mucronata Neugebohren. Haeusler, p. 100 , pl. 13, fig. 102.

1932 Marginulina apiculata Reuss. Paalzow, p. 106, pl. 7, figs. 1-2.

1956 Dentalina turgida Schwager. Seibold and Seibold, p. 135, textfig. 4r.

\section{Dentalina vetustissima d'Orbigny, 1850}

(Pl. 5, Figs. 144-146)

+1850 Dentalina vetustissima d'Orbigny, p. 242 .

1936 Dentalina vetustissima d'Orbigny. Macfadyen, p. 150, pl. 1 , fig. 261.

1936 Dentalina exilis Franke, p. 31, pl. 2, fig. 25.

Remarks. An elongated Dentalina with straight, but weak chamber incisions.

Dentalina sp.

(Pl. 2, Fig. 70)

Remarks. A smooth Dentalina without any constrictions or visible sutures.

Dentalina sp. A

(Pl. 2, Fig. 71)

Remarks. A very slender, smooth Dentalina, without constrictions.

\section{Astacolus carinocostata (Deecke, 1884)}

(Pl. 2, Fig. 73)

+ 1884 Cristellaria carinocostata Deecke, p. 43, pl. 2, figs. 5-5a.

1978 Lenticulina (Astacolus) interrumpa (Blank). Munk, p. 45, pl. 3,

fig. 8 .

Remarks. This species shows broad constrictions of the chambers and a keel.
Astacolus deformis (Bornemann, 1854)

(Pl. 6, Fig. 155)

+1854 Cristellaria deformis Bornemann, p. 41, pl. 4, fig. 35 .

Astacolus semiincisus (Terquem and Berthelin, 1875)

(Pl. 1, Fig. 18; Pl. 6, Fig. 156)

+1875 Cristellaria semi-incisa Terquem and Berthelin, p. 48, pl. 4, fig. 9.

1969 Astacolus primus (d'Orbigny). Brouwer, p. 30, pl. 6, fig. 6.

Astacolus spongiphilus (Gümbel, 1862)

(Pl. 2, Fig. 72)

+1862 Cristellaria spongiphila Gümbel, p. 225, pl. 3, fig. 26.

1955 Lenticulina (Astacolus) prima franconica (Gümbel). Seibold and Seibold, p. 109, text-fig. 3k, 1; pl. 13, fig. 6.

1956 Lenticulina (Astacolus) fraasi (Schwager). Seibold and Seibold, p. 116 , text-fig. $5 \mathrm{~m}$, $\mathrm{n}$.

1967 Astacolus primus franconicus (Gümbel). Groiss, p. 28, pl. 3, fig. 91.

1970 Astacolus primus franconicus (Gümbel). Winter, p. 16, pl. 2, fig. 60 .

Astacolus stillus (Terquem, 1866)

(Pl. 6, Fig. 157)

+1866 Cristellaria stilla Terquem, p. 517, pl. 22, fig. 7 .

1941 Cristellaria similis Terquem. Macfadyen, p. 34, pl. 2, fig. 26.

Astacolus turgidus (Schwager, 1865)

(Pl. 2, Fig. 74)

+1865 Cristellaria turgida Schwager, p. 127, pl. 6, fig. 4 .

1865 Cristellaria informis Schwager, p. 128, pl. 6, fig. 8.

1890 Cristellaria cultrata Monfort. Haeusler, p. 114, pl. 15, figs. 4-5.

1956 Lenticulina (Astacolus) matutina informis (Schwager). Seibold and Seibold, p. 119, pl. 7, fig. 13, text-fig. 4e-g.

1972 Lenticulina involvens Wiśniowski). Luterbacher, pl. 3, figs. 7-8.

\section{Astacolus cf. turgidus (Schwager, 1865)}

$$
\text { (PI. 2, Fig. 75) }
$$

Remarks. Differs from Astacolus turgidus (Schwager) in having lower chambers and stronger constrictions at the sutures.

\section{Astacolus varians (Bornemann, 1854)}

(Pl. 6, Fig. 149)

+1854 Cristellaria varians Bornemann, p. 41, pl. 4, figs. 32-34.

1936 Cristellaria (Lenticulina) varians Bornemann, var. suturalis-costata Franke, p. 113, pl. 11, fig. 13.

1937 Cristellaria varians Bornemann. Bartenstein and Brand, p. 176, pl. 2B, fig. 32

1963 Lenticulina (Astacolus) varians (Bornemann). Rabitz, p. 203, pl. 16 , figs. $2,3,6$.

1975 Lenticulina varians (Bornemann). Jendryka-Fuglewitz, p. 136, pl. 2, figs. 1-2.

Remarks. A very common species in the Lias and rather variable, often with a keel and weak constrictions at the sutures.

\section{Citharina colliezi (Terquem, 1866)}

(PI. 2, Fig. 76)

+1866 Marginulina colliezi Terquem, p. 430, pl. 17, fig. 10 .

1868 Marginulina flabelloides Terquem, p. 102, pl. 6, figs. 1-30.

1917 Vaginulina flabelloides Terquem. Paalzow, p. 238, pl. 45, figs. 8-9.

1924 Vaginulina flabelloides Terquem. Klähn, p. 29. pl. 1, fig. 16 1962 Citharina flabelloides (Terquem). Cordey, p. 385, pl. 47, fig. 17.

1971 Citharina colliezi (Terquem). Wernli, p. 316, pl. 2, figs. 13-17; pl. 3 , figs. 1-2.

1971 Citharina "colliezi-heteropleura". Wernli, p. 317, pl. 2, figs. 23-26.

1978 Vaginulina flabelloides (Terquem). Munk, p. 49, pl. 2, fig. 1. Distribution. The species is found from lower Toarcian to Oxfordian. 


\section{Citharina inconstans (Terquem, 1868)}

(pl. 2, Fig. 77)

+1868 Marginulina inconstans Terquem, p. 66, pl. 2, figs. 1-12. Remarks. The species belongs to the group of Citharina colliezi (Terquem) but differs in having coarser, more irregular ribs.

\section{Frondicularia major Bornemann, 1854}

$$
\text { (Pl. 1, Fig. 21) }
$$

+1854 Frondicularia major Bornemann, p. 36, pl. 3, fig. 21.

?1854 Frondicularia intumescens Bornemann, p. 36, pl. 3, fig. 19. 1886 Frondicularia carinata Burbach (1886a), p. 47, pl. 12, figs. $17-20,29$.

1886 Frondicularia elliptica Burbach (1886a), p. 48, pl. 1, figs. 21-26; pl. 2 , fig. 37.

1886 Frondicularia lata Burbach (1886a), p. 48, pl. 1, figs. 27-28, 30; pl. 2, figs. 31-32.

1936 Frondicularia major Bornemann. Franke, p. 68, pl. 7, fig. 2.

1937 Frondicularia major Bornemann. Bartenstein and Brand, p. 155, pl. 5 , fig. 68 .

1963 Frondicularia major Bornemann. Rabitz, p. 208, pl. 17, figs. 18-20.

1968 Frondicularia major Bornemann. Welzel, p. 37, pl. 2, fig. 20.

1968 Lingulina sacculus (Terquem). Welzel, p. 35, pl. 3, fig. 38.

Distribution. In Germany and France the species seems to be restricted to the lower Pliensbachian and the lowermost Toarcian.

\section{Frondicularia paradoxa Berthelin, 1879}

(Pl. 6, Figs. 150-151)

+1879 Frondicularia paradoxa Berthelin, p. 33, pl. 1, figs. 12-15, 15bis, 16-17.

1936 Flabellina paradoxa Berthelin. Franke, p. 91, pl. 9, figs. 10-11. 1936 Falsopalmula kuhni Franke, p. 92, pl. 9, fig. 14.

1937 Flabellina paradoxa (Berthelin). Bartenstein and Brand, p. 168, pl. 4 , fig. 63.

1957 Flabellina paradoxa Berthelin. Barnard, p. 173, text-fig. 2G-J. 1970 Berthelinella paradoxa (Berthelin). Fuchs, p. 110, pl. 8, fig. 7. ?1970 Sieberina virgata Fuchs, p. 107, pl. 8, figs. 11, 14; pl. 10, figs. A-D.

$? 1970$ Sieberina sagitta Fuchs, p. 107, pl. 8, fig. 18

Distribution. This species occurs in North and South Germany only in the lower Pliensbachian, in Austria in the ?Sinemurian (Fuchs, 1970), and in Britain in the Sinemurian-Toarcian (Copestake and Johnson, 1981).

\section{Frondicularia sacculus Terquem, 1866}

$$
\text { (Pl. 2, Fig. 22) }
$$

+1866 Frondicularia sacculus Terquem, p. 482, pl. 19, fig. 20.

1936 Frondicularia sacculus Terquem. Franke, p. 68, pl. 7, fig. 4 .

1937 Frondicularia sacculus Terquem. Bartenstein and Brand, p. 154, pl. 4 , fig. 46.

1967 Frondicularia major Bornemann. Ruget and Sigal, p. 48, pl. 4, fig. 14.

1967 Frondicularia nitida Terquem. Ruget and Sigal, p. 49, pl. 4, fig. 12.

1967 Frondicularia $\mathrm{cf}$. intumescens Bornemann. Ruget and Sigal, p. 49, pl. 4, fig. 13.

1967 Frondicularia cf. nitida Terquem. Ruget and Sigal, p. 49, pl. 4, fig. 16.

Remarks. A smooth Frondicularia, related to $F$ major Bornemann, which occurs from the Sinemurian to the upper Pliensbachian.

\section{Frondicularia squamosa Terquem and Berthelin, 1875}

$$
\text { (Pl. 6, Fig. 154) }
$$

+1875 Frondicularia squamosa Terquem and Berthelin, p. 37, pl. 3, fig. 3 .

1937 Frondicularia mesoliassica Brand in Bartenstein and Brand, p. 158 , pl. 4 , fig. 66 , text-fig. 16 .

1941 Frondicularia sulcata var. squamosa Terquem and Berthelin. Macfadyen, p. 61 , pl. 4 , fig. 61 .

1968 Frondicularia squamosa (Terquem and Berthelin). Welzel, p. 40, pl. 3, fig. 34.

1969 Frondicularia bicostata d'Orbigny. Brouwer, p. 35, pl. 5, fig. 7.
1970 Frondicularia muelensis Mouterde and Ruget, p. 92, pl. 3, figs. 19-32; pl. 4, figs. 1-6.

1981 Ichthyolaria squamosa. Hohenegger, pl. 2, table 5, text-fig. 8.

Remarks. Frondicularia squamosa Terquem and Berthelin has somewhat finer ribs and is more slender than Frondicularia terquemi d'Orbigny.

\section{Frondicularia terquemi terquemi d'Orbigny, 1850}

(Pl. 6, Fig. 152)

+1850 Frondicularia terquemi d'Orbigny, p. 241.

1850 Frondicularia bicostata d'Orbigny, p. 242.

1876 Frondicularia terquemi d'Orbigny. Blake in Tate and Blake, p. 468 , pl. 19, fig. 22 .

1936 Frondicularia terquemi d'Orbigny. Macfadyen, p. 149, pl. 1, fig. 255.

1936 Frondicularia bicostata d'Orbigny. Macfadyen, p. 149, pl. 1, fig. 256.

1936 Frondicularia bicostata d'Orbigny. Franke, p. 70, pl. 7, fig. 9.

1936 Frondicularia baueri Burbach. Franke, p. 70, pl. 7, fig. 10.

1936 Frondicularia terquemi d'Orbigny. Franke, p. 69, pl. 7, fig. 11.

1937 Frondicularia bicostata d'Orbigny. Bartenstein and Brand, p. 158 , pl. 3 , fig. 35 ; pl. 4 , fig. 48 .

1968 Frondicularia terquemi bicostata d'Orbigny. Bartenstein and Brand, p. 158, pl. 3, fig. 35; pl. 4, fig. 48.

1981 Ichthyolaria terquemi (d'Orbigny). Hohenegger, pl. 2, table 5, text-fig. 8.

Remarks. A species common in the upper Sinemurian and the Pliensbachian.

\section{Frondicularia terquemi dubia Bornemann, 1854}

(Pl. 6, Fig. 153)

+1854 Frondicularia dubia Bornemann, p. 37, pl. 3, fig. 23.

1936 Frondicularia dubia Bornemann. Franke, p. 72, pl. 7, fig. 6.

1937 Frondicularia dubia Bornemann. Bartenstein and Brand, p. 158, pl. 3, fig. 41 .

1957 Spandelina terquemi dubia (Bornemann). Nørvang, p. 344, figs. 60-61.

1957 Frondicularia sulcata plexus, form E. Barnard, p. 175, pl. 1, fig. E; pl. 2, Figs. 10, 22.

1968 Frondicularia terquemi dubia Bornemann. Welzel, p. 26, pl. 2, fig. 26.

1969 Frondicularia bicostata d'Orbigny. Brouwer, p. 35, pl. 58, figs. 3, 22.

1970 Frondicularia dubia Bornemann. Mouterde and Ruget, p. 90, pl. 2, figs. 27-28.

1970 Frondicularia gr. dubia Bornemann. Mouterde and Ruget, p. 90, pl. 2, figs. 29-30.

1981 Ichthyolaria terquemi. Hohenegger, pl. 2, table 5, text-fig. 8.

\section{Lenticulina gottingensis (Bornemann, 1854)}

$$
\text { (Pl. 6, Fig. 159) }
$$

+1854 Robulina gottingensis Bornemann, p. 43, pl. 4, figs. 40-41. 1936 Cristellaria (Lenticulina) gottingensis Bornemann. Franke, p. 116, pl. 11, fig. 22.

1957 Lenticulina gottingensis (Bornemann). Nørvang, p. 382, fig. 168.

1963 Lenticulina gottingensis (Bornemann). Rabitz, p. 202, pl. 16, fig. 4.

Remarks. A thick, smooth Lenticulina without keel, typical for lower Pliensbachian to lowermost Toarcian.

\section{Lenticulina hebetata (Schwager, 1865)}

(Pl. 9, Figs. 207, 209)

+1865 Cristellaria hebetata Schwager, p. 134, pl. 7, fig. 2 .

1953 Lenticulina (Lenticulina) muensteri (Roemer). Seibold and Seibold, p. 49 , pl. 4 , fig. 8.

1955 Lenticulina (Lenticulina) muensteri (Roemer). Seibold and Seibold, p. 104, text-fig. 4a-c.

1956 Lenticulina meunsteri hebetata (Schwager). Seibold and Seibold, p. 122 , text-fig. $4-\mathrm{k}, 1$.

1962 Lenticulina muensteri (Roemer). Cordey, p. 378, pl. 46, fig. 1. 1970 Lenticulina muensteri (Roemer). Winter, p. 25, pl. 3, fig. 93.

1972 Lenticulina muensteri (Roemer). Luterbacher, pl. 3, figs. 11-12. 
Remarks. Cross section thicker than that of Lenticulina gottingensis. Surface smooth, without any constrictions, no keel.

\section{Lenticulina inermis (Terquem, 1862)}

$$
\text { (Pl. 6, Fig. 158) }
$$

+1862 Cristellaria inermis Terquem, p. 447, pl. 6, fig. 5 .

\section{Lenticulina oolithica (Terquem, 1876)}

(Pl. 9, Figs. 206, 211)

+1876 Robulina oolithica Terquem, p. 496, pl. 17, fig. 10 .

1954 Lenticulina muensteri (Roemer). Bielecka and Pozaryski, p. 33, pl. 4 , fig. 12.

1971 Lenticulina muensteri (Roemer). Wernli, p. 321, pl. 4, fig. 21.

Remarks. Chamber sutures visible, with a keel, not so thick as Lenticulina hebetata (Schwager).

\section{Lenticulina polygonata (Franke, 1936)}

$$
\text { (Pl. 1, Fig. 23) }
$$

+1936 Cristellaria (Lenticulina) polygonata Franke, p. 118, pl. 12, figs. 1-2.

1968 Lenticulina muensteri polygonata (Franke). Welzel, p. 42, pl. 2, fig. 38.

1975 Lenticulina polygonata (Franke). Jendryka-Fuglewitz, p. 137, pl. 2, figs. 7-8.

Remarks. Faint to distinct polygonal outline, sutures visible, straight. A typical species of Pliensbachian to Toarcian age.

\section{Lenticulina? pusilla (Schwager, 1865)}

$$
\text { (P1. 9, Fig. 208) }
$$

+1865 Rotalia pusilla Schwager, p. 141, pl. 7, fig. 20.

1953 Gyroidina? sp. E. and I. Seibold, p. 70, pl. 6, fig. 10.

1956 Rotalia pusilla Schwager. Seibold and Seibold, p. 104.

Remarks. A symmetrical ?Lenticulina with distinct, broad constrictions in its outline, chamber sutures visible.

\section{Lenticulina quenstedti (Gümbel, 1862)}

$$
\text { (Pl. 9, Fig. 205) }
$$

+1862 Cristellaria quenstedti Gümbel, p. 226, pl. 4, fig. 2 .

1932 Lenticulina quenstedti Gümbel. Paalzow, p. 102, pl. 6, figs. 3-5. 1953 Lenticulina quenstedti (Gümbel). Seibold and Seibold, p. 49, pl. 4 , fig. 4.

1955 Lenticulina quenstedti (Gümbel). Seibold and Seibold, p. 105, pl. 13 , fig. 3 .

1962 Lenticulina plexus-quenstedti (Gümbel). Bastien and Sigal, p. 98, pl. 6 , figs. $15-20$, pl. 7 , figs. $1-3$.

1970 Lenticulina quenstedti (Gümbel). Winter, p. 26, pl. 3, fig. 92.

1971 Lenticulina quenstedti (Gümbel), forma A-C. Wernli, p. 322, pl. 4 , figs. $14,21,23,25,27-28$; pl. 10 , fig. 1 .

1972 Lenticulina quenstedti (Gümbel). Luterbacher, pl. 3, fig. 1.

1978 Lenticulina (Lenticulina) quenstedti (Gümbel). Munk, p. 43, pl. 3 , fig. 1.

1978 Lenticulina (Astacolus) volubilis Dain. Munk, p. 45, pl. 3, figs. 2, 9; pl. 4, figs. 7, 10 .

1978 Lenticulina (Lenticulina) sp. Munk, p. 43, pl. 3, fig. 7.

1978 Lenticulina quenstedti (Gümbel). Kuznetsova and Seibold, pl. 2, fig. 10.

?1980 Lenticulina ouachensis ouachensis (Sigal). Sliter, pl. 9, figs. 11-16; pl. 10, figs. 1-4.

Remarks. A Lenticulina with strong, very characteristic sutural ribs, fusing with a circular rib around the umbilical area. Toarcian to Tithonian (?Lower Cretaceous).

\section{Marginulina brunsvigensis Franke, 1936}

(PI. 1, Fig. 25)

+1936 Marginulina brunsvigensis Franke, p. 75, pl. 7, fig. 26.

1969 Vaginulina listi Bornemann. Brouwer, p. 40, pl. 3, fig. 24

\section{Marginulina dentaliniformis (Terquem and Berthelin, 1875)}

$$
\text { (Pl. 1, Fig. 26) }
$$

+1875 Cristellaria dentaliniformis Terquem and Berthelin, p. 43, pl. 3 , fig. 19.
1936 Marginulina simplex (Terquem). Franke, p. 75, pl. 7, figs. 23-24. 1937 Marginulina simplex (Terquem). Bartenstein and Brand, p. 159, pl. 1A, fig. 14; pl. 3, fig. 37; pl. 4, fig. 57.

\section{Marginulina oolithica (Terquem, 1870)}

$$
\text { (P1. 2, Fig. 78) }
$$

+1870 Dentalina oolithica Terquem (1870b), p. 28, figs. 5-15. 1941 Marginulina oolithica (Terquem). Frentzen, p. 336, pl. 3, figs. 26-29.

Marginulina porrecta Terquem, 1866

(Pl. 6, Fig. 161)

+1866 Marginulina porrecta Terquem, p. 501, pl. 21, figs. 19-21.

1936 Marginulina porrecta Terquem. Franke, p. 79, pl. 8, figs. 13-14. 1960 Marginulina porrecta Terquem. Bizon, p. 7, pl. 2, fig. 6; pl. 4, fig. 8.

Remarks. This elongate Marginulina with strong ribs is slightly asymmetrical. Sinemurian to upper Pliensbachian (Germany, France, England).

\section{Marginulina prima d'Orbigny, 1850}

(Pl. 6, figs. 162-163)

+1850 Marginulina prima d'Orbigny, p. 242.

1936 Marginulina prima d'Orbigny. Franke, pp. 76-78, pl. 8, figs. 1-7. 1936 Marginulina prima d'Orbigny. Macfadyen, p. 151, pl. 1, fig. 262. 1937 Marginulina prima d'Orbigny. Bartenstein and Brand, p. 161, pl. 2B, fig. 26; pl. 3, fig. 39; fig. 60; pl. 5, fig. 46.

1941 Marginulina prima d'Orbigny. Macfadyen, p. 38, pl. 2, fig. 32. 1957 Marginulina prima prima d'Orbigny. Nørvang, p. 367, figs. 98, 99, 103-104.

1968 Marginulina prima prima d’Orbigny. Welzel, p. 32, pl. 2, figs. $10-12$.

1969 Marginulina prima (d'Orbigny). Brouwer, p. 38, pl. 56, figs. 8, $10,13,14$, non figs. $9,11,12,15$.

Remarks. A common species in the Lias, but rather variable. Known from Hettangian to lowermost Toarcian (Germany, France, England, and Denmark).

\section{Marginulina spinata Terquem, 1858}

(Pl. 6, Fig. 160)

+1858 Marginulina spinata Terquem, p. 55 , pl. 3 , fig. 8 .

1936 Marginulina interrupta Terquem, f. spinata Terquem. Franke, p. 79 , pl. 8 , fig. 10 .

1937 Marginulina spinata spinata Terquem. Bartenstein and Brand, p. 161 , pl. 4 , fig. 61 ; pl. 5 , fig. 47 .

1941 Marginulina spinata Terquem. Macfadyen, p. 39, pl. 2, fig. 33. 1968 Marginulina prima spinata Terquem. Welzel, p. 33, pl. 2, fig. 22. Distribution: In Denmark, index-species for lower Pliensbachian. Occurs in Germany from lower Pliensbachian to lowermost Toarcian and in Britain ranges from the latest Sinemurian to early Toarcian (Copestake and Johnson, 1981).

\section{Marginulina variabilis Terquem. 1863}

$$
\text { (Pl. 6, Fig. 164) }
$$

+1863 Marginulina variabilis Terquem, p. 199, pl. 9, figs. 6-8.

1941 Marginulina lamellosa Terquem and Berthelin. Macfadyen, p. 37, pl. 2, fig. 30 .

Remarks. A rare, small Marginulina. Pliensbachian (France, England) and lowermost Toarcian (Southwest Germany).

Marginulinopsis baccularis (Terquem, 1866)

$$
\text { (PI. 7, Fig. 165) }
$$

+1866 Cristellaria baccularis Terquem, p. 514 , pl. 22, figs. 2-3.

\section{Marginulinopsis matutina matutina (d'Orbigny, 1850)}

(Pl. 7, Figs. 167-168)

+1850 Cristellaria matutina d'Orbigny, p. 242.

1936 Cristellaria matutina d'Orbigny. Macfadyen, p. 151, pl. 1, fig. 264. 1936 Cristellaria (Astacolus) antiquata d'Orbigny. Franke, p. 105, pl. 10 , fig. 14 .

1937 Cristellaria (Astacolus) vetusta d'Orbigny. Bartenstein and Brand, p. 172, pl. 6, fig. 31 . 
1937 Cristellaria (Astacolus) rectalonga Brand in Bartenstein and Brand, p. 172, pl. 4, fig. 80 .

\section{Marginulinopsis matutina terquemi (d'Orbigny, 1850)} (Pl. 7, Fig. 169)

+ 1850 Cristellaria terquemi d'Orbigny, p. 242.

1936 Cristellaria terquemi d'Orbigny. Macfadyen, p. 152, pl. 1, fig. 269.

1967 Marginulinopsis gr. vetusta (d'Orbigny), forma vetusta. Forme B. Ruget and Sigal, p. 36, pl. 2, fig. 1.

1967 Marginulinopsis sp. 1. Forme B. Ruget and Sigal, p. 37, pl. 1, fig. 6.

1967 Marginulinopsis gr. polygonata (Franke). Ruget and Sigal, p. 38, pl. 1, figs. 11-12.

1968 Astacolus primus d'Orbigny. Welzel, p. 44, pl. 2, fig. 42.

Marginulinopsis pauliniae (Terquem, 1866)

(PI. 7, Fig. 166)

+1866 Marginulina pauliniae Terquem, p. 427, pl. 17, fig. 5 . 1875 Marginulina constricta Terquem and Berthelin, p. 58, pl. 5, fig. 3. 1936 Marginulina simplex (Terquem). Franke, p. 75, pl. 7, fig. 22. 1937 Marginulina oolithica (Terquem). Bartenstein and Brand, p. 160, pl. 2A, fig. 11; pl. 2B, fig. 37; pl. 3, fig. 38.

Palmula anceps (Terquem, 1870) (Pl. 2, Fig. 81)

+1870 Cristellaria anceps Terquem, p. 428, pl. 9, figs. 11-21.

1932 Planularia semiinvoluta Terquem. Paalzow, p. 105, pl. 6, figs. $13-14,20-21$.

1962 Cristellaria plexus-treptensis Bastien and Sigal, p. 99, pl. 7, figs. 4-15.

\section{Palmula filosa (Terquem, 1866)}

(P1. 1, Fig. 29)

+1866 Cristellaria filosa Terquem, p. 517 , pl. 22 , fig. 8 .

1936 Cristellaria (Planularia) filosa Terquem. Franke, p. 95, pl. 9 fig. 25.

\section{Palmula gyrata (Terquem, 1870)}

(Pl. 2, Fig. 79)

+1870 Flabellina gyrata Terquem, p. 220, pl. 23, fig. 17.

Palmula hybrida (Terquem, 1870)

(Pl. 2. Fig. 80)

+1870 Flabellina hybrida Terquem (1870b), p. 229, pl. 24, fig. 16 . 1932 Flabellina deslongchampsi Terquem. Paalzow, p. 132, pl. 9, fig. 26.

1953 Falsopalmula deslongchampsi (Terquem). Seibold and Seibold, p. 58, pl. 4 , fig. 20 .

1960 Falsopalmual deslongchampsi (Terquem). Seibold and Seibold, p. 369 , text-fig. $6 \mathrm{k}-1$.

1960 Falsopalmula deslongchampsi (Terquem). Lutze, p. 464, pl. 32, fig. 15 .

\section{Palmula irregularis (Terquem 1862)}

(Pl. 1, Fig. 28)

+1862 Cristellaria irregularis Terquem, p. 445 , pl. 6 , fig. 2 .

1936 Cristellaria (Planularia) cordiformis Terquem. Franke, p. 95, pl.9, fig. 21.

\section{Palmula nucleata (Terquem, 1863)}

$$
\text { (Pl. 1, Fig. 27) }
$$

+1863 Cristellaria nucleata Terquem, p. 205, pl. 9, fig. 19.

1936 Cristellaria (Planularia) crepidula (Fichtel and Moll). Franke, p. 95 , pl. 9, fig. 22.

1957 Planularia pseudocrepidula Adams, p. 208, text-fig. 1-5.

1968 Planularia pseudocrepidula Adams. Welzel, p. 47, pl. 2, figs. 40-41.

Remarks. A variable and common, but small species. The cross section is flattened, the surface smooth. Sutures hardly visible. Equitant chambers can be observed in the adult stage of some specimens.
Palmula plebeia (Terquem and Berthelin, 1875)

(Pl. 1, Fig. 104)

+1875 Cristellaria plebeia Terquem and Berthelin, p. 44, pl. 3, fig. 22; pl. 4, fig. 1.

Palmula pikettyi (Terquem, 1866)

(Pl. 7, Fig. 171)

+1866 Cristellaria pikettyi Terquem, p. 511, pl. 21, figs. 31-32.

Palmula primaria (Franke, 1936)

(Pl. 1, Fig. 30)

+1936 Flabellina primaria Franke, p. 93, pl. 9, fig. 15.

Remarks. The type level given by Franke is of early Sinemurian age. The specimen of Fig. 30 differs from the holotype in having stronger ribs in the juvenile stage.

\section{Palmula primordialis (Terquem, 1870) \\ (Pl. 2, Fig. 82)}

+1870 Cristellaria primordialis Terquem (1870b), p. 428 , pl. 9, figs. $1-10$.

1962 Planularia sp. 13A. Bastien and Sigal, p. 102, pl. 7, fig. 18.

Palmula semiinvoluta (Terquem, 1870)

(Pl. 1, Fig. 26)

+1870 Cristellaria semi-involuta Terquem (1870b), p. 437, pl. 11, figs. $1-30$; pl. 12 , figs. $1-20 ; 25-38,30$; pl. 13 , figs. $1-3,4-13,15-$ 21,24 ; non pl. 12 , figs. $21-24$, 29; pl. 13 , figs. $3,14,22-23$.

\section{Palmula triquetra (Terquem, 1870)}

(Pl. 9, Fig. 210)

+1870 Flabellina triquetra Terquem (1870b), p. 225 , pl. 23, figs. 26-28.

1870 Flabellina semi-involuta Tertquem (1870b), p. 225, pl. 23, figs. 29-30; pl. 24, figs. 1-10.

Pseudonodosaria dubia (Terquem, 1870)

(Pl. 2, Fig. 83)

+ 1870 Glandulina dubia Terquem (1870b), p. 240, pl. 25, figs. 10-11.

Pseudonodosaria metensis (Terquem, 1862)

(Pl. 1, Fig. 33-34)

+1861 Glandulina metensis Terquem, p. 435, pl. 5, fig. 9 . 1866 Glandulina pygmaea Terquem, p. 478, pl. 19, fig. 6 .

Pseudonodosaria nitidana (Brand, 1937)

$$
\text { (Pl. 1, Fig. 36) }
$$

+1858 Nodosaria nitida Terquem, p. 30 , pl. 1, fig. 7 (preoccupied). 1862 Marginulina pupoides Terquem, p. 443, pl. 5, fig. 20.

1863 Dentalina strangulata Terquem, p. 172, pl. 7, fig. 7.

1866 Nodosaria primitiva Kübler and Zwingli, p. 7, pl. 1, fig. 16.

1870 Nodosaria primitiva Kübler and Zwingli, p. 5, pl. 1, fig. 1 .

1936 Nodosaria nitida Terquem. Franke, p. 44, pl. 3, fig. 22.

1937 Nodosaria nitidana Brand in Bartenstein and Brand, p. 143, pl. 2 B, fig. 22 ; pl. 4 , fig. 32 ; pl. 5 , fig. 27.

\section{Pseudonodosaria sexcostata (Bornemann, 1854)}

$$
\text { (Pl. 1, Fig. 32) }
$$

+1854 Glandulina sexcostata Bornemann, p. 32, pl. 2, fig. 7 . 1936 Glandulina sexcostata Bornemann. Franke, p. 58, pl. 6, fig. 2.

Remarks. The figured specimen is deformed by injury and therefore asymmetrical.

\section{Pseudonodosaria turbiniformis (Terquem, 1870) (PI. 2, Fig. 84)}

+1870 Glandulina turbiniformus Terquem (1870b), p. 240, pl. 25, fig. 9.

1941 Pseudoglandulina pupoides (Bornemann). Frentzen, p. 328, pl. 3 , fig. 5 . 
Pseudonodosaria vulgata (Bornemann, 1854)

(Pl. 1, Fig. 35)

+ 1854 Glandulina vulgata Bornemann, p. 31, pl. 2, figs. 1-2

1854 Glandulina tenuis Bornemann, p. 31, pl. 2, fig. 3.

1863 Glandulina oviformis Terquem, p. 168, pl. 7, fig. 4.

1936 Glandulina vulgata Bornemann. Franke, p. 54, pl. 5, fig. 9.

1936 Glandulina pygmaea Franke, p. 55, pl. 5, fig. 10.

1936 Glandulina tenuis Bornemann. Franke, p. 55, pl. 5, fig. 13.

\section{Saracenaria obesa (Terquem, 1866)}

(Pl. 1, Fig. 37)

+ 1866 Cristellaria obesa Terquem, p 436, pl. 18, fig. 3 .

1968 Saracenaria aff. hannoverana (Franke). Welzel, p. 49, pl. 2, fig. 50.

Remarks. A Saracenaria with rounded triangular cross section, without any keel and with strong chamber incisions. Seems to be restricted to the Pliensbachian and the lowermost Toarcian.

\section{Vaginulina anomala Blake, 1876}

(Pl. 1, Fig. 38)

+1876 Vaginulina anomala Blake in Tate and Blake, p. 464, pl. 17, fig 23.

1968 Citharina frankei Tappan. Welzel, p. 34, pl. 2, fig. 32.

Vaginulina contracta (Terquem, 1868)

(Pl. 7, Fig. 178)

+1868 Marginulina contracta Terquem, p. 125, pl. 8, figs. 13-24.

Vaginulina implicata (Schwager, 1865)

(Pl. 2, Fig. 85)

+1865 Cristellaria implicata Schwager, p. 127 , pl. 6, fig. 5 .

1932 Vaginulina flabellata Gümbel. Paalzow, p. 129, pl. 9, fig. 17.

1932 Vaginulina implicata Schwager. Paalzow, p. 129, pl. 9, fig. 19.

1954 Vaginulina zaglobensis var. parallela Bielecka and Poźaryski, p. 45 , pl. 6 , fig. 27.

1956 Citharina implicata (Schwager). Seibold and Seibold, p. 141, text-fig. 5i, k (1?); pl. 7, fig. 8

\section{Vaginulina aff. jurassica (Gümbel, 1862)}

(Pl.2, Fig. 86)

aff. 1862 Marginulina jurassica Gümbel, p. 222, pl. 3, fig. 21.

?1865 Cristellaria parallela Schwager, p. 121, pl. 5, fig. 5 .

aff. 1955 Vaginulina jurassica (Gümbel). Seibold and Seibold, p. 120, pl. 13 , fig. 15 , text-fig. 5 d, e.

?1956 Lenticulina (Planularia) pseudoparallela Seibold and Seibold, p. 114 , text-fig. $3 \mathrm{~m}-\mathrm{n}$.

1960 Dentalina bicornis Terquem. Seibold and Seibold, p. 358, textfig. 6q.

Remarks. The specimen of Fig. 87 is more slender than the holotype described by Gümbel (1862).

\section{Vaginulina parva Franke, 1936}

(Pl. 1, Figs. 39-40)

+1936 Vaginulina parva Franke, p. 83, pl. 8, fig. 23.

1936 Vaginulina constricta (Terquem and Berthelin). Franke, p. 83, pl. 8 , fig. 24 .

1937 Vaginulina constricta (Terquem and Berthelin). Bartenstein and Brand, p. 163 , pl. 1 A, fig. 16 ; pl. 2 B, fig. 28 ; pl. 3 , fig. 23 ; pl. 4 , fig. 65 .

Vaginulina perfoliata (Kübler and Zwingli, 1866) (PI. 1, Fig. 42)

+1866 Dentalina perfoliata Kübler and Zwingli, p. 8, pl. 1, fig. 11 . 1870 Dentalina perfoliata Kübler and Zwingli, p. 6, pl. 1, fig. 6 .

\section{Vaginulina triangula Frentzen, 1941}

(Pl. 1, Fig. 41)

+1941 Vaginulina triangula Frentzen, p. 340, pl. 4, fig. 8 .

Distribution. Found only in the upper Pliesbachian and lowermost Toarcian of Southwest Germany.
Vaginulina sp.

(PI. 2, Fig. 87)

1890 Cristellaria pauperata Parker and Jones. Haeusler, p. 109, pl. 14, fig. 53.

Lingulina acuformis (Terquem, 1866)

(Pl. 7, Fig. 179)

+1866 Frondicularia acuformis Terquem, p. 479 , pl. 19, fig. 8 .

1937 Frondicularia tenera prismatica Brand in Bartenstein and Brand, p. 156 , pl. 3 , fig. 34 , text-fig. 15 .

Remarks. A very slender, only slightly depressed Lingulina with 6 strong ribs and a subrectangular cross section. Found only in the upper Sinemurian and lower Pliensbachian of France and Germany. A good index-species.

\section{Lingulina affinis (Terquem, 1870)}

(Pl. 9, Fig. 217)

+ 1870 Dentalina affinis Terquem (1870b), p. 261, pl. 27, figs. 17-22. 1870 Frondicularia pyrus Kübler and Zwingli, p. 24, pl. 3, fig. 4.

\section{Lingulina costata (Kübler and Zwingli, 1866)}

(Pl. 1, Fig. 47; pl. 7, Fig. 172)

+ 1866 Frondicularia costata Kübler and Zwingli, p. 8, pl. 1, fig. 1 . 1870 Frondicularia longiscata Terquem (1870b), p. 216, pl. 22, figs. 23-24.

1870 Frondicularia nodosaria Terquem (1870b), p. 217; pl. 22, fig. 25-30.

1870 Frondicularia costata Kübler and Zwingli, p. 6, pl. 1, fig. 11 .

1884 Frondicularia inaequalis Deecke, p. 27, pl. 1, fig. 24.

1886 Frondicularia spatulata Terquem, p. 42, pl. 10, figs. 31-32.

1937 Frondicularia nodosaria Terquem. Bartenstein and Brand, p. 155 , pl. $12 \mathrm{~A}$, fig. 6 ; pl. 13, fig. 14 ; pl. $15 \mathrm{~A}$, fig. 21 .

1941 Lingularia nodosaria Terquem. Frentzen, p. 332, pl. 3, figs. 15-17.

1956 Lingularia nodosaria (Terquem). Barnard, p. 274, pl. 1, figs. 7-8.

1957 Lingularia longiscata var. alpha Adams, p. 224, text-figs. 116, 25-26.

1960 Frondicularia nodosaria (Terquem). Lutze, p. 468, pl. 32, fig. 13. 1968 Lingulina nodosaria Terquem. Welzel, p. 36, pl. 3, fig. 41.

1968 Frondicularia hauffi Franke. Welzel, p. 41, pl. 3, fig. 40.

1971 Lingulina nodosaria (Terquem). Wernli, p. 326, pl. 6, fig. 1-8.

Distribution. In many varieties from Sinemurian to Callovian.

Lingulina dentaliniformis (Terquem, 1870)

(Pl. 1, Fig. 48; Pl. 9, Fig. 216)

+1870 Frondicularia dentaliniformis Terquem (1870b), p. 217, pl. 23, figs. 1-8.

1870 Lingulina dentaliniformis Terquem (1870b), p. 237, pl. 225, figs. 1-3 (obj.)

1936 Lingulina laevissima (Terquem). Franke, p. 62, pl. 6, fig. 11.

1971 Lingulina dentaliniformis (Terquem). Wernli, p. 327, pl. 6, figs. 12-16.

\section{Lingulina cf. dentaliniformis (Terquem, 1870)}

(Pl. 9, Fig. 220)

Remarks. Differs from the latter species in having more constricted, more rounded chambers.

\section{Lingulina esseyana Deecke, 1886}

(Pl. 1, Fig. 49)

+1875 Lingulina ovalis Terquem and Berthelin, p. 23, pl. 1, fig. 27 (preoccupied).

1886 Lingulina esseyana Deecke, p. 312.

1936 Lingulina ovalis Terquem. Franke, p. 63, pl. 6, fig. 13.

1956 Lingulina esseyana Deecke. Barnard, p. 271, pl. 1, figs. 3-4.

1968 Lingulina esseyana Deecke. Welzel, p. 35, pl. 2, fig. 18.

\section{Lingulina franconica (Gümbel, 1862)}

(Pl. 9, Figs. 218-219)

+ 1862 Frondicularia franconica Gümbel, p. 219, pl. 3, fig. 13 . 1870 Frondicularia oolithica Terquem, p. 213, pl. 22, figs. 1-9. 
1890 Lingulina carinata d'Orbigny. Haeusler, p. 104, pl. 14, figs. 27-34.

1955 Lingulina franconica (Gümbel). Seibold and Seibold, p. 119, text-fig. 3e.

1965 Lingulina nodosaria Reuss. Gordon, p. 851, text-fig. 7/32-34.

1970 Frondicularia franconica franconica Gümbel. Winter, p. 24, pl. 3 , fig. 87.

Distribution. The species is reported from the upper Middle Jurassic to lower Kimmeridgian of France and South Germany.

\section{Lingulina gottingensis Franke, 1936}

(Pl. 1, Fig. 45)

+1936 Lingulina gottingensis Franke, p. 62, pl. 6, figs. 14-15.

Distribution. Ranges from the early Pliensbachian to earliest Toarcian (Copestake and Johnson, 1981).

\section{Lingulina laevissima (Terquem, 1866)}

(Pl. 1, Fig. 50)

+1866 Frondicularia laevissima Terquem, p. 481, pl. 19, fig. 19 . 1956 Lingulina laevissima (Terquem). Barnard, p. 272, pl, 1, fig. 5. 1968 Lingulina cernua (Berthelin). Welzel, p. 34, pl. 2, fig. 16. 1970 Lingulina sp. Mouterde and Ruget, p. 95, pl. 4, figs. 21-22.

Lingulina lingula (Terquem, 1862)

(Pl. 1, Fig. 44)

+1862 Frondicularia lingula Terquem, p. 437 , pl. 5 , fig. 8 . 1866 Frondicularia excavata Terquem, p. 403, pl. 15, fig. 4.

Lingulina nodosaria (Kübler and Zwingli, 1866)

$$
\text { (Pl. 9, Fig. 213) }
$$

+1866 Frondicularia nodosaria Kübler and Zwingli, p. 10, pl. 2, fig. 1 . 1870 Frondicularia nodosaria Kübler and Zwingli, p. 10, pl. 1, fig. 2.

\section{Lingulina taenoides Franke, 1936}

$$
\text { (Pl. 1, Fig. 43) }
$$

+1936 Lingulina taenoides Franke, p. 62, pl. 6, fig. 12.

\section{Lingulina tenera tenera Bornemann, 1854} (Pl. 7, Figs. 174-175)

+ 1854 Lingulina tenera Bornemann, p. 38, pl. 3, fig. 24 . 1936 Lingulina tenera Bornemann. Franke, p. 64, pl. 6, fig. 18 1956 Lingulina tenera Bornemann. Barnard, p. 274, pl. 1, figs. 1-2, 9-10.

1968 Lingulina tenera Bornemann. Welzel, p. 36, pl. 2, fig. 19.

Remarks. A variable species but one of the most characteristic species of the Lower Jurassic worldwide. Ranges from Hettangain to lowermost Toarcian. Reports from the Middle and Upper Jurassic are probably misidentified.

\section{Lingulina tenera carinata (Norvang, 1957)}

(Pl. 7, Fig. 180)

+1957 Geinitzina tenera carinata Nørvang, p. 62, figs. 46-55.

Distribution. Upper Sinemurian to lower Pliensbachian (Germany, Denmark).

\section{Lingulina tenera praepupa (Norvang, 1957)}

$$
\text { (Pl. 7, Fig. 173) }
$$

+1957 Geinitzina tenera praepupa Nørvang, p. 60; figs. 30-31.

Distribution. Upper Sinemurian to lower Pliensbachian of Europe.

\section{Lingulina tenera tenuistriata (Norvang, 1957)}

$$
\text { (PI. 7, Fig. 176) }
$$

+1957 Geinitzina tenera tenuistriata Nørvang, p. 56, figs. 13, 16, 17, 24.

Distribution. Hettangian to upper Sinemurian of Europe.

\section{Lingulina aff. tenera Bornemann, 1854}

$$
\text { (Pl. 1, Fig. 46) }
$$

Remarks. The ribs start as in Lingulina tenera tenera Bornemann, but end in the juvenile stage. Only chamber incisions are visible in the following stage. An identical form has been found in the early Toarcian (Tenuicostatum Zone, semicelatum Subzone) of Lincoln, eastern England (Copestake, pers. comm., 1984).

\section{Lingulina sp. \\ (Pl. 9, Fig. 212)}

Remarks. A small Lingulina, similar to Lingulina dentaliniformis (Terquem).

\section{Eoguttulina bilocularis (Terquem, 1864)}

(Pl. 2, Fig. 88)

+1864 Polymorphina bilocularis Terquem, p. 293, pl. 11, figs. 9-32. 1865 Globulina laevis Schwager, p. 137, pl. 7, fig. 6; non Figs. 5, 7.

1956 Eoguttulina bilocularis (Terquem). Seibold and Seibold, p. 143, text-fig. $6 \mathrm{c}$.

1962 Eoguttulina oolithica (Terquem). Cordey, p. 392, pl. 48, fig. 36.

Remarks. This Eoguttulina has a rounded cross section and a distinct subdivision between the final two chambers.

\section{Eoguttulina laevis (Schwager, 1865)}

$$
\text { (Pl. 2, Fig. 89) }
$$

+1865 Globulina laevis Schwager, p. 137, pl. 7, fig. 7; non Figs. 5-6.

Remarks. The two visible chambers are separated by a deep incision. Cross section ovate.

\section{Eoguttulina liassica (Strickland, 1846)}

(Pl. 1, Fig. 51)

+1846 Polymorphina liassica Strickland, p. 31, text-fig. b.

Remarks. Outline ovate, cross section compressed, incision weak.

\section{Eoguttulina metensis (Terquem, 1864)}

(Pl. 1, Fig. 53; Pl. 2, Fig. 90)

+1864 Polymorphina metensis Terquem, p. 301, pl. 13, fig. 38 . 1865 Globulina laevis Schwager, p. 137, pl. 7, fig. 5; non Figs. 6-7. 1968 Eoguttulina liassica liassica (Strickland). Welzel, p. 51, pl. 3, fig. 36 .

Remarks. An elongated, nearly rounded Eoguttulina without incisions.

\section{Eoguttulina oolithica (Terquem, 1876)}

$$
\text { (Pl. 1, Fig. 54) }
$$

+1876 Globulina oolithica Terquem, p. 497, pl. 17, fig. 12 .

Remarks. A short Eoguttulina, nearly circular in cross section, no incisions.

\section{Eoguttulina subrhomboidalis (Schwager, 1865)}

(Pl. 9, Fig. 214)

+1865 Vulvulina subrhomboidalis Schwager, p. 140, pl. 7, fig. 17 . 1956 Guttulina subrhomboidalis (Schwager). Seibold and Seibold, p. 148 , text-fig. $6 \mathrm{k}$.

1960 Palaeopolymorphina sp. E. and I. Seibold, p. 370, text-fig. 7c.

Distribution. Known only from the Oxfordian of south Germany.

\section{Eoguttulina ventricosa (Terquem, 1864)}

$$
\text { (P1. 1, Fig. 52) }
$$

+1864 Polymorphina ventricosa Terquem, p. 302, pl. 13, fig. 42 . 1936 Polymorphina kuhni Franke, p. 120, pl. 12, fig. 7.

Remarks. A flattened Eoguttulina with weak incisions or without visible sutures and an ovate outline.

\section{Ramulina ef. spandelia Paalzow, 1917}

(Pl. 9, Fig. 215)

1890 Lagena hispida Reuss. Haeusler, p. 88, pl. 123, figs. 21-24.

1890 Lagena aspera Reuss. Haeusler, p. 89, pl. 13, figs. 25-26.

1890 Nodosaria hispida d'Orbigny. Haeusler, p. 103, pl. 15, fig. 40.

cf. 1917 Ramulina spandeli Paalzow, p. 246, pl. 47, fig. 15.

1960 Ramulina spandeli Paalzow. Seibold and Seibold, p. 372, text-

fig. 71.

1962 Ramulina cf. spandeli Paalzow. Cordey, p. 392, pl. 47, fig. 31 1970 Ramulina fusiformis Khan. Winter, p. 41, pl. 4, fig. 140.

1972 Ramulina nodosarioides Deain. Luterbacher, pl. 2, fig. 21 
Remarks. Fig. 215 differs from the holotype in having more inflated chambers.

\section{Brizalina liasica (Terquem, 1858)}

(Pl. 1, Figs. 19-20)

+1858 Textularia liasica Terquem, p. 75, pl. 4, fig. 12 .

1858 Textularia metensis Terquem, p. 75, pl. 4, fig. 13.

1870 Textularia prodromus Kübler and Zwingli, p. 7, pl. 1, fig. 17.

1875 Textularia limbata Terquem and Berthelin, p. 63, pl. 5, fig. 9.

1875 Textularia vicinalis Terquem and Berthelin, p. 63, pl. 5, fig. 10.

1936 Textularia pikettyi Terquem. Franke, p. 125, pl. 12, fig. 19.

1936 Bolivina rhumbleri Franke, p. 126, pl. 12, fig. 21.

1937 Bolivina rhumbleri rhumbleri Franke. Bartenstein and Brand, p. 184 , pl. 4 , fig. 73 ; pl. 5 , fig. 72 .

1937 Bolivina rhumbleri amalthea Brand in Bartenstein and Brand, p. 185 , pl. 7 , fig. 1 .

1957 Bolivina liasica (Terquem). Nørvang, p. 387, fig. 182.

1960 Bolivina liasica (Terquem). Bizon, p. 14, pl. 3, fig. 7; pl. 4, fig. 6 . 1965 Bolivina liasica liasica (Terquem). Witthuhn, p. 69, pl. 1, figs. 3-4. 1965 Bolivina liasica amalthea Brand. Witthuhn, p. 73, pl. 1, figs. 5-6. 1968 Brizalina? liasica liasica (Terquem). Welzel, p. 52, pl. 3, fig. 43 1969 Brizalina liasica (Terquem). Brouwer, p. 41, pl. 7, fig. 20-27.

Distribution. Upper Sinemurian (Switzerland), lower Pliensbachian to lowermost Toarcian (Germany, France).

\section{Spirillina concava (Terquem, 1870)}

(Pl. 2, Fig. 91)

+1870 Cornuspira concava Terquem (1870b), p. 244 , pl. 25 , fig. 16

Remarks. More numerous and more slender whorls than Spirillina gracilis Terquem.

\section{Spirillina gracilis Terquem, 1886}

$$
\text { (Pl. 2, Fig. 92) }
$$

+1886 Spirillina gracilis Terquem, p. 8, pl. 1, fig. 12 .

\section{Spirillina numismalis Terquem and Berthelin, 1875}

(Pl. 7, Fig. 177)

+1875 Spirillina numismalis Terquem and Berthelin, p. 17, pl. 1, fig. 13.

1968 Spirillina polygyrate Gümbel. Welzel, p. 52, pl. 3, fig. 6.

\section{Trocholina umbo Frentzen, 1941}

(Pl. 7, Figs. 181-182)

+1941 Trocholina umbo Frentzen, p. 306, pl. 1, fig. 12.

?1941 Trocholina intermedia Frentzen, p. 305, pl. 1, fig. 10.

?1941 Ammodiscoides clypeiformis Frentzen, p. 302, pl. 1, fig. 6.

1952 Trocholina intermedia Frentzen. Wicher, p. 262.

Distribution. Known only from the Sinemurian of southwest Germany.

"Patellinella" poddari Subbotina and Srivastava, 1960

(Pl. 10, Figs. 222-224)

+1960 Patellinella poddari Subbotina and Srivastava in Subbotina et al., p. 43 , pl. 4 , fig. 7

?1960 Paalzowella feifeli seiboldi Lutze, p. 486, pl. 33, fig. 12.

1978 Patellinella poddari Subbotina and Srivastava. Bhalla and Ab-

bas, p. 188 , pl. 13, fig. 1-2.

Remarks. Described from the Callovian of India.

Epistomina sp.

(Pl. 2, Figs. 93-95; Pl. 9, Fig. 221)

Remarks. Asymmetrical trochoid tests with light constrictions on the margin of the dorsal side and flattened ventral side. The bad preservation does not allow a more precise determination. Ribs could not be observed.

Radiolarians (PI. 10, Figs. 225-234)

Remarks. All radiolarians are internal calcitic moulds and therefore very difficult to determine. Some species could be identified after Foreman (1978) (see Fig. 5).
Note on the Plates. The illustrated specimens are assigned numbers (5373-5410, 5506-5628, 5801-5900, and 6034) which correspond to negative and specimen I.D. numbers at the Geologisch-Paläontologisches Institut, Universität Tübingen, West Germany.

\section{ACKNOWLEDGMENTS}

The authors gratefully acknowledge the critical and thorough reviews of the manuscript by P. Copestake (Britoil), D. Eicher (University of Colorado), F. Gradstein (Geological Survey of Canada), W. Sliter (U.S. Geological Survey) and B. Stam (Dalhousie University). Discussions with L. Jansa and E. Winterer were most helpful. Acknowledgment is made to the donors of The Petroleum Research Fund, administered by the American Chemical Society for research support to R. M. Leckie. Typing of the final manuscript by P. Leckie is greatly appreciated.

\section{REFERENCES}

Adams, G. C., 1957. A study of the morphology and variation of some Upper Lias foraminifera. Micropaleontology, 3:205-226.

Ager, D. V., 1974. The western High Atlas of Morocco and their significance in the history of the North Atlantic. Proc. Geol. Assoc., $85: 23-41$.

Barbieri, F., 1964. Micropaleontologia del Lias e Dogger del Pozzo Ragusa 1 (Sicilia). Riv. Ital. Palent., 70:709-831.

Barnard, T., 1956. Some Lingulinae from the Lias of England. Micropaleontology, 2:271-282.

1957. Frondicularia from the Lower Lias of England. Micropaleontology, 3:171-181. 1959. Some arenaceous foraminifera from the Lias of England. Contr. Cushman Found. Foram. Res., 10:132-136.

Bartenstein, H., 1974. Upper Jurassic-Lower Cretaceous primitive arenaceous foraminifera from DSDP Sites 259 and 261, eastern Indian Ocean. In Veevers, J. J., Heirzler, J. R., et al., Init. Repts. DSDP, 27: Washington (U.S. Govt. Printing Office), 683-695.

Bartenstein, H., and Brand, E., 1937. Mikro-paläontologische Untersuchungen zur Stratigraphie des nordwest-deutschen Lias und Doggers. Abh. Senckenb. Naturforsch. Ges., 439:1-224.

Bastien, M.-Th., and Sigal, J., 1962. Les foraminifères. In Enay, R. (Ed.), Contribution a l'étude paléontologique de l'Oxfordien supérieur de Trept (Isère). Trav. Lab. Geol. Fac. Sci. Lyon, n. s., 8: 83-123.

Berthelin, G., 1879. Foraminiféres du Lias moyen de la Vendée. Rev. Mag. Zool, Pure, Ser. 3, 7:24-41.

Bessler, J., 1938. Paläontologische Notizen aus den Badischen Landessammlungen für Naturkunde, Karlsruhe i. B. Funde von Hadrocheilus Till im oberen Lias Gamma (Davoei-Schichten) der Langenbrückener Senke. Beitr. Naturkdl. Forsch. Südwestdeutschl., 3: 66-75.

Bhalla, S. N., and Abbas, S. M., 1978. Jurassic foraminifera from Kutch, India. Micropaleontology, 24:160-209.

Bielecka, W., and Poźaryski, W., 1954. Micropalaeontological stratigraphy of the Upper Malm in central Poland. Inst. Geol. Prace, 12: $1-206$.

Bizon, G., 1960. Révision de quelques espèces-types de Foraminiferères du Lias du Bassin Parisien de la collection Terquem. Rev. Micropaléont., 3:3-18.

Bornemann, J. G., (Sr.), 1854. Über die Liasformation in der Umgegend von Göttingen [Thesis]. Univ. of Berlin.

Brouwer, J., 1969. Foraminiferal assemblages from the Lias of NorthWestern Europe. Verh. K. Nederl. Akad. Wetensch., Ser. Natuurkd., 25:1-48.

Burbach, O., 1886a. Beiträge zur Kenntnis der Foraminiferen des mittleren Lias vom grossen Seeberg bei Gotha. 1. Die Gattung Frondicularia Defr. Corr.-Bl. Naturwiss. Ver., 1886:1-53; Z. Naturwiss., 59:30-53.

1886b. Beiträge zur Kenntnis der Foraminiferen des mittleren Lias vom grossen Seeberg bei Gotha. 2. Die Milioliden. $Z$. Naturwiss., 59:490-502.

Copestake, P., and Johnson, B., 1981. Jurassic, Part I. The Hettangian to Toarcian. In Jenkins, D. G., and Murray, J. W. (Eds.), Stratigraphical Atlas of Fossil Foraminifera: Chichester (Ellis Horwood), pp. 81-105. 
, in press. Lower Jurassic (Hettangian-Toarcian) foraminifera from the Mochras Borehole, North Wales (U.K.); biostratigraphic and systematic micropalaeontology. Inst. Geol. Sci. Rept.

Cordey, W. G., 1962. Foraminifera from the Oxford Clay of Staffin Bay, Isle of Skye, Scotland. Senckenb. Leth., 43:375-409.

Croneis, C., and McCormack, J., 1932. Fossil Holothuroidea. J. Paleontol., 6:111-148.

Dain, L. G., 1972. Foraminifera from Upper Jurassic deposits of western Siberia. Trudy Vsesoj. Neftj. Nauchno-Issled. Geol. Inst., 317: 1-271. (In Russian)

Deecke, W., 1884. Die Foraminiferenfauna der Zone des Stephanoceras humphriesianum im Unter-Elsass. Abh. Geol. Spez.-Karte Els.-Lothr., 4:1-68.

1886. Les foraminifères de l'Oxfordien des environs des Montbéliard (Doubs). In Kilian, W. (Ed.), Notes Géologiques sur le Jura du Doubs. Mém. Soc. Émul., 16:289-335.

Exton, J., and Gradstein, F. M., in press. Early Jurassic Stratigraphy and Micropaleontology of the Grand Banks and Portugal. 3rd North Amer. Paleontol. Convention, Montreal, 1982: Geol. Assoc. Canada Mem.

Foreman, H. P., 1978. Mesozoic Radiolaria in the Atlantic Ocean off the northwest coast of Africa, Deep Sea Drilling Project, Leg 41 In Lancelot, Y., Seibold, E., et al., Init. Repts. DSDP, 41: Washington (U.S. Govt. Printing Office), 739-761.

Franke, A., 1936. Die Foraminiferen des deutschen Lias. Abh. Preuss. Geol. Ladesanst., n.s., 169:1-138.

Frentzen, K., 1941. Die Foraminiferenfauna des Lias, Dogger und unteren Malm der Umgegend von Blumberg (Oberes Wutachgebiet). Beitr. Naturkdl. Forsch. Oberrh., 6:125-402.

1944. Die agglutinierenden Foraminiferen der Birmenstorfer Schichten (Transverarius-Zone in Schwammfazies) des Gebietes um Blumberg in Baden. Paläont. Z., 23:317-348.

1964. Funde von Holothurien-Laklkörperchen im Jura des Oberrheingebietes. Beitr. Naturkundl. Forsch. SW-Deutschland, 23: 31-51.

Frizzell, D. L., and Exline, H., 1955. Monograph of Fossil Holothurian Sclerites. Bull. Univ. Missouri Sch. Mines Met., Techn. Ser., 89.

Fuchs, W., 1970. Eine alpine, tiefliassische Foraminiferenfauna von Hernstein in Niederösterreich. Verh. Geol. Bundesanst. Wien, pp. 66-145.

Gerke, A. A., 1961. Foraminifera from Permian, Triassic and Liassic deposits of the oil-fields in Northern Siberia. Trudy Nauchno-Issled. Inst. Geol. Arktiki, 120:1-518. (In Russian)

Gordon, W. A., 1961. Some foraminifera from the Ampthill Clay, Upper Jurassic, of Cambridgeshire. Palaeontology, 4:520-537. 1965. Foraminifera from the Corallian beds, Upper Jurassic, of Dorset, England. J. Paleont., 39:828-863.

1970. Biogeography of Jurassic Foraminifera. Geol. Soc. Am. Bull., 81:1689-1704.

Gradstein, F. M., 1976. Biostratigraphy and biogeography of Jurassic Grand Banks Foraminifera. Proc. "Benthonics '75", Halifax, Nova Scotia. Maritime Sediments Spec. Publ., 1(Pt. B):557-583.

, 1978. Jurassic Grand Banks Foraminifera. J. Foram. Res., 8:971-109.

1983. Paleoecology and stratigraphy of Jurassic abyssal Foraminifera in the Blake-Bahama Basin, Deep Sea Drilling Project Site 534. In Sheridan, R. S., Gradstein, F. M., et al., Init. Repts. DSDP, 76: Washington (U.S. Govt. Printing Office), 537-560.

Gradstein, F. M., Williams, G. L., Jenkins, W. A. M., and Ascoli, P., 1975. Mesozoic and Cenozoic stratigraphy of the Atlantic continental margin, Eastern Canada. In Yorath, C. J., Parker, E. R., and Glass, D. J. (Eds.), Canada's Continental Margins and Offshore Petroleum Exploration. Can. Soc. Petrol. Geol. Mem., 4: 103-131.

Groiss, J. Th., 1967. Foraminiferen-Faunen aus den Neuburger Bankkalken (Mittel-Tithon). Erlanger Geol. Abh., 66:1-74.

Gümbel, C., 1862. Die Streitberger Schwammlager und ihre Foraminiferen-Einschlüsse. Jh. Ver. Vaterl. Naturkd. Württemb., 18:192-238.

Haeusler, R., 1881. Untersuchungen über die mikroskopischen Strukturverhältnisse der Aargauer Jurakalke mit besonderer Berücksichtigung ihrer Foraminiferenfauna [Thesis]. Univ. of Zürich.

1882. Notes on the Trochamminae of the Lower Malm of the Canton Aargau (Switzerland). Ann. Mag. Nat. Hist., Ser. 5, 10:49-61.
1886. Die Lituolidenfauna der Aargau'schen Impressaschichten. N. Jb. Mineral. Geol. Palaeont., 4:1-30.

1890. Monographie der Foraminiferen-Fauna der schweizerischen transversarius-Zone. Abh. Schweiz. Palaont. Ges., 17: $1-134$.

Hohenegger, J., 1981. Ichthyolaria densicostata n. sp., eine charakteristische Foraminifere des Unteren Lias Mitteleuropas. Stuttgarter Beitr. Naturkd., Ser. B, 74:1-33.

Issler, A., 1908. Beiträge zur Stratigraphie und Mikrofauna des Lias in Schwaben. Palaeontographica, 55:1-104.

Jansa, L. F., and Wiedmann, J., 1982. Mesozoic-Cenozoic development of the eastern North American and northwest African continental margins: a comparison. In von Rad, U., Hinz, K., Sarnthein, M., and Seibold, E. (Eds.), Geology of the Northwest African Continental Margin: Berlin (Springer-Verlag), pp. 215-269.

Jendryka-Fuglewicz, B., 1975. Evolution of the Jurassic and Cretaceous smooth-walled Lenticulina (Foraminifera) of Poland. Acta Paleont. Pol., 20:99-197.

Klähn, H., 1924. Die Foraminiferen des elsässischen Giganteustones, unter besonderer Berücksichtigung der oberelsässischen Vorkommnisse. Jb. Preuss. Geol. Landesanst., 44(1923):449-464.

Kübler, J., and Zwingli, H., 1866. Microskopische Bilder aus der Urwelt der Schweiz. Neujahrsbl. Bürgerbibliothek (Vol. 2). Winterthur (Steiner). (Steiner).

Kuznetsova, K. I., 1974. Distribution of benthonic foraminifera in Upper Jurassic and Lower Cretaceous deposits at Site 261, DSDP Leg 27, in the eastern Indian Ocean. In Veevers, J. J., Heirzler, J. R., et al., Init. Repts. DSDP, 27: Washington (U.S. Govt. Printing Office), 673-682.

Kuznetsova, K. I., and Seibold, I., 1978. Foraminifers from the Upper Jurassic and Lower Cretaceous of the eastern Atlantic (DSDP Leg 41, Sites 367 and 370). In Lancelot, Y., Seibold, E., et al., Init. Repts. DSDP, 41: Washington (U.S. Govt. Printing Office), 515-537.

Lancelot, Y., and Winterer, E. L., 1980. Evolution of the Moroccan Oceanic Basin and adjacent continental margin-A Synthesis. In Lancelot, Y., Winterer, E. L., et al., Init. Repts. DSDP, 50: Washington (U.S. Govt. Printing Office), 801-821.

Lloyd, A. J., 1959. Arenaceous foraminifera from the type Kimmeridgian (Upper Jurassic). Palaeontology, 1:298-320.

Loeblich, A. R., Jr., and Tappan, H., 1946. New Washita foraminifera. J. Paleont., 20:238-258.

1950. North American Jurassic foraminifera. 1. The type Redwater Shale (Oxfordian) of South Dakota. J. Paleont., 24:39-60.

Luterbacher, H., 1972. Foraminifera from the Lower Cretaceous and Upper Jurassic of the northwestern Atlantic OCean. In Hollister, C. D., Ewing, J. I., et al., Init. Repts. DSDP, 11: Washington (U.S. Govt. Printing Office), 561-594.

Lutze, G. F., 1960. Zur Stratigraphie und Paläontologie des Callovien und Oxfordien in Nordwest-Deutschland. Geol. Jb., 77:391-532.

Macfadyen, W. A., 1936. d'Orbigny's Lias Foraminifera. J. R. Microsc. Soc., 56:147-153.

, 1941. Foraminifera from the green ammonite beds, Lower Lias, of Dorset. Phil. Trans. R. Soc. London, Ser. B, 231:1-73.

Mouterde, R., and Ruget, C., 1970. Le Lias moyen de Sao Pedro de Muel (Deuxième partie: paléontologie). II. Les Foraminifères. Comunic. Serv Geol. Port., 54:79-108.

Müller-Stoll, H., 1936. Beiträge zur Anatomie der Belemnoidea. $N$. Acta Leopoldina, N. S., 4:159-226.

Munk, Ch., 1978. Feinstratigraphische und micropalaontologische Untersuchungen an Foraminiferen-faunen im Mittleren und Oberen Dogger (Bajocien-Callovien) der Frankenalb. Erlanger Geol. Abh., 105:1-72.

Nørvang, A., 1957. The foraminifera of the Lias Series in Jutland. Denmark Medd. Dansk Geol. Foren., 13:278-413.

Oesterle, H., 1968. Foraminiferen der Typlokalitat der BirmenstorferSchichten, unterer Malm (Teilrevision der Arbeiten von J. Kübler und H. Zwingli 1866-1870 und von R. Haeusler 1881-1893). Eclogae Geol. Helv., 61:695-792.

Oppel, A., 1866. Über die Zone des Ammonites transversarius. Geogn.Paläont. Beitr., 1:205-318. 
Orbigny, A., d', 1850. Prodrôme de paléontologie stratigraphique universelle des animaux mollusques et rayonnés: Paris (Masson).

Paalzow, R., 1917. Beiträge zur Kenntnis der Foraminiferenfauna der Schwamm-Mergel des Unteren Weissen Jura in Süddeutschland. Abh. Naturhist. Ges. Nürnberg, 19:203-248. 1922. Die Foraminiferen der parkinsoni-Mergel von Heidenheim am Hahnenkamm. Abh. Naturhist. Ges. Nürnberg, 22: $1-35$.

1932. Die Foraminiferen aus den Transversarius-Schichten und Impressa-Tonen der nordöstlichen Schwäbischen Alb. Jh. Ver. Vaterl. Naturkd. Württemb., 88:81-142.

Rabitz, G., 1963. Foraminiferen des Göttinger Lias. Palaont. Z., 37: 198-224.

Rioult, M., 1961. Les "sclérites d'holothuries fossiles du Lias". Colloque Sur Le Lias Francais. Mém. Bur. Rech. Géol. Min., 4: 121-153.

Ruget, C., and Sigal, J., 1967. Les foraminifères du sondage de Laneuveville-devant-Nancy (Lotharingien de la région type). Sci. Terre, 12:33-70.

Schwager, C., 1865. Beiträg zur Kenntnis der mikroskopischen Fauna jurassischer Schichten. Jh. Ver. Vaterl. Naturkd. Württemb., 21: 82-151.

Seibold, E., and Seibold, I., 1953. Foraminiferenfauna und Kalkgehalt eines Profils im gebankten unteren Malm Schwabens. $N$. Jb. Geol. Paläont. Abh., 98:28-86.

1955. Revision der Foraminiferen-Bearbeitung C. W. Gümbels (1862) aus den Streitberger Schwamm-Mergeln (Oberfranken, Unterer Malm). N. Jb. Geol. Paläont. Abh., 101:91-134. 1956. Revision der Foraminiferen-Bearbeitung C. Schwagers (1865) aus den Impressa-Schichten (Unterer Malm) Süddeutschlands. N. Jb. Geol. Paläont. Abh., 103:91-154.

1960. Foraminiferen der Bank- und Schwamm-Fazies im unteren Malm Süddeutschlands. N. Jb. Geol. Paläont. Abh., 109: 309-438.

Shipp, D., and Murray, J. W., 1981. Jurassic, Part III. The Callovian to Portlandian. In Jenkins, D. G., and Murray, J. W. (Eds.), Stratigraphical Atlas of Fossil Foraminifera: Chichester (Ellis Horwood), pp. 125-144.

Sliter, W. V., 1980. Mesozoic foraminifers and deep-sea benthic environments from Deep Sea Drilling Project Sites 415 and 416, eastern North Atlantic. In Lancelot, Y., Winterer, E. L., et al., Init. Repts. DSDP, 50: Washington (U.S. Govt. Printing Office), 353-420.

Strickland, H. E., 1846. On two species of microscopic shells found in the Lias. Quart. J. Geol. Soc. London, 2:30-31.

Ströbel, W., 1944. Mikrofauna im Weissen Jura alpha der mittleren und Südwestalb. N. Jb. Mineral. Geol. Paläont. Abh., Ser. B, 88: 1-39.

Subbotina, N. N., Datta, A. K., and Srivastava, B. N., 1960. Foraminifera from the Upper Jurassic deposits of Rajastan (Jaisalmar) and Kutch, India. Bull. Geol. Min. Met. Soc. India, 23:1-48

Tappan, H., 1951. Northern Alaska index Foraminifera. Contr. Cushman Found. Res., 2:1-8.

1955. Foraminifera from the Arctic Slope of Alaska. Part 2, Jurassic Foraminifera. U.S. Geol. Surv. Prof. Paper, 236B:21-86.

Tate, R., and Blake, J. F., 1876. The Yorkshire Lias: London (John van Voorst).
Terquem, O., 1858. Recherches sur les foraminifères du Lias du Département de la Moselle. Mém. Acad. Imp. Metz, Ser. 2, 39: 563-656.

1860-61. Recherches sur les foraminifères de l'étage moyen et de l'étage inferieur du Lias. Mém. Acad. Imp. Metz, 42:415-466. 1862-63. Troisième mémoire sur les foraminifères du Lias des Départements de la Moselle, de la Côte-d'Or, du Rhône, de la Vienne, et du Calvados. Mém. Acad. Imp. Metz, 44:361-438.

1864. Quatrième mémoire sur les foraminifères du Lias comprenant les Polymorphines des Départements de la Moselle, de la Côte-d'Or et de l'Indre: Metz (Lorette). 1866a. Cinquième mémoire sur les foraminifères du Lias des Départements de la Mosella, de la Côte-d'Or et de l'Indre: Metz (Lorette).

, 1866b. Sixième mémoire sur les foraminifères du Lias des Départements de l'Indre et de la Moselle: Metz (Lorette). 1868. Premier mémoire sur les foraminifères du système oolithique. Bull. Soc. Hist. Nat. Departem. Moselle, 11:1-138. $1870 \mathrm{a}$. Deuxième mémoire sur les foraminifères du système oolithique: Monographie des Cristellaires de la zone à Ammonites parkinsoni de Fontoy (Moselle). Mem. Acad. Imp. Metz, 50(186869):403-456.

$1870 \mathrm{~b}$. Mémoires sur les Foraminifères du système Oolithique. Troisiême mémoire sur les Foraminifères du système Oolithique comprenant les genres Frondicularia, Flabellina, Nodosaria, Dentalina, etc., de la zone à Ammonites parkinsoni de Fontoy (Moselle): Metz (Lorette).

1876. Recherches sur les foraminifères du Bajocien de la Moselle. Bull. Soc. Géol. France, Ser. 3, 4:477-501.

1886. Les foraminifères et les ostracodes du Fuller's-earth des environs de Varsovie. Mém. Soc. Géol. France, Ser. 3, 4:1-112.

Terquem, O., and Berthelin, G., 1875. Étude microscopique des marnes du Lias Moyen d'Essey-les-Nancy, zone inférieure de l'Assise à Ammonites margaritatus. Mém. Soc. Géol. France, Ser. 2, 10: $1-126$.

Till, A., 1907. Die fossilen Cephalopodengebisse. Jb. Kais. K. Geol. Reichsanst., 57:535-682.

Welzel, E., 1968. Foraminiferen und Fazies des fränkischen Domeriums. Erlanger Geol. Abh., 69:1-79.

Wernli, R., 1971. Les foraminifères du Dogger méridional (France). Arch. Sci., 24:305-364.

Wicher, C. A., 1952. Involutina, Trocholina und Vidalina -Fossilien des Riffbereichs. Geol. Jb., 66:257-284.

Winter, B., 1970. Foraminiferenfaunen des Unter-Kimmeridge (Mittlerer Malm) in Franken. Erlanger Geol. Abh., 79:1-56.

Wiśniowski, T., 1890. Mikrofauna der Ornaten-Tone der Umgebung von Krakau. Teil. Foraminiferen des Oberen Calloviums von Grojec. Akad. Um. Mat. Przyr. Pam., 17:181-242.

Witthuhn, W., 1965. Zur Phylogenie von Bolivina (Foraminifera) aus dem Mittleren Lias Nordwestdeutschlands [Thesis]. Tech. Hochschule Braunschweig.

Ziegler, J. H., 1959. Mikropaläontologische Untersuchungen zur Stratigraphie des Braunjura in Nordbayern. Geologica Bavar., 40: $11-128$

Zittel, K. A., 1869. Geologische Beobachtungen aus den Central-Appenninen. Geogn.-Paläont. Beitr., 2:93-176.

Date of Initial Receipt: August 3, 1983

Date of Acceptance: February 3, 1984 
Plate 1. Jurassic ostracodes and Liassic foraminifers. (Specimens are from Sample 547B-11,CC and figures are magnified $\times 38$ unless otherwise indicated.) 1. Polycope cf. cerasia, 547B-18-2, 5-7 cm, No. 5379, $\times 50$. 2. Polycope pelta, No. $5409, \times 50$. 3-4.Ledahia septenaria, (3) No. 5397, $\times 50$, (4) No. 5398, $\times 50$. 5-6. Gen. et sp. indet., (5) 547B-18-2, 5-7 cm, No. 5375, ×50, (6) 547B-7-2, 35-36 cm, No 5802, $\times 50.7$. Lagenammina jurassica, No. $5516, \times 50$. 8. Ammodiscus glumaceus, No. $5519, \times 50$. 9. ?Tolypammina sp., 547B-20-2, 59-61 cm, No. 5528; view from below to the internal structure, $\times 50$. 10. Haplophragmoides kingakensis, 547B-18-2, 5-7 cm, No. 5525, $\times 100$. 11. Haplophragmoides barrowensis, 547B-20-2, 59-61 cm, No. 5524, $\times 50$. 12. Haplophragmium pictonicum, No. 5529 . 13. Ophthalmidium liasicum, No. 5532 , $\times 50$. 14. Ophthalmidium carinatum No. $5531, \times 50$. 15. Nodosaria caudata, No. $5534, \times 25$. 16. Dentalina teutoburgensis, 547B-20-1, 126-129 cm, No. 5559. 17. Dentalina gyrosa, No. 5548. 18. Astacolus semiincisus, No. 5565, $\times 38$. 19-20. Brizalina liasica, (19) No. 5568, (20) No. 5569, ×75. 21. Frondicularia major, No. 5608. 22. Frondicularia sacculus, No. 5613. 23. Lenticulina polygonata, No. 5577. 24. Marginulina brunsvigensis, 547B-22-2, 55-57 cm, No. 5585. 25. Marginulina dentaliniformis, 547B-17,CC, No. 5579. 26. Palmula semiinvoluta, No. 5591. 27. Palmula nucleata, 547B-18-2, 5-7 cm, No. 5594. 28. Palmula irregularis, No. 5604. 29. Palmula filosa, 547B-22-2, 55-57 cm, No. 5592. 30. Palmula primaria, No. 5593. 31. Palmula plebeia, No. 5566. 32. Pseudonodosaria sexcostata, misshapen. No. 5599. 33-34.Pseudonodosaria metensis, (33) 547B-20-1, 126-129 cm, No. 5597, (34) No. 5600 b. 35. Pseudonodosaria vulgata No. 5600 a. 36. Pseudonodosaria nitidana No. 5596. 37. Saracenaria obesa, No. 5620 b. 38. Vaginulina anomala, No. 5603 a. 39-40.Vaginulina parva, (39) $547 \mathrm{~B}-22-1,95-98 \mathrm{~cm}$, No. $5603 \mathrm{~b}$, (40) No. 5601 . 41. Vaginulina triangula, No. 5602 a. 42. Vaginulina perfoliata, 547B-18-2, 5-7 cm, No. 5602 b. 43. Lingulina taenioides, No. 5614. 44. Lingulina lingula, 547B-20-1, 126-129, No. 5615. 45. Lingulina gottingensis, No. 5607. 46. Lingulina aff. tenera Bornemann, No. 5620 a. 47. Lingulina costata, No. 5612. 48. Lingulina dentaliniformis, No. 5610. 49. Lingulina esseyana, No. 5611. 50. Lingulina laevissima, No. 5609. 51. Eoguttulina liassica, 547B-20-2, 59-61 cm, No. 5621 a. 52. Eoguttulina ventricosa, 547B-17,CC, No. 5623. 53. Eoguttulina metensis, No. 5622. 54. Eoguttulina oolithica, 547B-20-2, 55-57 cm, No. 5621 b.

Plate 2. Middle and Upper Jurassic foraminifers. (Magnification $\times 38$ unless otherwise indicated.) 55. Tolypammina jurensis, on test of a Lenticulina, 547B-7,CC, No. 5804. 56. Trochammina pulchra, 547B-7,CC, No. 5817, $\times 50$. 57-58. Bigenerina arcuata, (57) 547B-7-4, 135-137 cm, No. 5820, (58) 547B-7-2, 35-36 cm, No. 5821, ×50. 59. Bigenerina jurassica, 547B-7,CC, No. 5819. 60-61. Verneuilinoides tryphera, (60) 547B-8-1, 4-6 cm, No. 5824, $\times$ 50. (61) 547B-7-4, 135-137 cm, No. 5823, apex compressed. 62. Ophthalmidium sp., 547B-7,CC, No. 5876. 63-64. Triloculina meotica, 547B-7-4, 135-137, (63) No. 5829, (64) No. 5828. 65. Dentalina crenata, 547B-10-3, 125-128 cm, No. 5835. 66. Dentalina jurensis, 547B-10-3, 125-128 cm, No. 5837, $\times 75$. 67. Dentalina plebeia, 547B-10-3, 125-128 cm, No. 5836, $\times 75$. 68. Dentalina aff. pseudoarcuata, 547B-7,CC, No. 5839. 69. Dentalina turgida, 547B-10-3, $125-128 \mathrm{~cm}$, No. 5838.70 . Dentalina sp., 547B-7-4, 135-137 cm, No. 5842 . 71. Dentalina sp. A., 547B-6-4, $49-51 \mathrm{~cm}$, No. 5843. 72. Astacolus spongiphilus, $547 \mathrm{~B}-7-4,135-137 \mathrm{~cm}$, No. 5848.73 . Astacolus carinocostata, $547 \mathrm{~B}-10-3,125-128 \mathrm{~cm}$, No. 5844 . 74. Astacolus turgidus, $547 \mathrm{~B}-7, \mathrm{CC}$, No. $5847, \times 25$. 75 . Astacolus $\mathrm{cf}$. turgidus, 547B-10-3, 125-128 cm, No. 5845. 76. Citharina colliezi, 547B-10-3, 125-128 cm, No. $5849, \times 25$. 77. Citharina inconstans, 547B-10-3, $125-128 \mathrm{~cm}$, No. $5868, \times 25$. 78. Marginulina oolithica, 547B-10-3, 125-128 cm, No. 5856. 79. Palmula gyrata, 547B-7-4, 135-137 cm, No. $5863, \times 25$. 80. Palmula hybrida, 547B-10-3, $125-128 \mathrm{~cm}$, No. $5859, \times 25$. 81. Palmula anceps, 547B-7-2, $35-36 \mathrm{~cm}$, No. 5862 . 82 . Palmula primordialis, 547B-10-3, $125-128 \mathrm{~cm}$, No. $5860, \times 50$. 83. Pseudonodosaria dubia, $547 \mathrm{~B}-10-3,125-128 \mathrm{~cm}$, No. 5865 . 84. Pseudonodosaria turbiniformis, 547B-10-3, 125-128 cm, No. 5864. 85. Vaginulina implicata, 547B-10-3, 125-128 cm, No. 5864 . 86. Vaginulina aff. jurassi$c a, 547 \mathrm{~B}-7, \mathrm{CC}$, No. $5857, \times 25$. 87. Vaginulina sp., 547B-7,CC, No. $5850, \times 50$. 88. Eoguttulina bilocularis, 547B-10-3, 125-128 cm, No. 5877. 89. Eoguttulina laevis, 547B-7,CC, No. 5879. 90. Eoguttulina metensis, 547B-8-1, 4-6 cm, No. 5878. 91. Spirillina concava, 547B-10-3, 125-128 cm, No. 5883. 92. Spirillina gracilis, 547B-7-2, 35-36 cm, No. 5882. 93-94. Epistomina sp., 547B-7-4, 135-137 cm (93) No. 5887, (94) umbilical side, No. 5886, $\times 25$. 95. Epistomina sp. A, 547B-7-4, 135-137 cm, No. 5885 . 


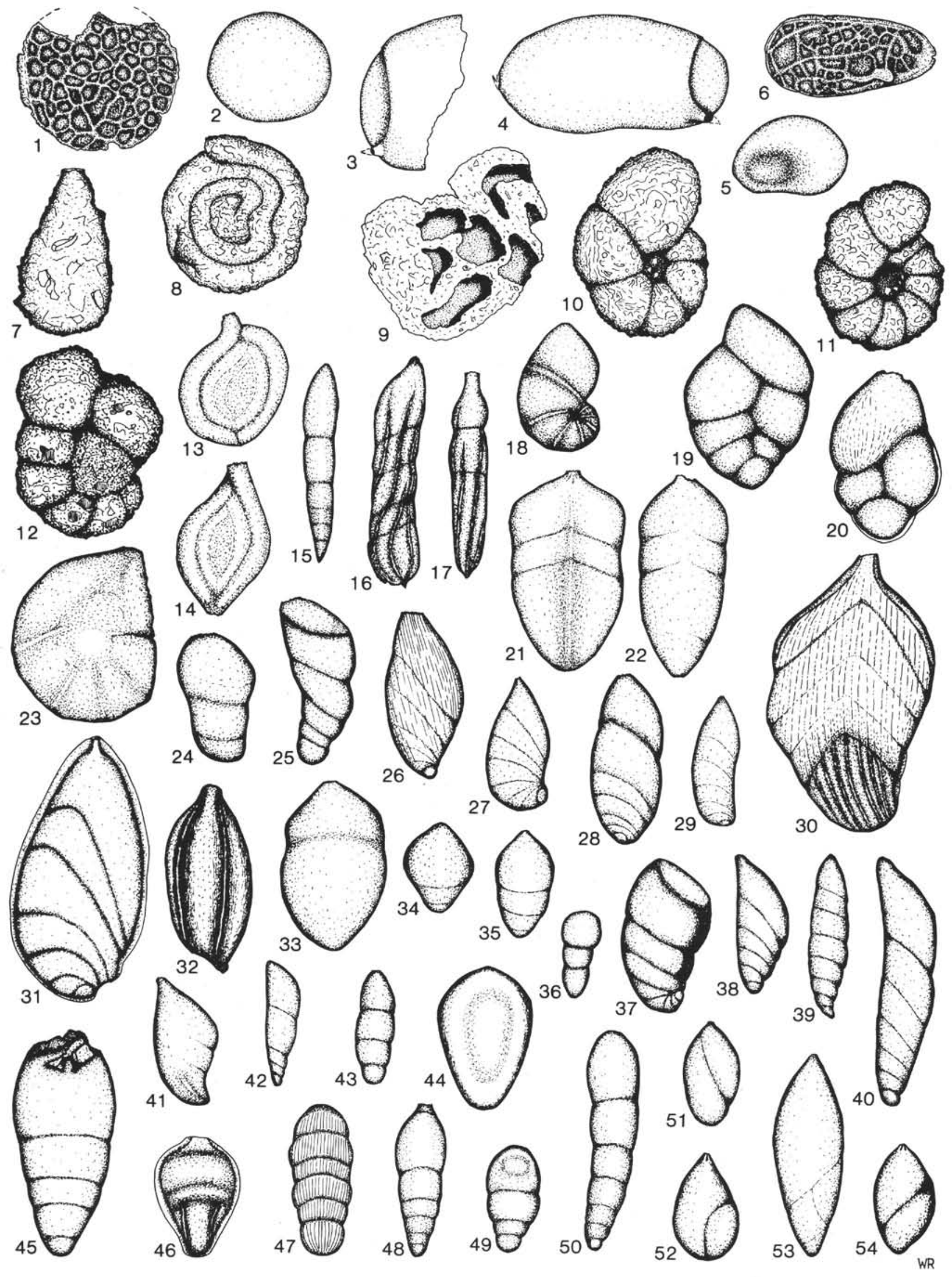



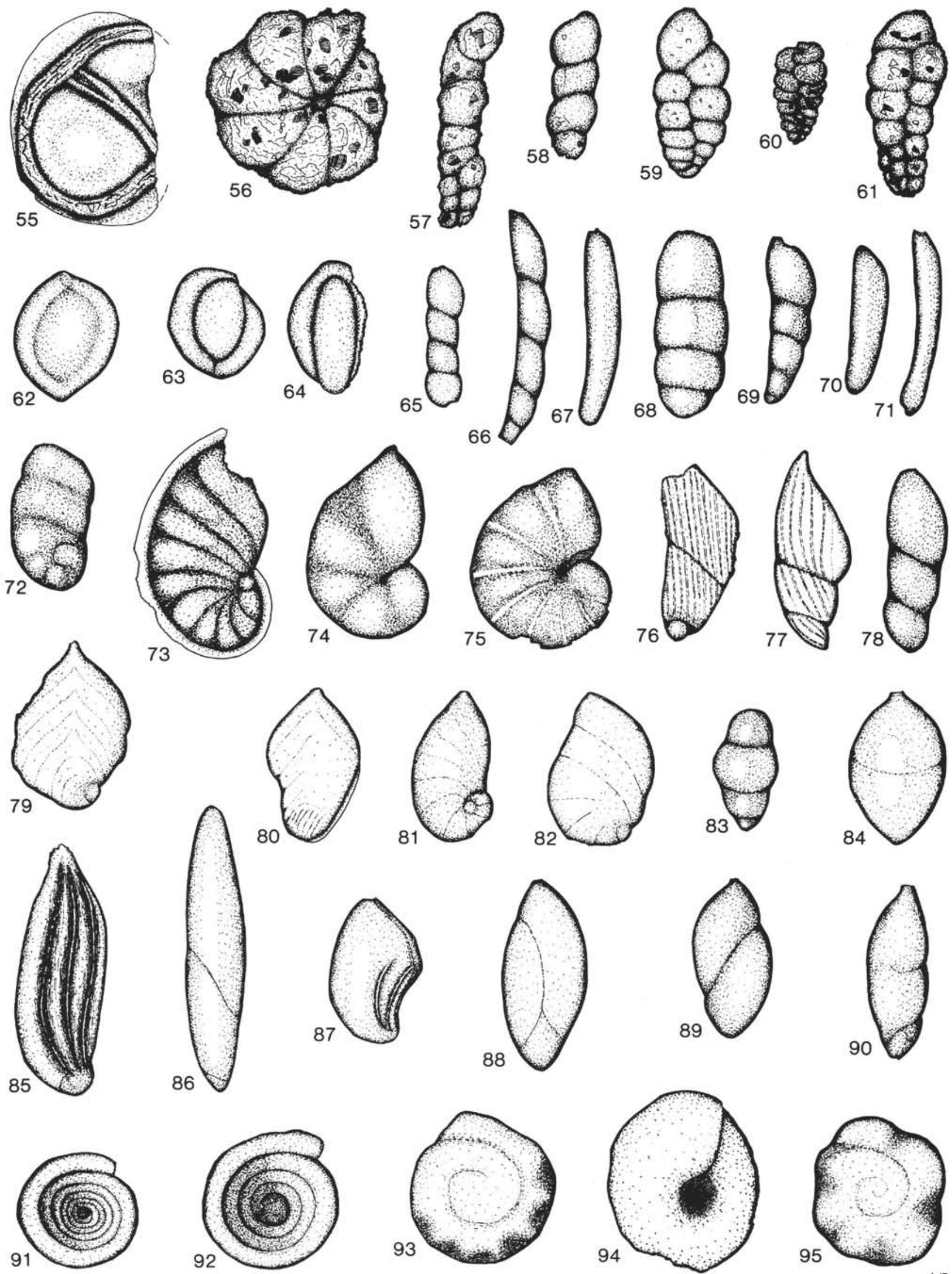


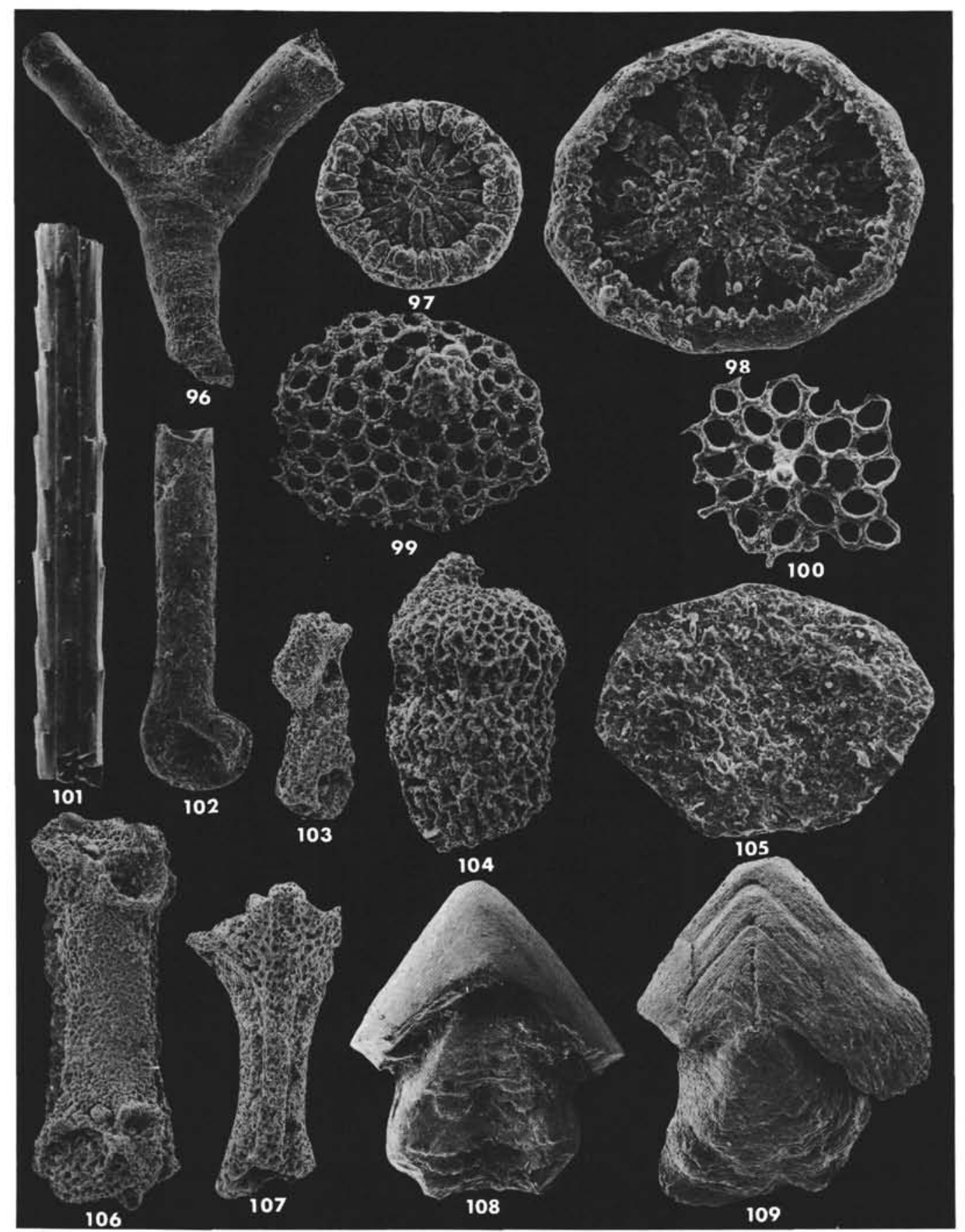

Plate 3. Echinodermata remains, bryozoans, cephalopod jaws. 96. Bryozoan indet., 547B-18-1, 22-24 cm, No. 5512, $\times 118.97$. Hemisphaeranthos cf. carpenteri, 547B-7-2, 35-36 cm, No. 5803, $\times 158$. 98. Theelia mesoliassica, 547B-18-2, 5-7 cm, No. 5506, $\times 316.99-100$. Eocaudina mortenseni, $\times 237$, (99) 547B-18-2, 5-7 cm, No. 5627, (100) 547B-20-1, 126-129 cm, No. 5628. 101. Diademopsis spine, 547B-20-2, $59-61 \mathrm{~cm}$, No. $5511, \times 79$. 102. Achistrum issleri, 547B-22-2, 55-57 cm, $\times 158.103$. Ophiomusium vertebra, 547B-22-2, 55-57 cm, No. $5510, \times 79$. 104. Echinoderm remains, 547B-8-1, 4-6 cm, No. 5858, $\times 158$. 105. Mortensenites?, 547B-22-2, 55-57 cm, No. 5626, $\times 237$. 106-107. Ophiomusium vertebra, 547B-22-2, 55-57 cm, $\times 79$, (106) No. 5508, (107) No. 5509. 108. Hadrocheilus liasinus (Zittel), 547B-18-2, 5-7 cm, No. 6034, $\times 70$. 109. Akidocheilus transiens Till, 547B-7-2, $35-36 \mathrm{~cm}, \times 32$. 


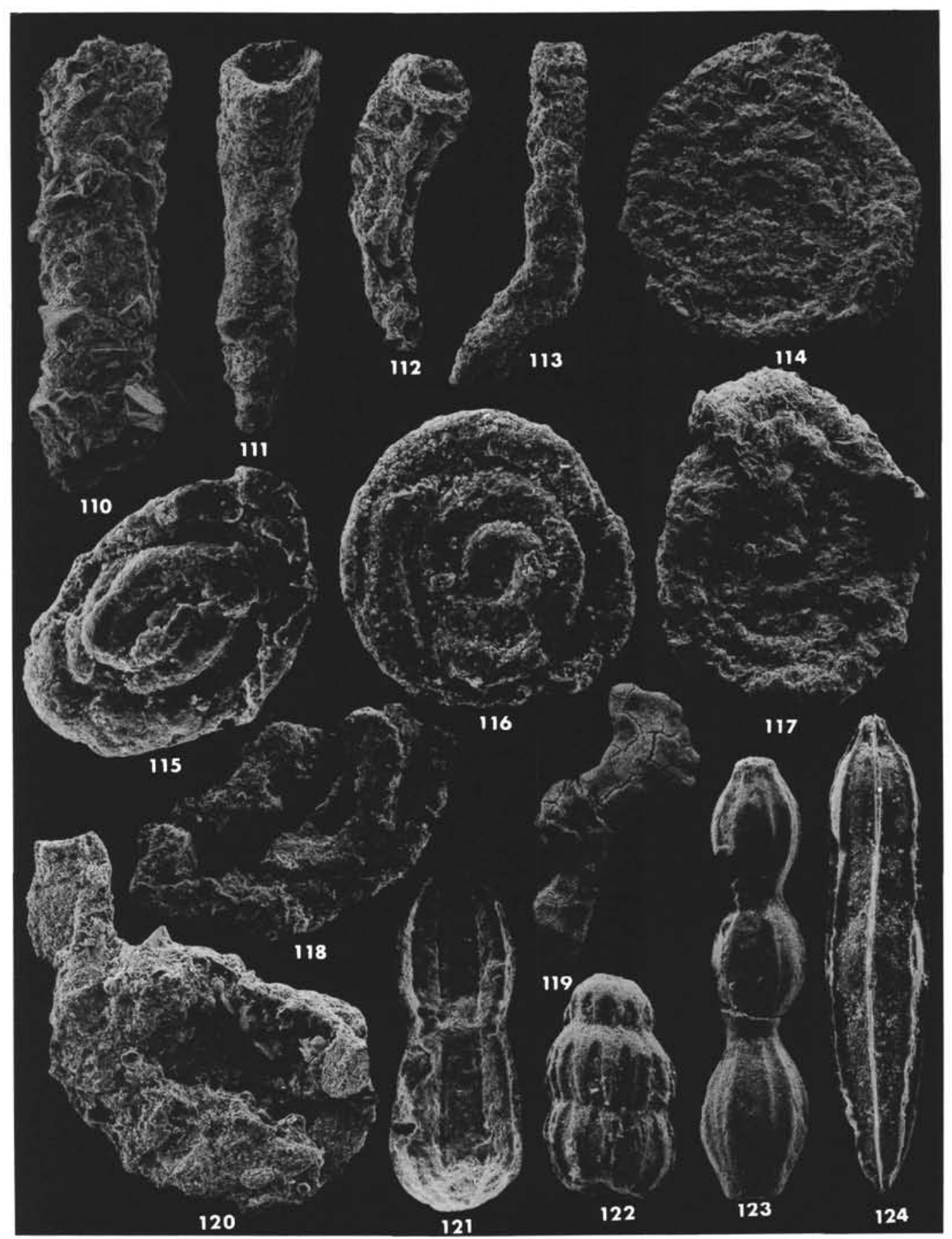

Plate 4. Liassic foraminifers: agglutinating species, miliolids, Nodosaria. 110. Hyperammina? liasina, 547B-20-2, 59-61 cm, No. 5513, $\times 79$. 111-112. Jaculella anulata Barbieri, 547B-20-2, 59-61 cm, $\times 118$, (111) No. 5514, (112) No. 5515 . 113. Hyperammina? sp., 547B-20-2, 59-61 cm, No. $5522, \times 118$. 114. Ammodiscus siliceus asper, 547B-22-2, 55-57 cm, No. 5517, $\times 79$. 115-116. Glomospira pattoni Tappan, 547B-22-2, $55-57 \mathrm{~cm}, \times 237,(115)$ No. 5520, (116) No. 5521. 117. Ammodiscus siliceus asper, 547B-20-2, 59-61 cm, No. 5518, $\times 79$. 118. ?Tolypammina sp., 547B-22-2, 55-57 cm, No. 5523. $\times 158$. 119. Ophthalmidium baccularis Issler, 547B-22-2, 55-57 cm, No. 5527, $\times 79$; cracks caused by preparation. 120 . ?Tolypammina sp., $547 \mathrm{~B}-22-2,55-57 \mathrm{~cm}$, No. $5530, \times 118$. 121. Nodosaria geniculata, $547 \mathrm{~B}-11, \mathrm{CC}$, No. $5535, \times 158$. 122. Nodosaria ornata, $547 \mathrm{~B}-20-1,126-129 \mathrm{~cm}$, No. $5538, \times 158$. 123. Nodosaria perlata, $547 \mathrm{~B}-18-2,5-7 \mathrm{~cm}$, No. $5539, \times 158$. 124. Nodosaria sexcostata, $547 \mathrm{~B}-18-2,5-7 \mathrm{~cm}$, No. $5541, \times 158$. 


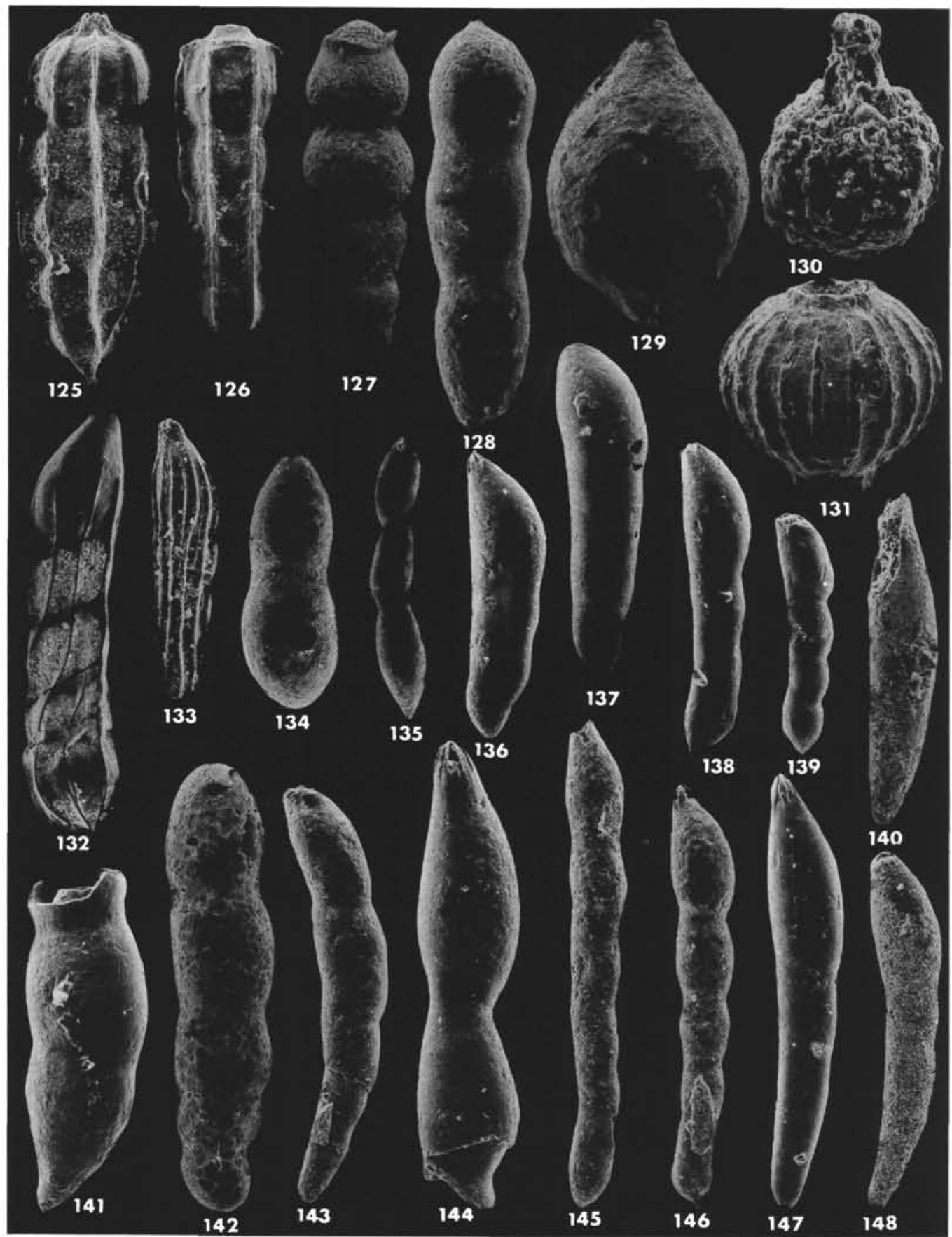

Plate 5. Liassic foraminifers: Nodosaria, Dentalina. (Magnification $\times 118$ unless otherwise indicated.) 125. Nodosaria alemannica, 547B-11,CC, No. $5598, \times 158$. 126. Nodosaria lamellosa, $547 \mathrm{~B}-20-1,126-129 \mathrm{~cm}$, No. $5537, \times 158 . \quad 127$. Nodosaria claviformis, $547 \mathrm{~B}-22-1,95-98 \mathrm{~cm}$, No. $5536, \times 158$. 128. Nodosaria simplex, $547 \mathrm{~B}-22-2,55-57 \mathrm{~cm}$, No. $5542, \times 158$. 129. Nodosaria regularis, 547B-11,CC, No. 55540 , $\times 158$. 130. Nodosaria apheilolocula, 547B-18-2, 5-7 cm, No. 5533, $\times 316$; spines recrystallized. 131. Nodosaria multicostata, 547B-11,CC, No. $5543, \times 158$. 132. Dentalina teutoburgensis, 547B-20-1, 126-129 cm, No. 5559. 133. Dentalina sculpta, 547B-22-2, 55-57 cm, No. 5557. 134. Dentalina biloculina, 547B-22-2, 55-57 cm, No. 5554 . 135. Dentalina nodigera, 547B-20-1, 126-129 cm, No. 5547 . 136. Dentalina integra, 547B-20-1, 126-129 cm, No. 5578. 137. Dentalina integra, 547B-20-1, 126-129 cm, No. 5555. 138. Dentalina subsiliqua, 547B-20-1, 126-129 cm, No. 5550. 139. Dentalina suboligostegia, 547B-20-1, 126-129 cm, No. 5561. 140. Dentalina cf. terquemi, 547B-18-1, $22-24 \mathrm{~cm}$, No. 5558. 141. Dentalina glandulinoides, 547B-20-1, 126-129 cm, No. 5544 . 142. Dentalina digitalis, 547B-11,CC, No. 5553. 143. Dentalina torta, 547B-22-2, 55-57 cm, No. 5546. 144-146. Dentalina vetustissima, (144) 547B-20-2, 59-61 cm, No. 5549, (145-146) 547B-22-2, 55-57 cm, Nos. 5562 and 5552. 147-148. Dentalina subulata, (147) 547B-20-1, 126-129 cm, No. 5551, (148) 547B-18-2, 5-7 cm, No. 5560 . 


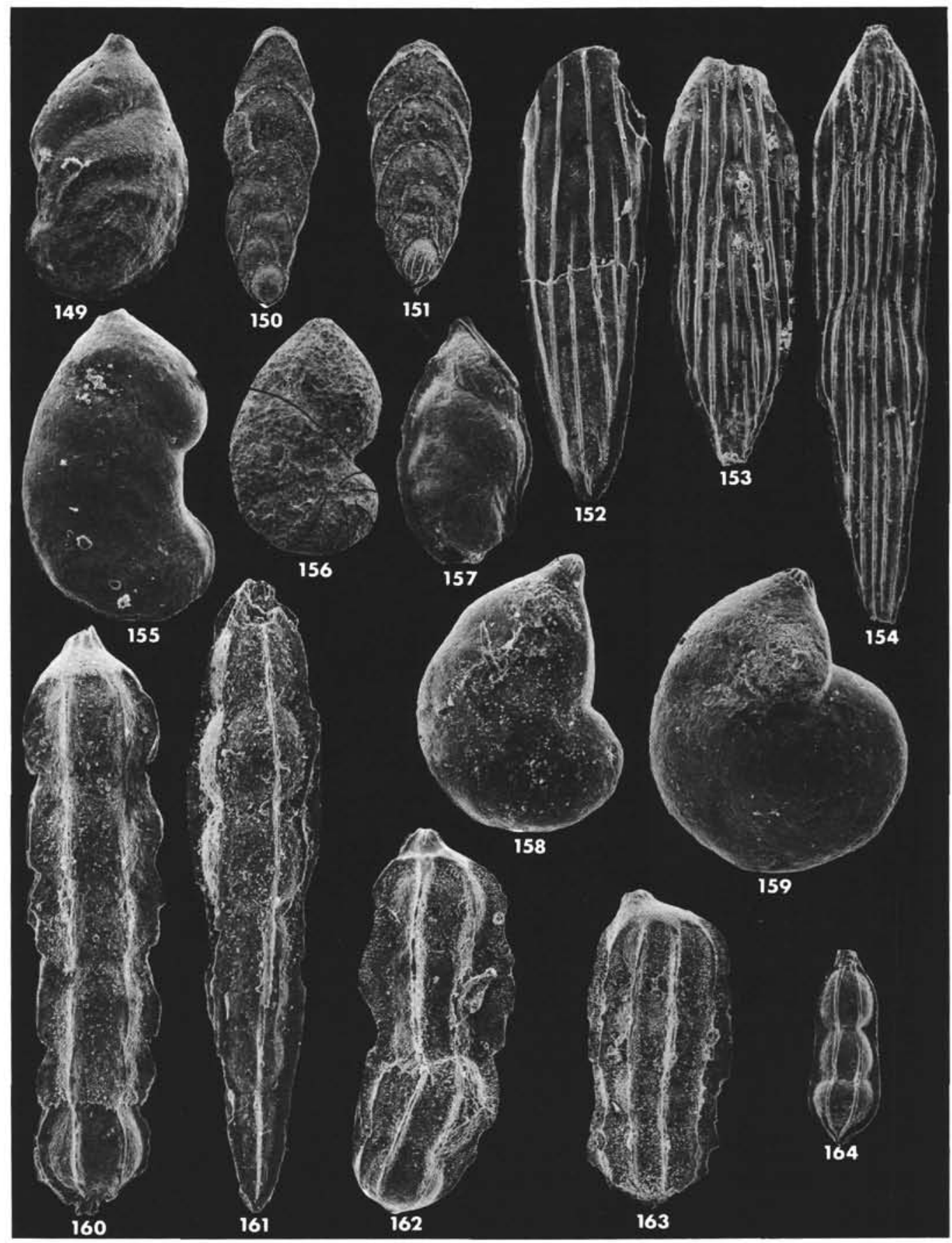

Plate 6. Liassic foraminifers: Astacolus, Frondicularia, Lenticulina, and Marginulina. (Magnification $\times 118$ unless otherwise indicated.) 149. Astacolus varians, 547B-20-1, 126-129 cm, No. 5567. 150-151. Frondicularia paradoxa Berthelin, 547B-22-2, 55-57 cm, (150) No. 5571, (151) No. 5570. 152. Frondicularia terquemi terquemi, 547B-20-2, 59-61 cm, No. 5574. 153. Frondicularia terquemi dubia, 547B-20-2, 59-61 cm, No. 5573. 154. Frondicularia squamosa, 547B-20-1, 126-129 cm, No. 5572. 155. Astacolus deformis, 547B-20-1, 126-129 cm, No. 5589. 156. Astacolus semiincisus, 547B-11,CC, No. 5565. 157. Astacolus stillus, 547B-20-1, 126-129 cm, No. 5564 . 158. Lenticulina inermis, 547B-20-2, 59-61 cm, No. 5576. 159. Lenticulina gottingensis, 547B-20-1, 126-129 cm, No. 5575. 160. Marginulina spinata, 547B-20-1, 126-129 cm, No. 5582. 161. Marginulina porrecta, 547B-22-2, 55-57 cm, No. 5581. 162. Marginulina prima, 547B-20-1, 126-129 cm, No. 5584. 163. Marginulina prima, 547B-20-1, 126-129 cm, No. 5583. 164. Marginulina variabilis, 547B-20-1, $126-129 \mathrm{~cm}$, No. 5586 a. 


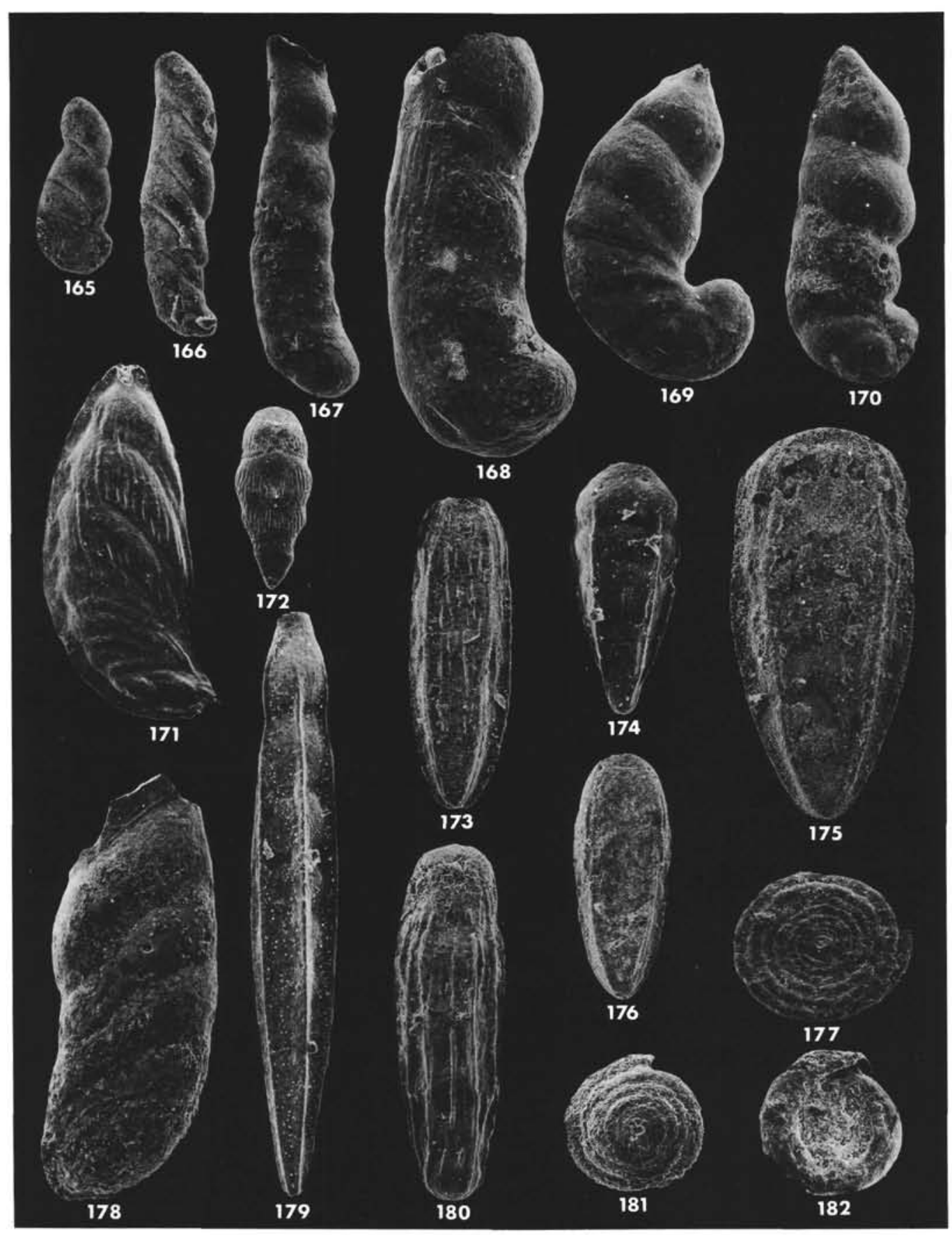

Plate 7. Liassic foraminifers: Marginulinopsis, Palmula, Lingulina, Vaginulina, Spirillina, and Trocholina. (Magnification $\times 118$, unless otherwise indicated.) 165. Marginulinopsis baccularis, 547B-22-1, $95-98 \mathrm{~cm}$, No. 5586. 166. Marginulinopsis pauliniae, 547B-18-2, 5-7 cm, No. 5580. 167-168. Marginulinopsis matutina matutina, 547B-20-1, 126-129 cm, (167) No. $5591 \mathrm{a},(168)$ No. 5588 . 169. Marginulinopsis matutina terquemi, 547B-20-1, 126-129 cm, No. 5587. 170. Marginulinopsis matutina vetusta, 547B-20-1, 126-129 cm, No. 5590. 171 . Palmula pikettyi, 547B-20-1, 126-129 cm, No. 5595. 172. Lingulina costata, 547B-20-1, 126-129 cm, No. 5606. 173. Lingulina tenera praepupa, 547B-22-2, 55-57 cm, No. 5617. 174-175. Lingulina tenera tenera, (174) 547B-20-2, 59-61 cm, No. 5619 b, (175) 547B-11,CC, No. 5618. 176. Lingulina tenera tenuistriata, 547B-18-1, 22-24 cm, No. 5619 a. 177. Spirillina numismalis, 547B-18-2, 5-7 cm, No. 5624 . 178 . Vaginulina contracta, 547B-20-1, 126-129 cm, No. 5563. 179. Lingulina acuformis, 547B-20-2, 59-61 cm, No. 5605 . 180. Lingulina tenera carinata, 547B-20-1, 126-129 cm, No. 5616. 181-182. Trocholina umbo, 547B-18-2, 5-7 cm, (181) No. 5625; dorsal. (182) No. 5625; ventral (umbilical side). 


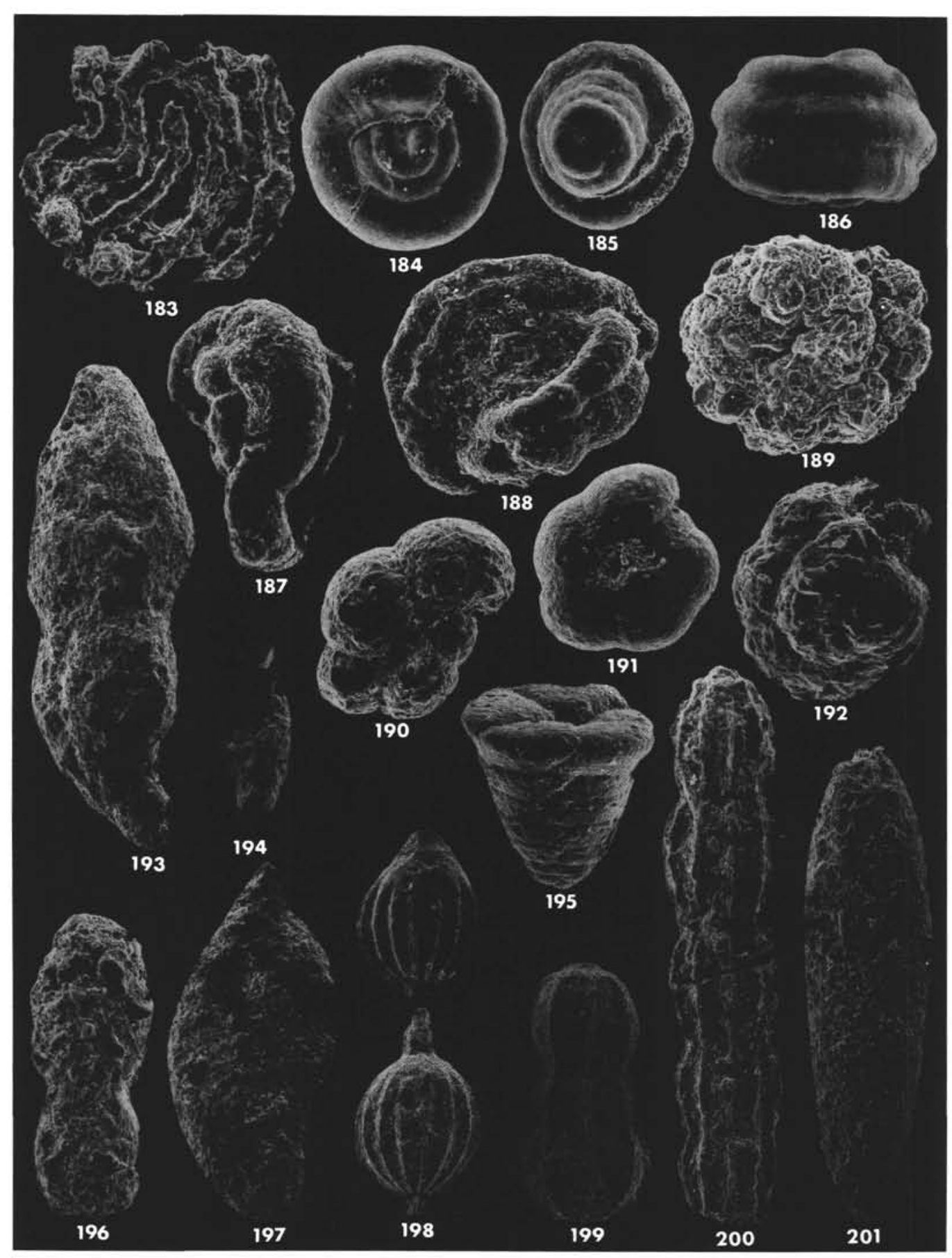

Plate 8. Middle and Upper Jurassic foraminifers: Agglutinating species, miliolids, Nodosaria. (Magnification $\times 158$ unless otherwise indicated.) 183. Ammovertella? plicata, 547B-7-4, 135-137 cm, No. 5805. 184-186. Glomospira variabilis, 547B-8-1, 4-6 cm, No. 5807, (185) Glomospira variabilis, No. 5808, (186) No. 5806. 187-188. Glomospira virgula 547B-8-1, 4-6 cm, (187) No. 5809, (188) No. 5810. 189. Trochammina canningensis, 547B-8-1, 4-6 cm, No. 5815. 190. Haplophragmoides globigerinoides, 547B-7-2, 35-36 cm, No. 5811. 191. Haplophragmoides hyalinus, 547B-7,CC, No. 5812. 192. Trochammina canningensis, 547B-7,CC, No. 5818. 193. Reophax imperspicuus, 547B-7-4, 135-137 cm, No. $5814, \times 79$. 194. Ophthalmidium strumosum, $547 \mathrm{~B}-7-4,135-137 \mathrm{~cm}$, No. $5827, \times 118 . \quad 195$. Dorothia dumortieri, 547B-7-2, $35-36 \mathrm{~cm}$, No. 5822. 196. Reophax helveticus, 547B-7-4, 135-137 cm, No. $5813, \times 118$. 197. Ophthalmidium strumosum, 547B-7-4, 135-137 cm, No. $5825, \times 118$. 198. Nodosaria lagenoides, 547B-7,CC, No. 5832; two fragments, $\times 79$. 199. Nodosaria incongrua, 547B-7,CC, No. 5833, $\times$ 118. 200. Nodosaria kunzi, 547B-10-3, $125-128 \mathrm{~cm}$, No. $5830, \times 118.201$. Nodosaria clavula, $547 \mathrm{~B}-10-3,125-128 \mathrm{~cm}$, No. $5831, \times 118$. 


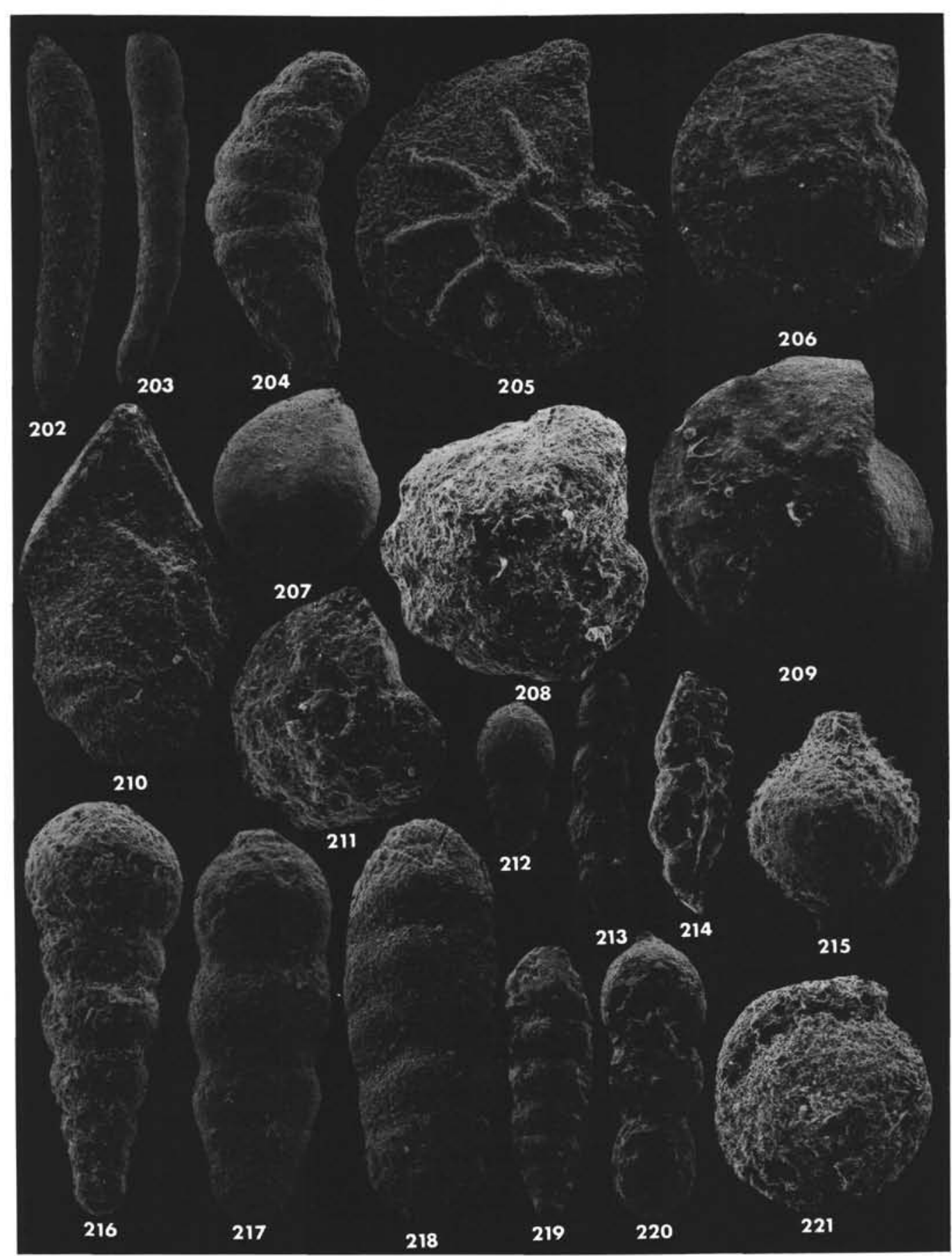

Plate 9. Middle and Upper Jurassic foraminifers: Dentalina, Lenticulina, Palmula, Lingulina, Eoguttulina, Ramulina, and Epistomina. (Magnification $\times 118$ unless otherwise indicated.) 202. Dentalina cushmani, 547B-7,CC, No. 5841. $\times 79$. 203. Dentalina haeusleri, 547B-7,CC, No. 5840 204. Dentalina alternans, $547 \mathrm{~B}-10-3,125-128 \mathrm{~cm}$, No. 5834 . 205. Lenticulina quenstedti, $547 \mathrm{~B}-8-1,4-6 \mathrm{~cm}$, No. 5853 . 206 . Lenticu5840. 204. Dentalina alternans, $547 \mathrm{~B}-10-3,125-128 \mathrm{~cm}$, No. 5834 . 205ticulina hebetata, $547 \mathrm{~B}-7, \mathrm{CC}, \mathrm{No} .5855 . \times 40$. 208. Lenticulina? pusilla, $547 \mathrm{~B}-7-4,135-137 \mathrm{~cm}$, No. 5881. 209. Lenticulina hebetata, 547B-7,CC, No. 5843. $\times 40$. 210. Palmula triquetra, 547B-7, CC, No. 5861. 211. Lenticulina oolithica, 547B-10-3, 125-128 cm, No. 5851. 212. Lingulina sp., 547B-8-1, 4-6 cm, No. 5871. 213. Lingulina nodosaria, 547B-10-3, 125-128 cm, No. 5870. 214. Eoguttulina subrhomboidalis, 547B-7-2, 35-36 cm, No. 5866. 215. Ramulina cf. spandeli, 547B-8-1,

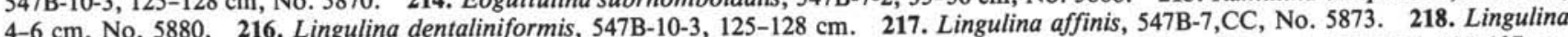
franconica, 547B-7,CC, No. 5875. 219. Lingulina franconica, 547B-7, CC, No. 5872. 220. Lingulina cf. dentaliniformis, 547B-7-4, 135-137 cm, No. 5874. 221. Epistomina? sp., 547B-7,CC. No. 5884. 


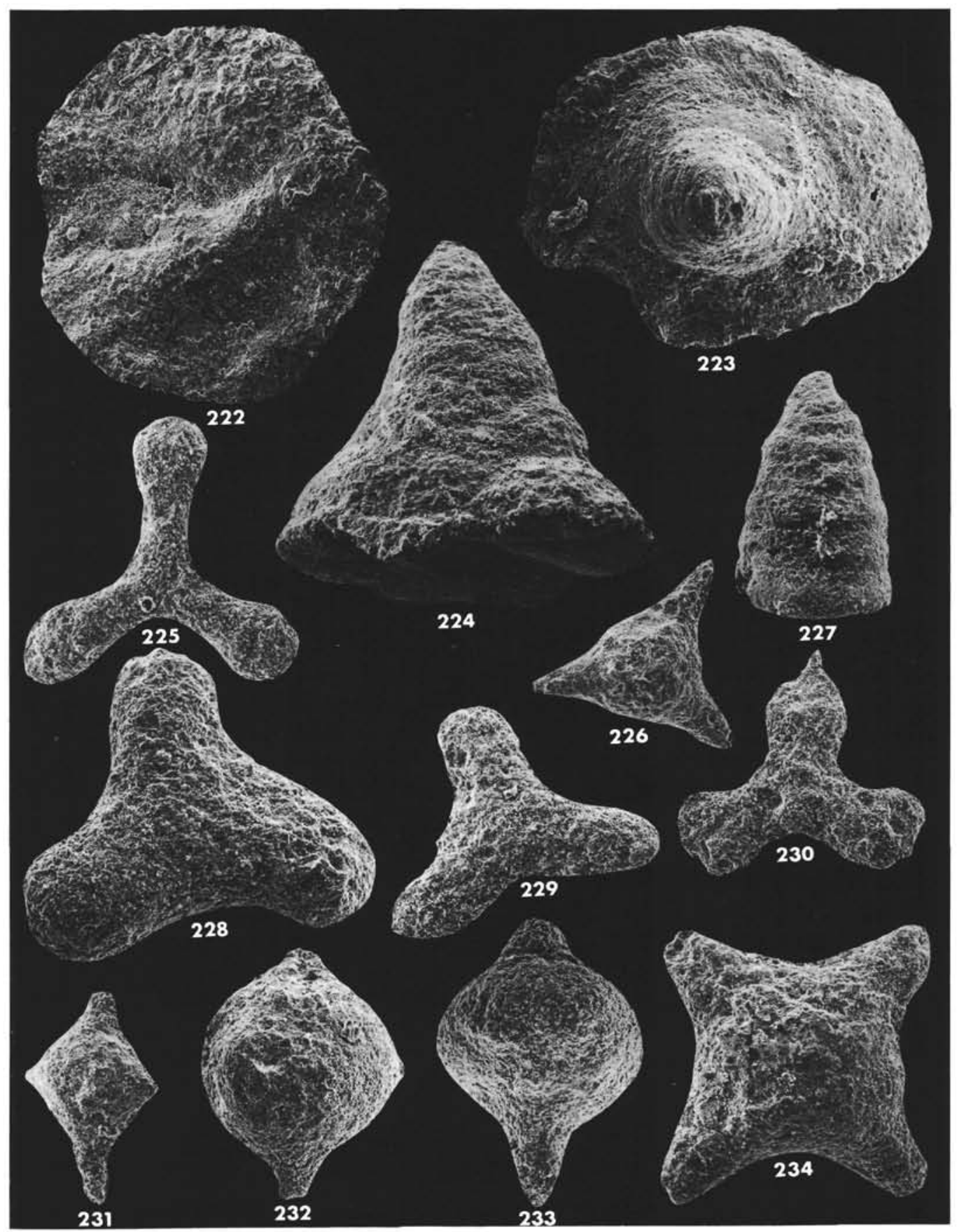

Plate 10. Upper Jurassic foraminifers: "Patellinella"; Middle and Upper Jurassic radiolarians. (Magnification $\times 118$ unless otherwise indicated.) 222-224. "Patellinella" poddari, 547B-7-4, 135-137 cm, $\times 79,(222)$ No. 5891; ventral (umbilical side), (223) No. 5890; dorsal, (224) No. 5889; lateral. 225. Paronaella sp., 547B-7-4, 135-137 cm, No. 5894. 226. Triactoma cf. blakei (Pessagno), 547B-7-4, 135-137 cm, No. 5896.227. Dictyomitra cf. duodecimcostata (Squinabol), 547B-10-3, 125-128 cm, No. 5893. 228-229. Paronaella? sp., (228) 547B-7-4, 135-137 cm, No. 5895, (229) 547B-10-3, 125-128 cm, No. 5892. 230. Triactinosphaera? sp., 547B-7-4, 135-137 cm, No. 5895. 231. Podobursa cf. pantanellii (Parona), 547B-7-4, 135-137 cm, No. 5900. 232-233. Gen. indet., 547B-7-4, 135-137 cm, (232) No. 5899, (233) No. 5897. 234. Emiluvia sp., 547B-7-4, 135-137 cm., No. 5898. 